\title{
Routine laboratory testing to determine if a patient has COVID-19
} (Review)

Stegeman I, Ochodo EA, Guleid F, Holtman GA, Yang B, Davenport C, Deeks JJ, Dinnes J, Dittrich S, Emperador D, Hooft L, Spijker R, Takwoingi Y, Van den Bruel A, Wang J, Langendam M, Verbakel JY, Leeflang MMG, Cochrane COVID-19 Diagnostic Test Accuracy Group

Stegeman I, Ochodo EA, Guleid F, Holtman GA., Yang B, Davenport C, Deeks JJ, Dinnes J, Dittrich S, Emperador D, Hooft L, Spijker R, Takwoingi Y, Van den Bruel A, Wang J, Langendam M, Verbakel JY, Leeflang MMG.

Routine laboratory testing to determine if a patient has COVID-19.

Cochrane Database of Systematic Reviews 2020, Issue 11. Art. No.: CD013787.

DOI: 10.1002/14651858.CD013787.

www.cochranelibrary.com 
TABLE OF CONTENTS

HEADER

ABSTRACT

PLAIN LANGUAGE SUMMARY ....

SUMMARY OF FINDINGS

BACKGROUND

OBJECTIVES

METHODS

RESULTS

Figure 1.

Figure 2.

Figure 3.

Figure 4.

Figure 5.

Figure 6.

Figure 7.

Figure 8.

Figure 9.

Figure 10.

Figure 11.

Figure 12.

Figure 13.

Figure 14.

DISCUSSION

AUTHORS' CONCLUSIONS

ACKNOWLEDGEMENTS

REFERENCES

CHARACTERISTICS OF STUDIES

DATA

Test 1. WBC increase

Test 2. WBC decrease

Test 3. Leukocyturia

Test 4. Monocyte count increase

Test 5. Monocyte count decrease

Test 6. Monocyte percentage increase

Test 7. Neutrophil count increase

Test 8. Neutrophil count decrease

Test 9. Neutrophil percentage increase

Test 10. Neutrophil Percentage decrease

Test 11. Lymphocyte count increase

Test 12. Lymphocyte count decrease

Test 13. Lymphocyte percentage increase

Test 14. Lymphocyte percentage decrease

Test 15. Eosinophil count increase

Test 16. Eosinophil count decrease

Test 17. Eosinophil percentage increase

Test 18. Basophil count increase

Test 19. Basophil percentage increase

Test 20. Red Blood Cell volume distribution increase

Test 21. RBC decrease

Test 22. Platelets decreased

Test 23. Haemoglobin (HGB) Decreased 
Test 24. Serum creatinine increased $\quad$.........................................................................................................................

Test 25. Creatine Kinase - increase ...................................................................................................................................

Test 26. Creatine Kinase MB - increase ..................................................................................................................... 102

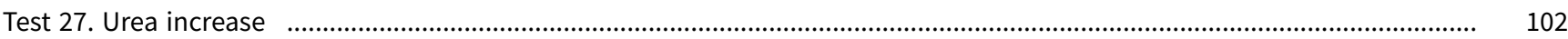

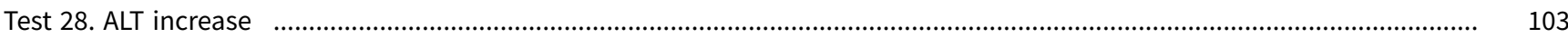

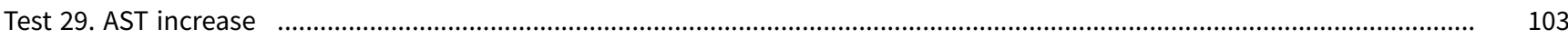

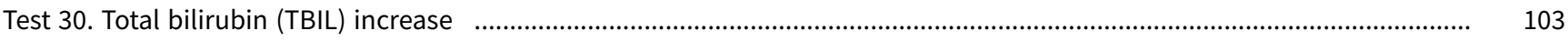

Test 31. Erythrocyte Sedimentation Rate (ESR) increase ............................................................................................

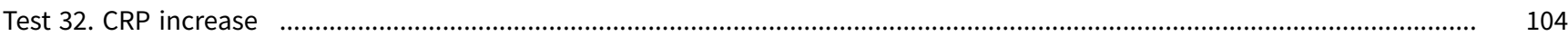

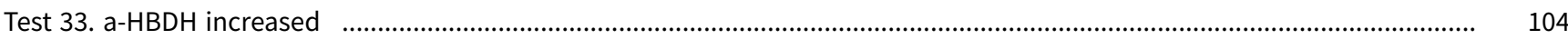

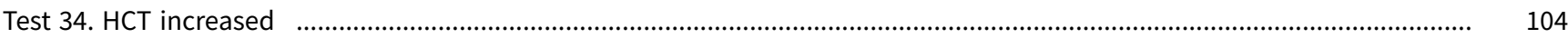

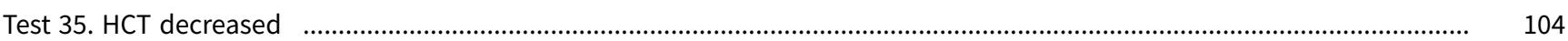

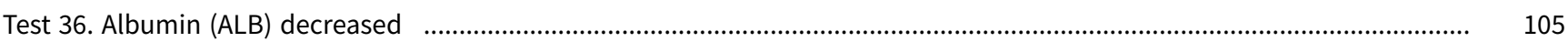

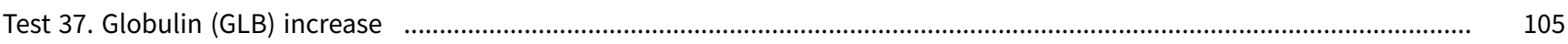

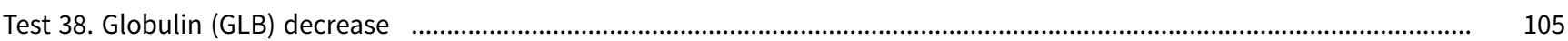

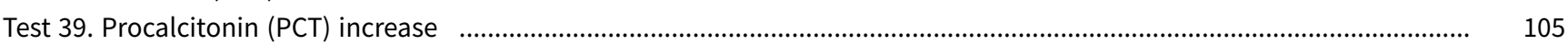

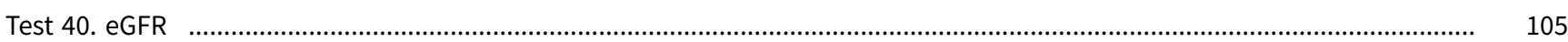

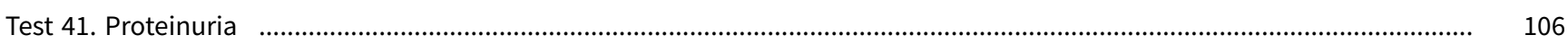

Test 42. Prothrombin time (PT) increase ...................................................................................................................... 106

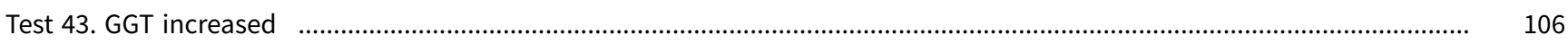

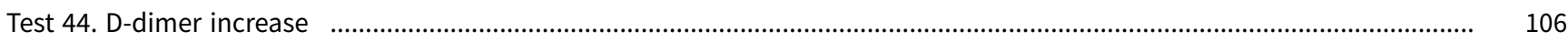

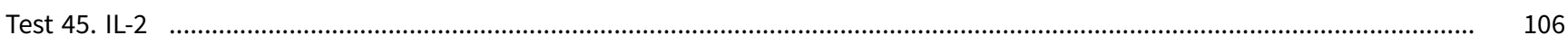

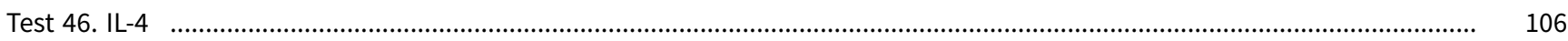

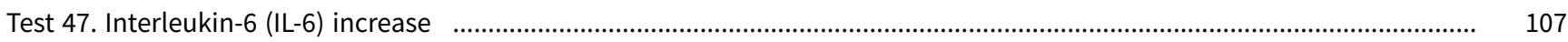

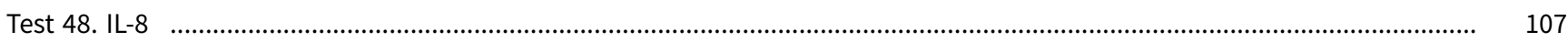

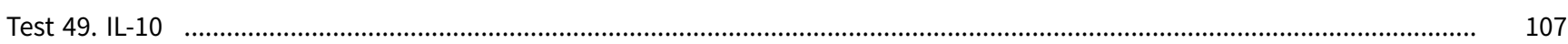

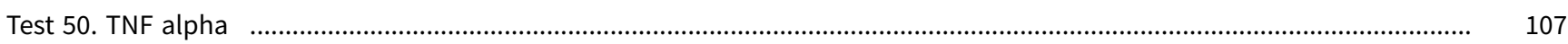

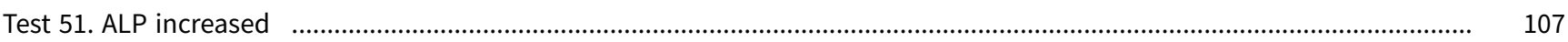

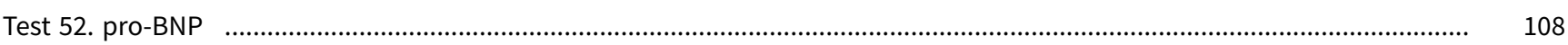

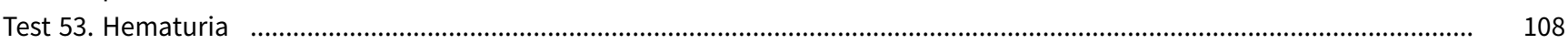

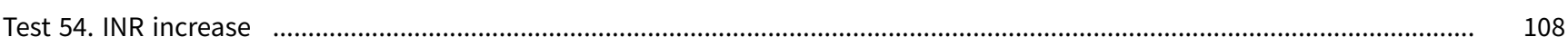

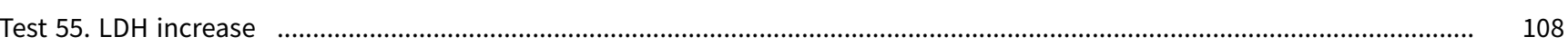

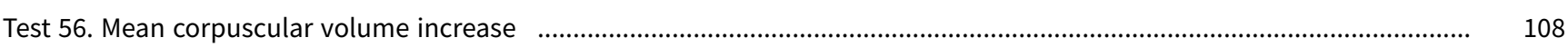

Test 57. Mean corpuscular volume decrease

Test 58. Erythrocyte mean corpuscular hemoglobin increase ................................................................................................ 109

Test 59. Erythrocyte mean corpuscular hemoglobin decrease ............................................................................................. 109

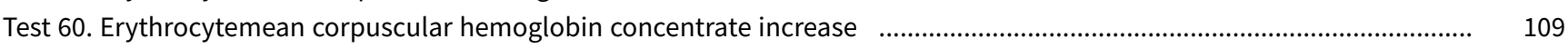

Test 61. Erythrocytemean corpuscular hemoglobin concentrate decrease ............................................................................. 109

Test 62. Mean Platelet Volume ........................................................................................................................................... 109

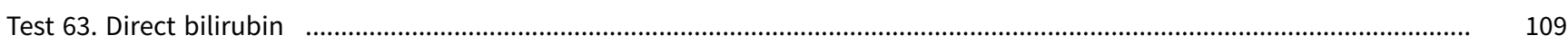

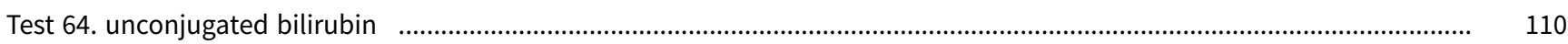

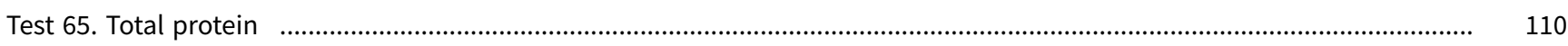

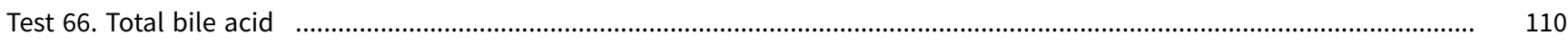

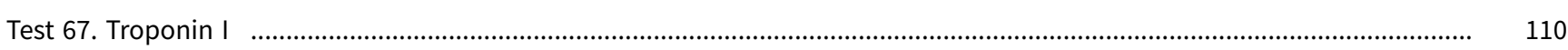

ADDITIONAL TABLES

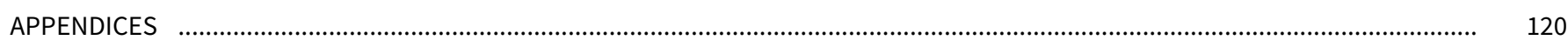

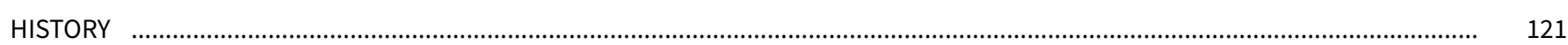

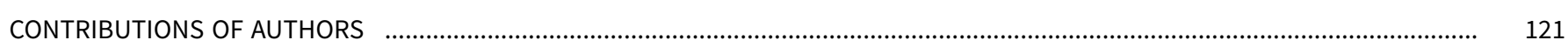

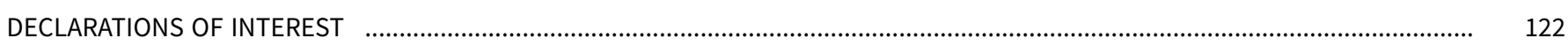

SOURCES OF SUPPORT

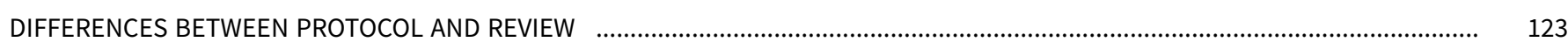

Routine laboratory testing to determine if a patient has COVID-19 (Review)

Copyright $\odot 2020$ The Authors. Cochrane Database of Systematic Reviews published by John Wiley \& Sons, Ltd. on behalf of The Cochrane

Collaboration. 


\section{Routine laboratory testing to determine if a patient has COVID-19}

Inge Stegeman1,2,3, Eleanor A Ochodo4,5, Fatuma Guleid6, Gea A. Holtman77, Bada Yang2 , Clare Davenport8,9, Jonathan J Deeks 8,9 , Jacqueline Dinnes8,9, Sabine Dittrich10, Devy Emperador10, Lotty Hooft11, René Spijker11,12, Yemisi Takwoingi8,9, Ann Van den Bruel13, Junfeng Wang14, Miranda Langendam², Jan Y Verbakel13, Mariska MG Leeflang², Cochrane COVID-19 Diagnostic Test Accuracy Group ${ }^{9}$

1Department of Otorhinolaryngology \& Head and Neck Surgery, University Medical Center Utrecht, Utrecht, Netherlands. ${ }^{2}$ Epidemiology and Data Science, Amsterdam University Medical Centers, University of Amsterdam, Amsterdam, Netherlands. ${ }^{3}$ Brain Center Rudolf Magnus, University Medical Center Utrecht, Utrecht, Netherlands. ${ }^{4}$ Centre for Evidence-based Health Care, Department of Global Health, Faculty of Medicine and Health Sciences, Stellenbosch University, Cape Town, South Africa. ${ }^{5}$ Centre for Global Health Research, Kenya Medical Research Institute, Kisumu, Kenya. ${ }^{6}$ KEMRI-Wellcome Trust Research Programme, Nairobi, Kenya. ${ }^{7}$ Department of General Practice, University of Groningen, University Medical Centre Groningen, Groningen, Netherlands. ${ }^{8}$ Test Evaluation Research Group, Institute of Applied Health Research, University of Birmingham, Birmingham, UK. ${ }^{9}$ NIHR Birmingham Biomedical Research Centre, University Hospitals Birmingham NHS Foundation Trust and University of Birmingham, Birmingham, UK. 10FIND, Geneva, Switzerland. ${ }^{11}$ Cochrane Netherlands, Julius Center for Health Sciences and Primary Care, University Medical Center Utrecht, Utrecht University, Utrecht, Netherlands. ${ }^{12}$ Medical Library, Amsterdam UMC, University of Amsterdam, Amsterdam Public Health, Amsterdam, Netherlands. 13Department of Public Health and Primary Care, KU Leuven, Leuven, Belgium. ${ }^{14}$ Julius Center for Health Sciences and Primary Care, University Medical Center Utrecht, Utrecht, Netherlands

Contact address: Mariska MG Leeflang, m.m.leeflang@amsterdamumc.nl, m.m.leeflang@amc.uva.nl.

Editorial group: Cochrane Infectious Diseases Group.

Publication status and date: New, published in Issue 11, 2020.

Citation: Stegeman I, Ochodo EA, Guleid F, Holtman GA., Yang B, Davenport C, Deeks JJ, Dinnes J, Dittrich S, Emperador D, Hooft L, Spijker R, Takwoingi Y, Van den Bruel A, Wang J, Langendam M, Verbakel JY, Leeflang MMG. Routine laboratory testing to determine if a patient has COVID-19. Cochrane Database of Systematic Reviews 2020, Issue 11. Art. No.: CD013787. DOI: 10.1002/14651858.CD013787.

Copyright (c) 2020 The Authors. Cochrane Database of Systematic Reviews published by John Wiley \& Sons, Ltd. on behalf of The Cochrane Collaboration. This is an open access article under the terms of the Creative Commons Attribution-Non-Commercial Licence, which permits use, distribution and reproduction in any medium, provided the original work is properly cited and is not used for commercial purposes.

\section{A B S T R A C T}

\section{Background}

Specific diagnostic tests to detect severe acute respiratory syndrome coronavirus 2 (SARS-CoV-2) and resulting COVID-19 disease are not always available and take time to obtain results. Routine laboratory markers such as white blood cell count, measures of anticoagulation, C-reactive protein (CRP) and procalcitonin, are used to assess the clinical status of a patient. These laboratory tests may be useful for the triage of people with potential COVID-19 to prioritize them for different levels of treatment, especially in situations where time and resources are limited.

\section{Objectives}

To assess the diagnostic accuracy of routine laboratory testing as a triage test to determine if a person has COVID-19.

\section{Search methods}

On 4 May 2020 we undertook electronic searches in the Cochrane COVID-19 Study Register and the COVID-19 Living Evidence Database from the University of Bern, which is updated daily with published articles from PubMed and Embase and with preprints from medRxiv and bioRxiv. In addition, we checked repositories of COVID-19 publications. We did not apply any language restrictions. 


\section{Selection criteria}

We included both case-control designs and consecutive series of patients that assessed the diagnostic accuracy of routine laboratory testing as a triage test to determine if a person has COVID-19. The reference standard could be reverse transcriptase polymerase chain reaction (RT-PCR) alone; RT-PCR plus clinical expertise or and imaging; repeated RT-PCR several days apart or from different samples; WHO and other case definitions; and any other reference standard used by the study authors.

\section{Data collection and analysis}

Two review authors independently extracted data from each included study. They also assessed the methodological quality of the studies, using QUADAS-2. We used the 'NLMIXED' procedure in SAS 9.4 for the hierarchical summary receiver operating characteristic (HSROC) metaanalyses of tests for which we included four or more studies. To facilitate interpretation of results, for each meta-analysis we estimated summary sensitivity at the points on the SROC curve that corresponded to the median and interquartile range boundaries of specificities in the included studies.

\section{Main results}

We included 21 studies in this review, including 14,126 COVID-19 patients and 56,585 non-COVID-19 patients in total. Studies evaluated a total of 67 different laboratory tests. Although we were interested in the diagnotic accuracy of routine tests for COVID-19, the included studies used detection of SARS-CoV-2 infection through RT-PCR as reference standard. There was considerable heterogeneity between tests, threshold values and the settings in which they were applied. For some tests a positive result was defined as a decrease compared to normal vaues, for other tests a positive result was defined as an increase, and for some tests both increase and decrease may have indicated test positivity. None of the studies had either low risk of bias on all domains or low concerns for applicability for all domains. Only three of the tests evaluated had a summary sensitivity and specificity over $50 \%$. These were: increase in interleukin-6, increase in Creactive protein and lymphocyte count decrease.

\section{Blood count}

Eleven studies evaluated a decrease in white blood cell count, with a median specificity of $93 \%$ and a summary sensitivity of $25 \%$ (95\% $\mathrm{Cl} 8.0 \%$ to 27\%; very low-certainty evidence). The 15 studies that evaluated an increase in white blood cell count had a lower median specificity and a lower corresponding sensitivity. Four studies evaluated a decrease in neutrophil count. Their median specificity was $93 \%$, corresponding to a summary sensitivity of $10 \%$ ( $95 \% \mathrm{Cl} 1.0 \%$ to $56 \%$; low-certainty evidence). The 11 studies that evaluated an increase in neutrophil count had a lower median specificity and a lower corresponding sensitivity. The summary sensitivity of an increase in neutrophil percentage (4 studies) was $59 \%$ ( $95 \% \mathrm{Cl} 1.0 \%$ to $100 \%$ ) at median specificity (38\%; very low-certainty evidence). The summary sensitivity of an increase in monocyte count (4 studies) was $13 \%(95 \% \mathrm{Cl} 6.0 \%$ to $26 \%)$ at median specificity (73\%; very low-certainty evidence). The summary sensitivity of a decrease in lymphocyte count (13 studies) was $64 \%$ (95\% Cl $28 \%$ to $89 \%$ ) at median specificity (53\%; low-certainty evidence). Four studies that evaluated a decrease in lymphocyte percentage showed a lower median specificity and lower corresponding sensitivity. The summary sensitivity of a decrease in platelets (4 studies) was $19 \%$ ( $95 \% \mathrm{Cl} 10 \%$ to $32 \%$ ) at median specificity ( $88 \%$; lowcertainty evidence).

\section{Liver function tests}

The summary sensitivity of an increase in alanine aminotransferase (9 studies) was $12 \%$ (95\% Cl 3\% to 34\%) at median specificity (92\%; low-certainty evidence). The summary sensitivity of an increase in aspartate aminotransferase (7 studies) was $29 \%$ (95\% Cl 17\% to $45 \%$ ) at median specificity (81\%) (low-certainty evidence). The summary sensitivity of a decrease in albumin (4 studies) was $21 \%$ ( $95 \% \mathrm{Cl} 3 \%$ to $67 \%$ ) at median specificity (66\%; low-certainty evidence). The summary sensitivity of an increase in total bilirubin (4 studies) was $12 \%$ (95\% $\mathrm{Cl} 3.0 \%$ to $34 \%)$ at median specificity (92\%; very low-certainty evidence).

\section{Markers of inflammation}

The summary sensitivity of an increase in CRP (14 studies) was $66 \%$ (95\% CI 55\% to 75\%) at median specificity (44\%; very low-certainty evidence). The summary sensitivity of an increase in procalcitonin (6 studies) was $3 \%(95 \% \mathrm{Cl} 1 \%$ to $19 \%)$ at median specificity ( $86 \%$; very low-certainty evidence). The summary sensitivity of an increase in IL-6 (four studies) was $73 \%$ (95\% Cl 36\% to 93\%) at median specificity (58\%) (very low-certainty evidence).

\section{Other biomarkers}

The summary sensitivity of an increase in creatine kinase (5 studies) was $11 \%$ (95\% Cl 6\% to 19\%) at median specificity ( $94 \%$ ) (low-certainty evidence). The summary sensitivity of an increase in serum creatinine (four studies) was $7 \%(95 \% \mathrm{Cl} 1 \%$ to $37 \%)$ at median specificity (91\%; low-certainty evidence). The summary sensitivity of an increase in lactate dehydrogenase (4 studies) was $25 \%$ (95\% Cl $15 \%$ to $38 \%$ ) at median specificity (72\%; very low-certainty evidence).

\section{Authors' conclusions}

Although these tests give an indication about the general health status of patients and some tests may be specific indicators for inflammatory processes, none of the tests we investigated are useful for accurately ruling in or ruling out COVID-19 on their own. Studies 
were done in specific hospitalized populations, and future studies should consider non-hospital settings to evaluate how these tests would perform in people with milder symptoms.

\section{PLAIN LANGUAGE SUMMARY}

\section{How accurate are routine laboratory tests for diagnosis of COVID-19?}

\section{What are routine laboratory tests?}

Routine laboratory tests are blood tests that assess the health status of a patient. Tests include counts of different types of white blood cells (these help the body fight infection), and detection of markers (proteins) that indicate organ damage, and general inflammation. These tests are widely available and in some places they may be the only tests available for diagnosis of COVID-19.

\section{What did we want to find out?}

People with suspected COVID-19 need to know quickly whether they are infected so that they can self-isolate, receive treatment, and inform close contacts.

Currently, the standard test for COVID-19 is usually the RT-PCR test. In the RT-PCR, samples from the nose and throat are sent away for testing, usually to a large, central laboratory with specialist equipment. Other tests include imaging tests, like X-rays, which also require specialist equipment.

We wanted to know whether routine laboratory tests were sufficiently accurate to diagnose COVID-19 in people with suspected COVID-19. We also wanted to know whether they were accurate enough to prioritize patients for different levels of treatment.

\section{What did we do?}

We searched for studies that assessed the accuracy of routine laboratory tests to diagnose COVID-19 compared with RT-PCR or other tests. Studies could be of any design and be set anywhere in the world. Studies could include participants of any age or sex, with suspected COVID-19, or use samples from people known to have - or not to have - COVID-19.

\section{What we found}

We found 21 studies that looked at 67 different routine laboratory tests for COVID-19. Most of the studies looked at how accurately these tests diagnosed infection with the virus causing COVID-19. Four studies included both children and adults, 16 included only adults and one study only children. Seventeen studies were done in China, and one each in Iran, Italy, Taiwan and the USA. All studies took place in hospitals, except one that used samples from a database. Most studies used RT-PCR to confirm COVID-19 diagnosis.

Accuracy of tests is most often reported using 'sensitivity' and 'specificity'. Sensitivity is the proportion of people with COVID-19 correctly detected by the test; specificity is the proportion of people without COVID-19 who are correctly identified by the test. The nearer sensitivity and specificity are to $100 \%$, the better the test. A test to prioritize people for treatment would require a high sensitivity of more than $80 \%$.

Where four or more studies evaluated a particular test, we pooled their results and analyzed them together. Our analyses showed that only three of the tests had both sensitivity and specificity over 50\%. Two of these were markers for general inflammation (increases in interleukin-6 and C-reactive protein). The third was for lymphocyte count decrease. Lymphocytes are a type of white blood cell where a low count might indicate infection.

\section{How reliable are the results?}

Our confidence in the evidence from this review is low because the studies were different from each other, which made them difficult to compare. For example, some included very sick people, while some included people with hardly any COVID-19 symptoms. Also, the diagnosis of COVID-19 was confirmed in different ways: RT-PCR was sometimes used in combination with other tests.

\section{Who do the results of this review apply to?}

Routine laboratory tests can be issued by most healthcare facilities. However, our results are probably not representative of most clinical situations in which these tests are being used. Most studies included very sick people with high rates of COVID-19 virus infection of between $27 \%$ and $76 \%$. In most primary healthcare facilities, this percentage will be lower.

\section{What does this mean?}

Routine laboratory tests cannot distinguish between COVID-19 and other diseases as the cause of infection, inflammation or tissue damage. None of the tests performed well enough to be a standalone diagnostic test for COVID-19 nor to prioritize patients for treatment. They will mainly be used to provide an overall picture about the health status of the patient. The final COVID-19 diagnosis has to be made based on other tests. 


\section{How up-to-date is this review?}

We searched all COVID-19 studies up to 4 May 2020. 


\section{SUMMARY OF FINDINGS}

Summary of findings 1 . Routine laboratory tests for COVID-19: single tests

Routine laboratory tests for COVID-19: single tests

\begin{tabular}{|c|c|c|c|c|c|c|}
\hline Test & $\begin{array}{l}\text { Number of } \\
\text { studies } \\
\text { (number of } \\
\text { cases/num- } \\
\text { ber of non- } \\
\text { cases) }\end{array}$ & $\begin{array}{l}\text { Median } \\
\text { prevalence } \\
\text { (IQR) }\end{array}$ & $\begin{array}{l}\text { Specifici } \\
\text { Q1a } \\
\text { Median } a\end{array}$ & $\begin{array}{l}\text { Diagnostic } \\
\text { odds ratio } \\
(95 \% \mathrm{Cl})^{b}\end{array}$ & $\begin{array}{l}\text { Certainty } \\
\text { of the evi- } \\
\text { dencec }\end{array}$ & Interpretation of the results \\
\hline \multirow[t]{3}{*}{$\begin{array}{l}\text { White blood } \\
\text { cell count in- } \\
\text { crease }\end{array}$} & $\begin{array}{l}15 \text { studies } \\
(1262 / 5318)\end{array}$ & $\begin{array}{l}36 \% \\
\text { (25\% to } 50 \%)\end{array}$ & $78 \%$ & $\begin{array}{l}0.35(0.12 \% 60 \\
0.89) \\
(4.0 \% \\
\text { to } \\
31 \%)\end{array}$ & Very low & $\begin{array}{l}\text { WBC count increase is a general marker of inflammation, but most pa- } \\
\text { tients with COVID-19 will be missed at any cut-off value. } \\
\text { Very low-certainty evidence because of risk of bias, indirectness and in- } \\
\text { consistency }\end{array}$ \\
\hline & & & $85 \%$ & $\begin{array}{l}6.0 \% \\
(2 \% \\
\text { to } \\
17 \%)\end{array}$ & & \\
\hline & & & $92 \%$ & $\begin{array}{l}2 \% \\
(0.0 \% \\
\text { to } \\
8.0 \%)\end{array}$ & & \\
\hline
\end{tabular}




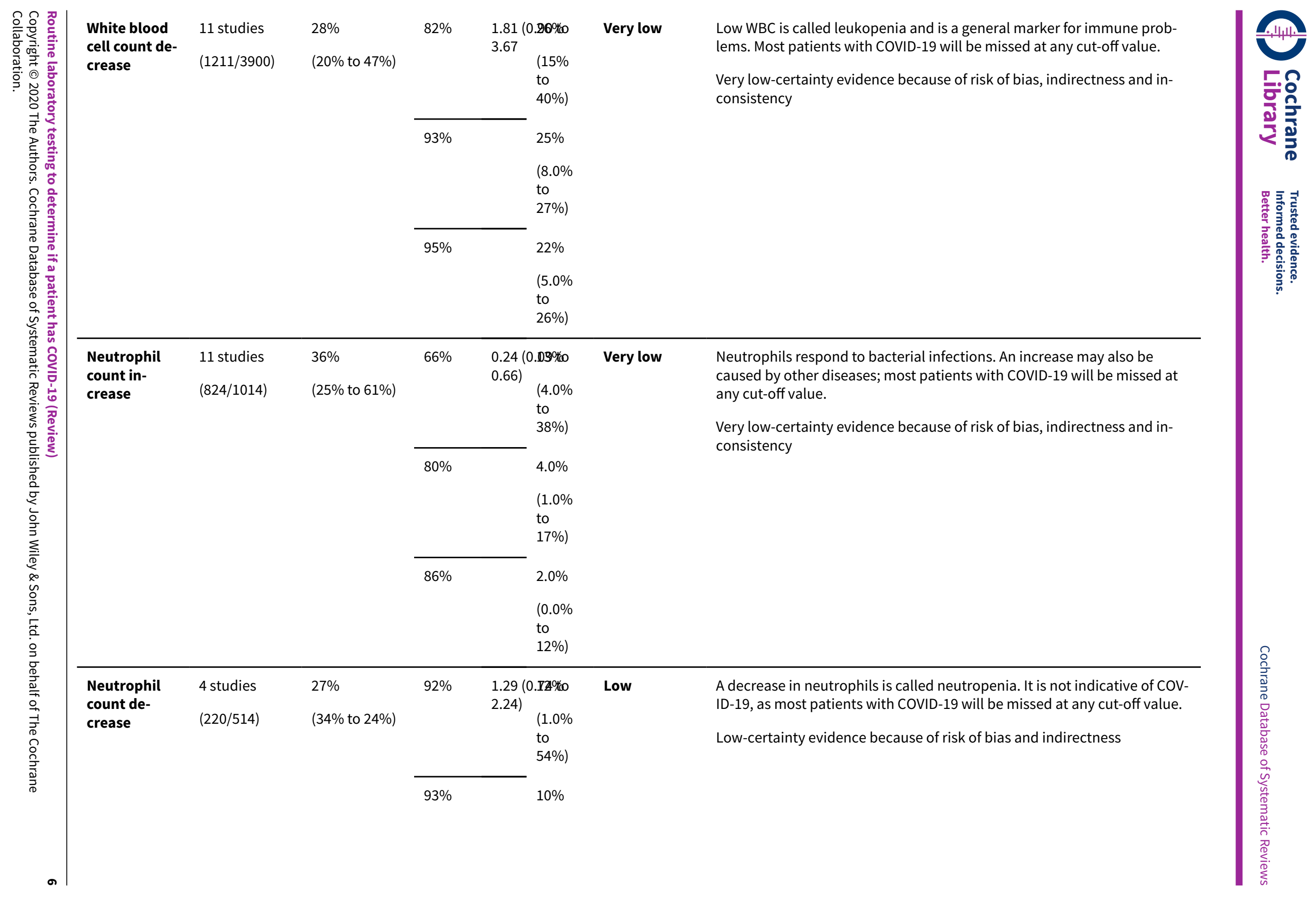




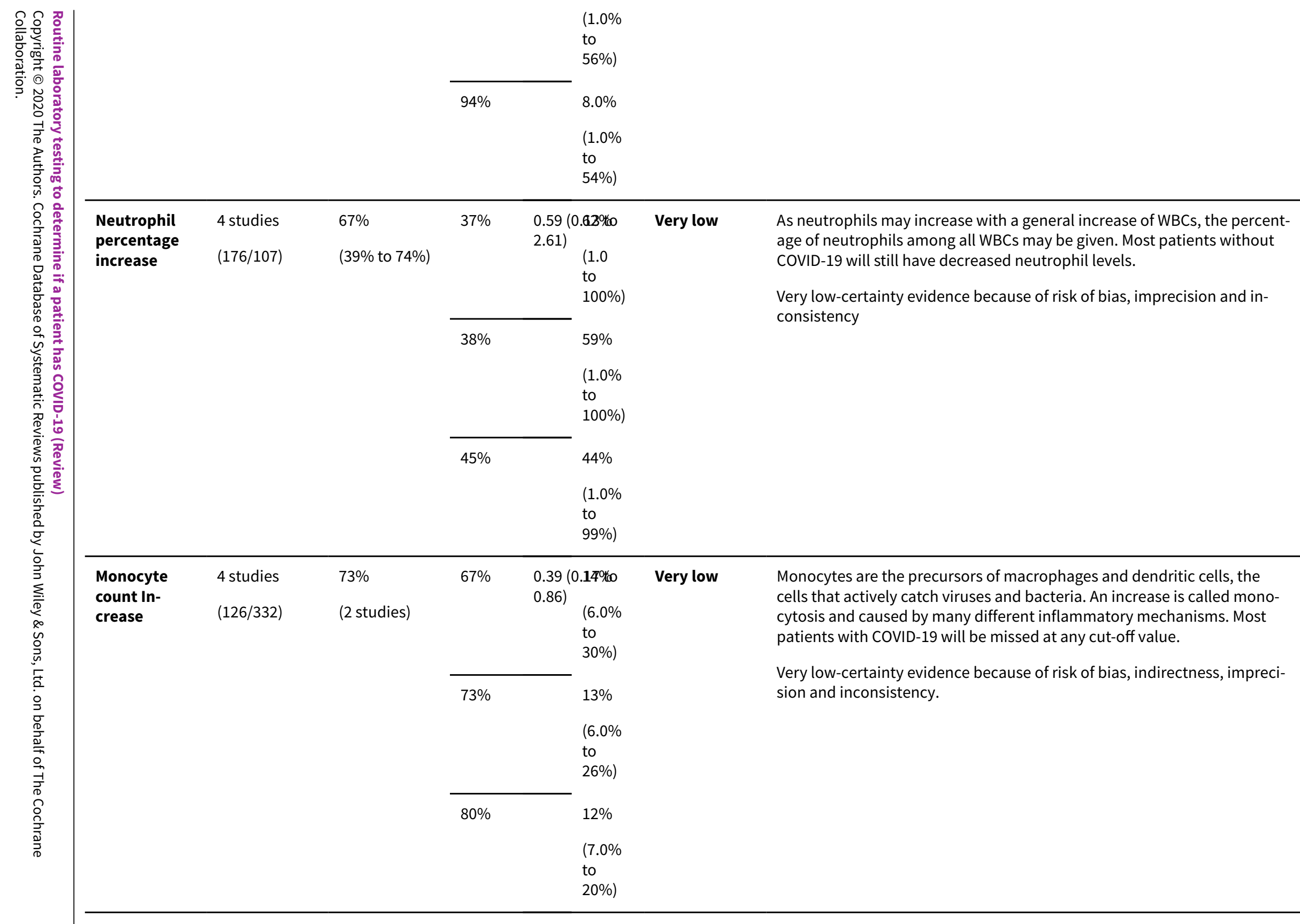




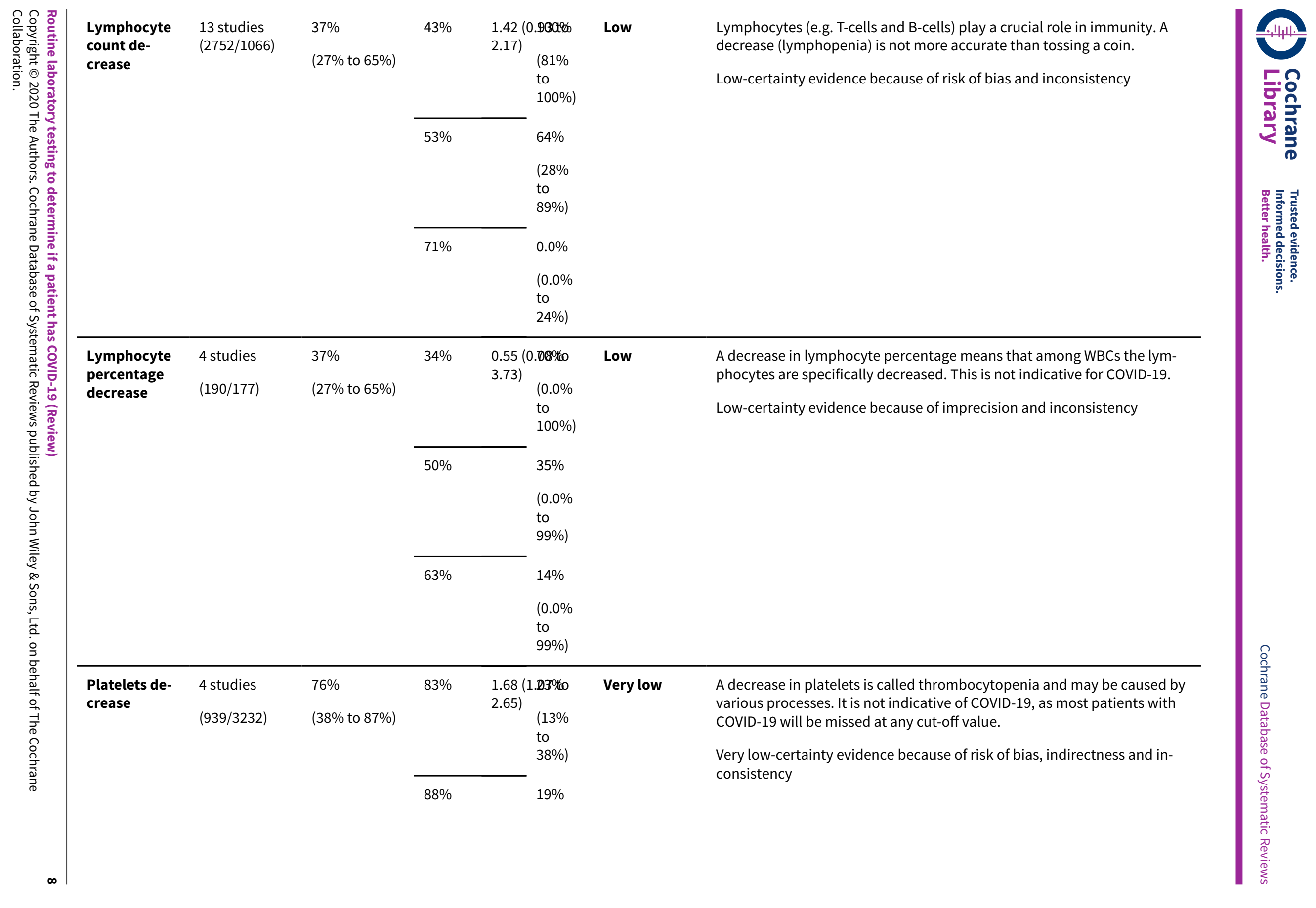




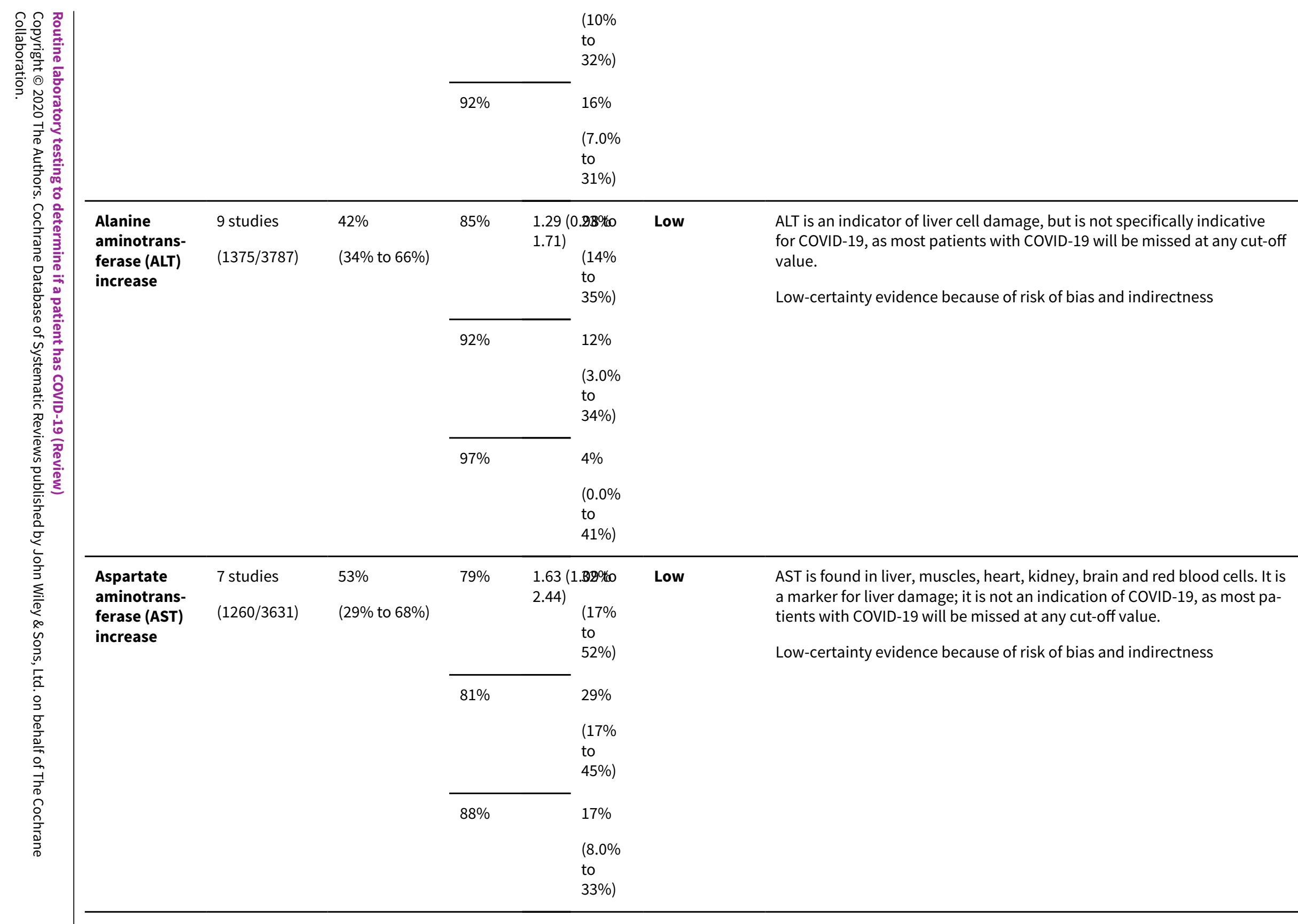




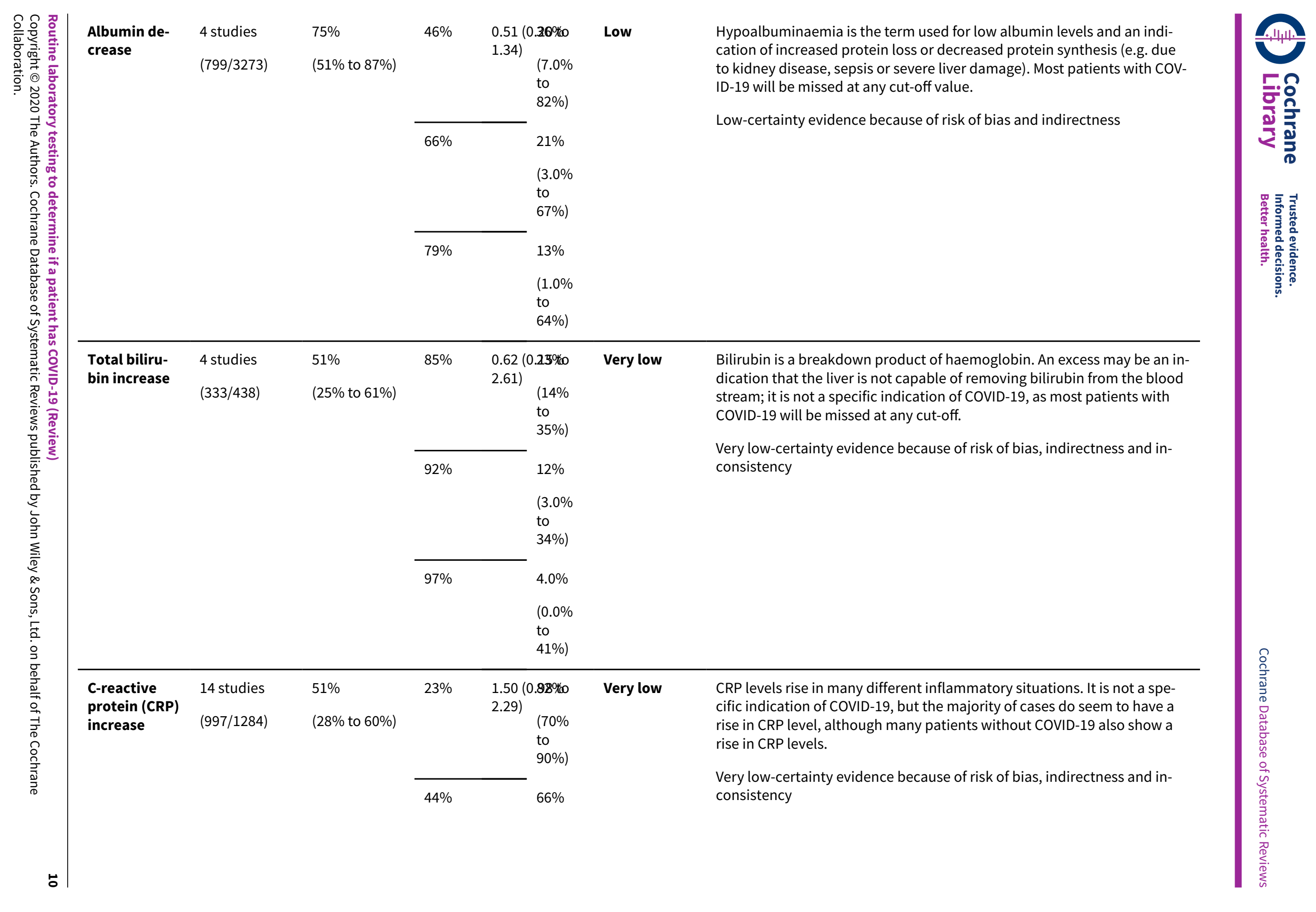




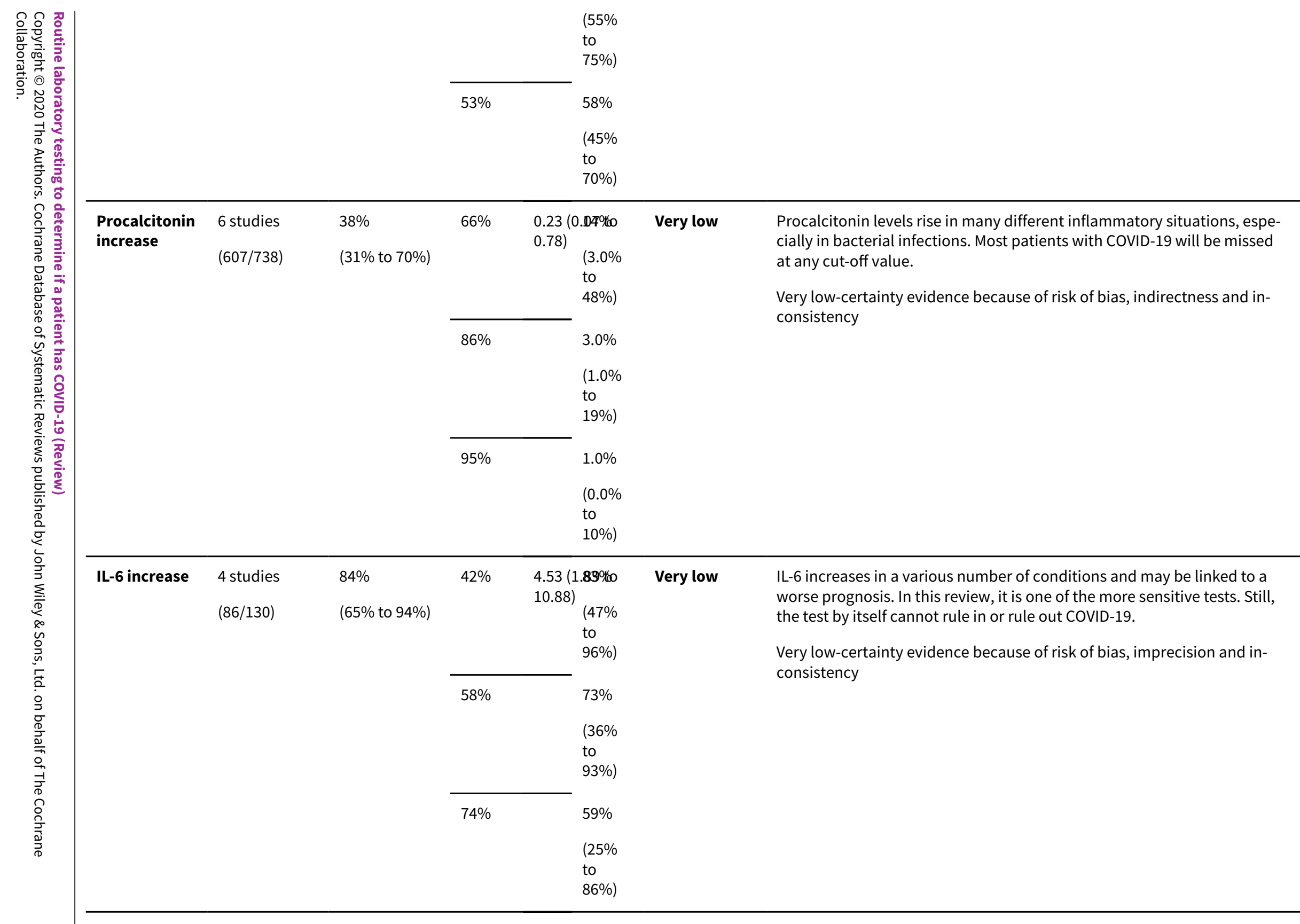




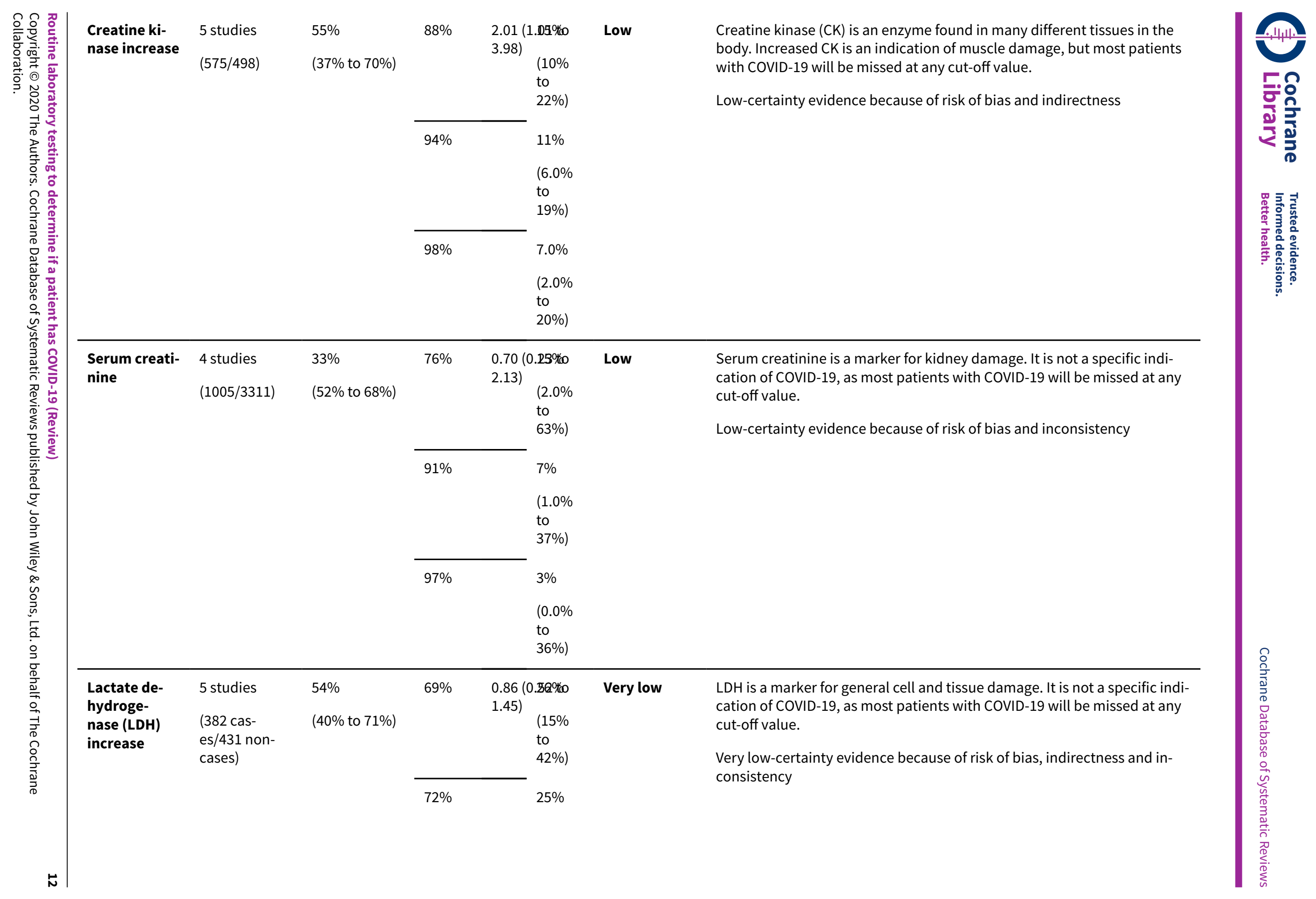




\begin{tabular}{ll} 
& \multicolumn{1}{c}{$(15 \%$} \\
& to \\
& $38 \%)$ \\
& \\
& $22 \%$ \\
$77 \%$ & $(11 \%$ \\
& to \\
& $40 \%)$
\end{tabular}

\section{GRADE Working Group grades of evidence}

High certainty: we are very confident that the true effect lies close to that of the estimate of the effect.

Moderate certainty: we are moderately confident in the effect estimate; the true effect is likely to be close to the estimate of the effect, but there is a possibility that it is substantially different.

Low certainty: our confidence in the effect estimate is limited; the true effect may be substantially different from the estimate of the effect.

Very low certainty: we have very little confidence in the effect estimate; the true effect is likely to be substantially different from the estimate of effect.

ALT: alanine aminotransferase; AST: aspartate aminotransferase; CI: confidence interval; CRP: C-reactive protein; IL-6: interleukin-6; IQR: interquartile range; LDH: lactate dehydrogenase; WBC: white blood cell. Included studies defined a positive test result as an increase or a decrease compared to normal range values, or both.

aThe specificity marking the first quartile (Q1) of all specificities of the studies included, the median specificity, and the third quartile (Q3) specificity were used to estimate the corresponding sensitivity estimates from the HSROC model.

bA sensitivity and specificity both of $70 \%$ would lead to a diagnostic odds ratio of 5.0

'Starting at high certainty of the evidence, the evidence was downgraded by one level when at least half of the studies had high risk of bias on one or more domains; downgraded for indirectness when at least half of the studies in the meta-analyses had high concerns regarding applicability on at least one domain; downgraded for imprecision when fewer people with the target condition were included then would have been needed to achieve the sensitivity-estimates listed with a width of the confidence interval of at most $10 \%$ points; and downgraded for inconsistency when study estimates differed more than $20 \%$ points from each other. Publication bias was not considered to be a problem.

Summary of findings 2. Comparisons of routine laboratory tests for COVID-19 with sensitivity and specificity higher than $\mathbf{5 0} \%$

Comparisons of routine laboratory tests for COVID-19 with sensitivity and specificity higher than $50 \%$

\begin{tabular}{|c|c|c|c|c|c|c|c|c|}
\hline & $\begin{array}{l}\text { Number of studies } \\
\text { (number of cas- } \\
\text { es/number of non- } \\
\text { cases) }\end{array}$ & $\begin{array}{l}\text { Fixed speci- } \\
\text { ficity }\end{array}$ & $\begin{array}{l}\text { Summary sensitivity } \\
\text { corresponding with } \\
\text { fixed specificity } \\
(95 \% \mathrm{Cl})\end{array}$ & \multicolumn{5}{|c|}{$\begin{array}{l}\text { Interpretation of the results: tests used in a hypothetical cohort of } \\
1000 \text { people tested for COVID-19, at a pre-test probability of } 5 \% \text { and } \\
36 \% a\end{array}$} \\
\hline $\begin{array}{l}\text { Lymphocyte Count De- } \\
\text { creaseb }^{\text {b }}\end{array}$ & 13 studies & $53 \%$ & $64 \%$ & 0.05 & 32 & 447 & 18 & 504 \\
\hline
\end{tabular}




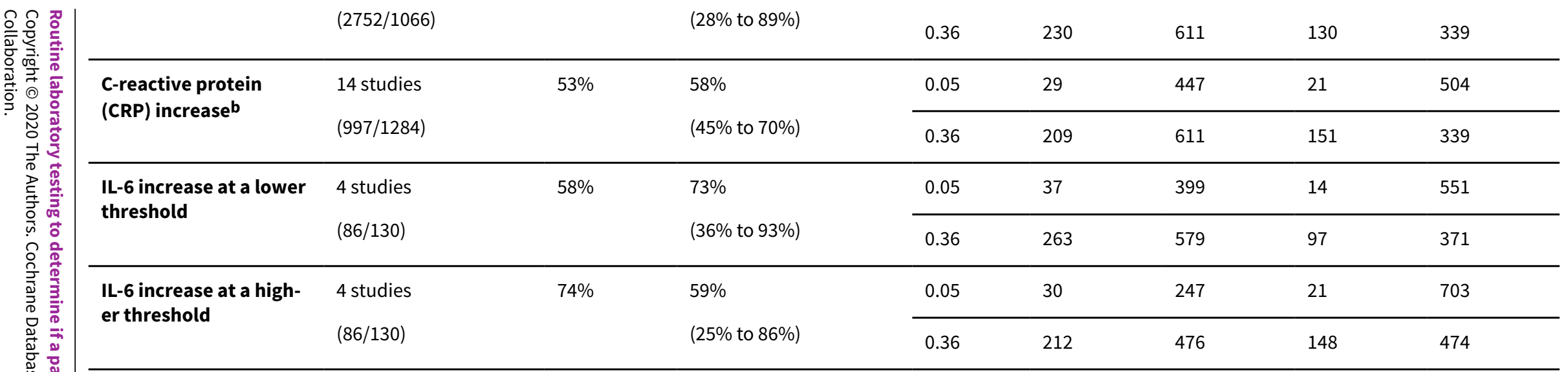

Cl: confidence interval; FN: false negative; FP: false positive; TN: true negative; TP: true positive. Included studies defined a positive test result as an increase or a decrease compared to normal range values, or both.

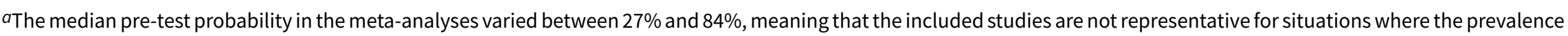
is $5 \%$ or lower. The median prevalence over all the single-gate studies was $36 \%$.

bThe direct comparison between lymphocyte count increase and C-reactive protein (CRP) increase ( 9 studies) showed that CRP was considerably more accurate than lymphocyte count increase: relative diagnostic odds ratio (DOR) was 2.02 ( $95 \%$ confidence interval 1.47 to 2.78 ). As the confidence intervals of all the DORs in the indirect comparisons included a non-informative value (i.e. DOR $=1$ ), a relative DOR of 2 does not mean the alternative is much more informative. 


\section{B A C K G R O U N D}

On 30 December 2019, a cluster of patients with pneumonia of unknown origin in Wuhan, China, was publicly reported via ProMED (promedmail.org/promed-posts). In January 2020, it became clear that this was caused by a new coronavirus and that it was spreading to other countries as well. In March 2020, the World Health Organization (WHO) declared the severe acute respiratory syndrome coronavirus 2 (SARS-CoV-2) and resulting COVID-19 a worldwide pandemic. This pandemic, in combination with the novelty of the virus, presents important diagnostic challenges.

These challenges range from understanding the value of signs and symptoms in predicting possible infection, assessing whether existing biochemical and imaging tests can identify infection and patients who need critical care, and evaluating whether new diagnostic tests can provide accurate rapid and point-of care testing, either to identify current infection, rule out infection, identify people in need of care escalation, or to test for past infection and immunity.

This review follows a generic protocol that covers the full series of Cochrane diagnostic test accuracy (DTA) reviews for the diagnosis of COVID-19 (Deeks 2020b). The Background and Methods sections of this review therefore use some text that was originally published in the protocol, and text that overlaps some of our other reviews (Deeks 2020a; Dinnes 2020; Struyf 2020).

The present review concentrates on the diagnostic accuracy of routine laboratory testing as a triage test to determine if a person has COVID-19 pneumonia or SARS-CoV-2 infection, and to facilitate further testing. In clinical care, routine laboratory markers such as white blood cell count, measures of anticoagulation, C-reactive protein (CRP) and procalcitonin, are used to assess the health status of a patient. These laboratory markers are also used in patients with COVID-19 infection and may be useful for triage of people with potential COVID-19 infection for treatment or more intensive treatment, especially in situations where time and resources are limited.

\section{Target condition being diagnosed}

COVID-19 is the disease caused by infection with SARS-CoV-2. The key target condition for this review was current COVID-19. SARS-CoV-2 infection can be asymptomatic (no symptoms); mild or moderate (symptoms such as fever, cough, aches, lethargy but without difficulty breathing at rest); severe (symptoms include breathlessness and increased respiratory rate indicative of pneumonia); or critical (requiring respiratory support due to severe acute respiratory syndrome (SARS) or acute respiratory distress syndrome (ARDS)). People with COVID-19 pneumonia (severe or critical disease) require distinctive patient management, and it is important to be able to identify these patients.

In this review, we focus on COVID-19, without making the distinction between mild to moderate and severe disease.

\section{Index test(s)}

We collated evidence on all routine biomarker tests reported in the identified studies. These can be classified into:

- full blood count, haemoglobin and red blood cells;

- coagulation markers;
- liver markers, cardiac markers and kidney function markers;

- general inflammatory markers; and

- metabolic markers.

\section{Clinical pathway}

Decisions about patient and isolation pathways for COVID-19 vary according to health services and settings, available resources, and stages of the epidemic. They will change over time if and when effective treatments and vaccines are identified. The decision points between these pathways vary, but all include points at which knowledge of the accuracy of diagnostic information is needed to be able to inform rational decisions.

Standard workup for individuals suspected of COVID-19 infection consists of assessing signs and symptoms and a polymerase chain reaction (PCR) test. It is common practice that, when patients enter (either outpatient or admission) the hospital, they will generally have routine laboratory tests done.

Routinely available tests for infection and inflammation may be considered in the investigation of people with possible COVID-19 infection. For example, many healthcare facilities have access to standard laboratory tests for infection, such as CRP, procalcitonin, measures of anticoagulation, and white blood cell count with leukocyte differentiation. Routine laboratory markers may be used as a triage test, either on their own, or in combination with signs and symptoms. In low-resource settings, they may sometimes even be the only tests available. In order to function as a triage test or stand-alone test, a high sensitivity is needed, to prevent infected patients from being sent home or into a general ward with uninfected patients. For a triage test, specificity may be less important, as positive tests will be further investigated. Also, routine laboratory tests may be used to tip the decision to treat the patient as having COVID-19 or not in case of mixed results from other tests or where a definite diagnosis cannot be made. In that case, knowledge of the sensitivity and specificity in a particular (pre-tested) patient population may be useful. Routine laboratory tests may also be used in the further diagnostic workup, to predict mild versus severe outcomes, or to monitor treatment response. These aims of testing will not be the focus of this systematic review.

\section{Alternative test(s)}

The test that is believed to be most accurate in detecting SARS$\mathrm{CoV}-2$ is reverse transcriptase polymerase chain reaction (RT-PCR). In many settings, this test will be available, but the results take time before they become available. Although rapid antigen and molecular-based tests are also available, the value of these rapid tests is still not clear. Antibody tests provide insights into the antibody response, but may also take a few days before the response is detectable and therefore the results are available.

Alternatives to routine laboratory tests may depend on the setting and situation where the tests are done. For example, in primary care, alternatives may consist of signs and symptoms and rapid and point-of-care tests. Similarly, point-of-care ultrasound may be used, if resources allow. The benefit of routine laboratory tests (and of signs and symptoms) may be as an indication of the severity of a disease: a value further from the reference values may indicate more severe infections. 
In emergency departments, chest X-ray, ultrasound, and computed tomography (CT) are widely used diagnostic imaging tests to identify COVID-19 pneumonia. Which imaging test is available may depend on the type of hospital and available resources: a tertiary care hospital in a high-income country may have a mobile CT scan available, while in smaller hospitals only X-ray and ultrasound are accessible. These imaging tests have the advantage that the condition of the lungs can be assessed visually.

These other tests are all addressed in the other Cochrane DTA reviews in this suite of reviews (Deeks 2020a; Dinnes 2020; McInnes 2020; Struyf 2020).

\section{Rationale}

It is essential to understand the accuracy of tests and diagnostic features to identify how they can be used optimally in different settings to develop effective diagnostic and management pathways. New evidence about routine laboratory testing is becoming available quickly. Therefore, we have produced a Cochrane 'living systematic review' (a systematic review that is continually updated, incorporating relevant new evidence as it becomes available) that will summarize new and existing evidence on the clinical accuracy of routine laboratory markers. Estimates of accuracy from this review will help inform diagnostic, screening, and patient management decisions.

\section{OB JECTIVES}

To assess the diagnostic accuracy of routine laboratory testing as a triage test to determine if a person has COVID-19.

\section{Secondary objectives}

Where data are available, we investigated the accuracy (either by stratified analysis or meta-regression) according to a specific measurement or test, days of symptoms, severity of symptoms, reference standard, sample type, study design, and setting.

\section{METHODS}

\section{Criteria for considering studies for this review Types of studies}

We kept the eligibility criteria broad to include all patient groups and all variations of a test (that is, if patient population was unclear, we included the study).

We included studies of all designs that produce estimates of test accuracy or provide data from which estimates can be computed: cross-sectional studies, case-control designs and consecutive series of patients assessing the diagnostic accuracy of routine laboratory testing as a triage test to determine if a person has COVID-19.

We intended to include studies recruiting only COVID-19 cases, to estimate sensitivity, or those restricted to people without COVID-19, to estimate specificity (Deeks 2020a). We decided to deviate from this rule as the added value of such studies for our review is questionable. We included both single-gate designs, where a single group of participants, often suspected of having the target condition, is recruited, and multi-gate designs, where people with and without the target condition are recruited separately. We Intended to include studies that based their results on individual patients as well as studies that based their results on samples. We carefully considered the limitations of different study designs, using quality assessment and analysis.

\section{Participants}

We included studies recruiting people presenting with suspected SARS-CoV-2 infection, studies that recruited people to screen for disease, and studies based on serum banks created from known cases of COVID-19 and controls.

Studies had to include a minimum of 10 samples or 10 participants.

\section{Index tests}

We collected evidence on all routine biomarker tests reported in the identified studies. We interpreted the term 'routine' broadly, considering that some markers will be more routine in some settings or countries than in others. Test positivity could have been defined as an increase in values compared to the normal ranges, or as a decrease compared to normal values.

\section{Target conditions}

To be eligible, studies needed to identify at least one of:

- current SARS-CoV-2 infection;

- COVID-19 pneumonia.

\section{Reference standards}

Reverse transcriptase polymerase chain reaction (RT-PCR) is considered the best available test, although due to rapidly evolving knowledge about the target conditions, multiple reference standards on their own as well as in combination have emerged.

Therefore, we included the following reference standards:

- RT-PCR alone;

- RT-PCR, clinical expertise, and imaging (for example, CT thorax);

- repeated RT-PCR several days apart or from different samples;

- plaque reduction neutralization test (PRNT) or enzyme-linked immunosorbent assay (ELISA);

- information available at a subsequent time point;

- WHO (Appendix 1), and other case definitions;

- any other reference standard used by study authors.

\section{Search methods for identification of studies}

\section{Electronic searches}

We conducted a single literature search to cover our suite of Cochrane COVID-19 diagnostic test accuracy (DTA) reviews (Deeks 2020b; McInnes 2020).

We conducted electronic searches using two primary sources. Both of these searches aimed to identify all published articles and preprints related to COVID-19, and were not restricted to those evaluating tests. Thus, there are no test terms, diagnosis terms, or methodological terms in the searches. Searches were limited to 2019 and 2020, and for this version of the review have been conducted to 4 May 2020. 


\section{Cochrane COVID-19 Study Register searches}

We used the Cochrane COVID-19 Study Register (covid-19.cochrane.org), for searches conducted to 28 March 2020. At that time, the register was populated by searches of PubMed, as well as trials registers at ClinicalTrials.gov and the WHO International Clinical Trials Registry Platform (ICTRP).

Search strategies were designed for maximum sensitivity, to retrieve all human studies on COVID-19 and with no language limits (Appendix 2).

\section{COVID-19 Living Evidence Database from the University of Bern}

From 28 March 2020, we used the COVID-19 Living Evidence database from the Institute of Social and Preventive Medicine (ISPM) at the University of Bern (www.ispm.unibe.ch), as the primary source of records for the Cochrane COVID-19 DTA reviews. This search includes PubMed, Embase, and preprints indexed in bioRxiv and medRxiv databases. The strategies as described on the ISPM website are described here (ispmbern.github.io/covid-19/; Appendix 3).

The decision to focus primarily on the 'Bern' feed was due to the exceptionally large numbers of COVID-19 studies available only as preprints. The Cochrane COVID-19 Study Register has undergone a number of iterations since the end of March and we anticipate moving back to the Register as the primary source of records for subsequent review updates.

\section{Searching other resources}

We identified Embase records obtained through Martha Knuth for the Centers for Disease Control and Prevention (CDC), Stephen B Thacker CDC Library, COVID-19 Research Articles Downloadable Database (cdc.gov/library/researchguides/2019novelcoronavirus/ researcharticles.html), and de-duplicated them against the Cochrane COVID-19 Study Register up to 1 April 2020.

We also checked our search results against two additional repositories of COVID-19 publications including:

- the Evidence for Policy and Practice Information and Coordinating Centre (EPPI-Centre) 'COVID-19: Living map of the evidence' (eppi.ioe.ac.uk/COVID19_MAP/covid_map_v4.html);

- the Norwegian Institute of Public Health 'NIPH systematic and living map on COVID-19 evidence' (www.nornesk.no/ forskningskart/NIPH_diagnosisMap.html).

Both of these repositories allow their contents to be filtered according to studies potentially relating to diagnosis, and both have agreed to provide us with updates of new diagnosis studies added. For this iteration of the review, we examined all diagnosis studies from either source up to 4 May 2020.

We did not apply any language restrictions.

\section{Data collection and analysis}

\section{Selection of studies}

First, all retrieved articles were screened by an overall team of screeners who divided the articles over the different rapid DTA reviews. Then, the set of studies possibly involving routine laboratory markers was imported into Covidence. Two review authors screened each title and abstract independent of each other for possible inclusion. In the next step, two review authors independently screened the full text of each possibly relevant article. For articles only available in languages other than English, we used Google Translate and review authors who could read and understand that language. We solved disagreements by discussion. If discussion could not solve the dispute, we consulted a third review author.

\section{Data extraction and management}

Two review authors carried out data extraction for each study. We assigned multiple studies with first authors with the same last name to one extractor, so that they could detect preprints from already peer-reviewed, published articles. We contacted study authors when we needed to check details and obtain missing information. Data were extracted on the country and region, the setting, the time period of the study, funding, and information needed for the Characteristics of included studies tables. Studies may have defined a positive test result as a decrease compared to normal vaues, as an increase compared to normal values, and as both increase and decrease. Where possible, we adapted the twoby-two tables in such a way that all studies included in the analyses reported on the same test positivity definition. However, if studies reported both in- and decrease as a positive test result, we included both. We resolved disagreements by discussion between the two review authors, and two other review authors checked the results when these were entered into Review Manager 5.4 (Review Manager 2020).

\section{Assessment of methodological quality}

\section{QUADAS-2 assessment}

Two review authors independently assessed risk of bias and applicability concerns using the Quality Assessment of Diagnostic Accuracy Studies (QUADAS-2) tool (Table 1). We resolved disagreements by discussion between three review authors.

QUADAS-2 facilitates assessment across four domains: patient selection, index test, reference standard and flow and timing (Whiting 2011). Each domain is assessed in terms of risk of bias and the first three domains are also assessed in terms of concerns regarding applicability. Signalling questions are included to help judge bias. Table 1 shows the definitions used for assessing the methodological quality.

\section{Statistical analysis and data synthesis}

Most routine laboratory tests provide test results as continuous measurements. That means that an explicit threshold is needed to provide positive and negative results for estimation of sensitivity and specificity. Some tests indicate disease if the value is decreased relative to the normal ranges, for other tests disease is indicated when the value is increased, and for some tests, both increase and decrease may indicate the presence of disease. For each test in each study, we reported the threshold used in our analyses, and whether an increase or a decrease in value was regarded as a positive test result.

From each study, we included one threshold for each test. If multiple thresholds were reported, we chose the threshold that was most often used in the other studies. We presented the resulting sensitivity and specificity in forest plots. We reported median and interquartile range (IQR) of pre-test probability of the target condition in $2 \times 2$ tables from single-gate studies. 
We considered a meta-analysis appropriate when four or more studies reported on a particular test. As studies reported mostly different thresholds for the same test, we used the Hierarchical Summary Receiver Operator Curve (HSROC) model for metaanalyses to estimate summary curves, as recommended by the Cochrane Handbook for Systematic Reviews of Diagnostic Test Accuracy (Macaskill 2010). Since summary sensitivities and specificities are only clinically interpretable when the studies included in a meta-analysis use a common cut-off, we estimated sensitivity at points on the SROC curves corresponding to the median specificity observed in the studies included in the metaanalysis. The 'Summary of findings' table also reported the estimates for the first and third quartile specificity. Meta-analyses were undertaken in SAS 9.4, using PROC NLMIXED (SAS 2015).

In resource-limited situations, or in case SARS-CoV-2-specific tests are not available, routine laboratory tests may be the only tests available. In order to identify the most discriminative test in such a situation, we compared the diagnostic accuracy of biomarkers that had at least a sensitivity of $50 \%$ at a minimum specificity of $50 \%$ (either median or IQR). We performed these analyses on all studies that evaluated one of these tests (indirect comparison). We performed additional analyses restricted to studies that made head-to-head comparisons (i.e. assessed two of the biomarkers in the same participants) when at least four studies were included that enabled these direct comparisons. We made test comparisons by adding a covariate for test type to the HSROC model to assess the effect of test type on the accuracy, cut-off or shape parameters of the model. In addition, whenever the estimated SROC curves had the same shape, we calculated the relative diagnostic odds ratio (RDOR) as a summary of the relative accuracy of two biomarkers at hand. To assess the statistical significance of differences in test accuracy, we used likelihood ratio tests for comparisons of models with and without covariate terms. If too few primary studies $(n<10)$ were available for the head-to-head comparison, we assumed the shape parameter of the model to be equal for the biomarkers under evaluation.

\section{Investigations of heterogeneity}

We investigated sources of heterogeneity if adequate data were available, as listed in the Secondary objectives, either using stratification (where we believed it was inappropriate to combine studies) or through meta-regression models.

\section{Summary of findings and assessment of the certainty of the evidence}

We developed a list of key findings in 'Summary of findings' tables and determined the certainty in the summary estimates for each test and findings, using the GRADE approach (Schünemann 2020a; Schünemann 2020b. Starting at high certainty, we downgraded meta-analyses by one level when at least half of the studies had high risk of bias on one or more domains; we downgraded for indirectness when at least half of the studies in the meta-analyses had high concerns regarding applicability on at least one domain; we downgraded for imprecision when fewer people with the target condition were included than would have been needed to achieve the sensitivity estimates listed, with a width of the confidence interval of at most 10 percentage points; and we downgraded for inconsistency when study estimates differed more than 20 percentage points from each other. We did not consider publication bias to be a problem.

\section{Updating}

We will undertake the searches of published literature, preprints, and new test approvals weekly, and, dependent on the number of new and important studies found, we will consider updating each review with each search if resources allow.

\section{RE S U L T S \\ Results of the search}

The overall search for all reviews in this suite was done on 4 May 2020 and resulted in 10,965 records. The first selection resulted in 651 records that were potentially eligible for this review of routine laboratory tests. After title and abstract screening, we excluded 239 records leaving 412 to be assessed on full text (Figure 1). Of these, we removed 17 duplicates and preprints, 31 studies that were not in the scope of the review, 66 studies that did not contain original data and 7 studies that were retracted or otherwise no longer available. Of the remaining 291 studies, 246 studies only considered proven cases of COVID-19. These reported percentages of proven patients that had an increased or decreased biomarker level. We decided not to extract these data, as only the sensitivity of these markers would be estimable. Furthermore, the aim of these excluded studies was not to assess the accuracy of routine markers for COVID-19, but just to describe the findings or to assess the accuracy of markers to distinguish between mild and severe disease. 
Figure 1. Study flow diagram. Studies were retrieved in a combined search process for all DTA reviews about tests for COVID-19 and then divided over the different review teams. Due to this process, some preprints only came to light after the data-extraction phase

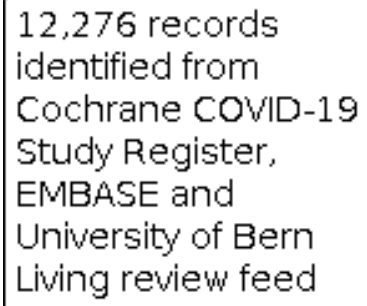

62 additional records identified through EPPI-centre and Norway living review feeds

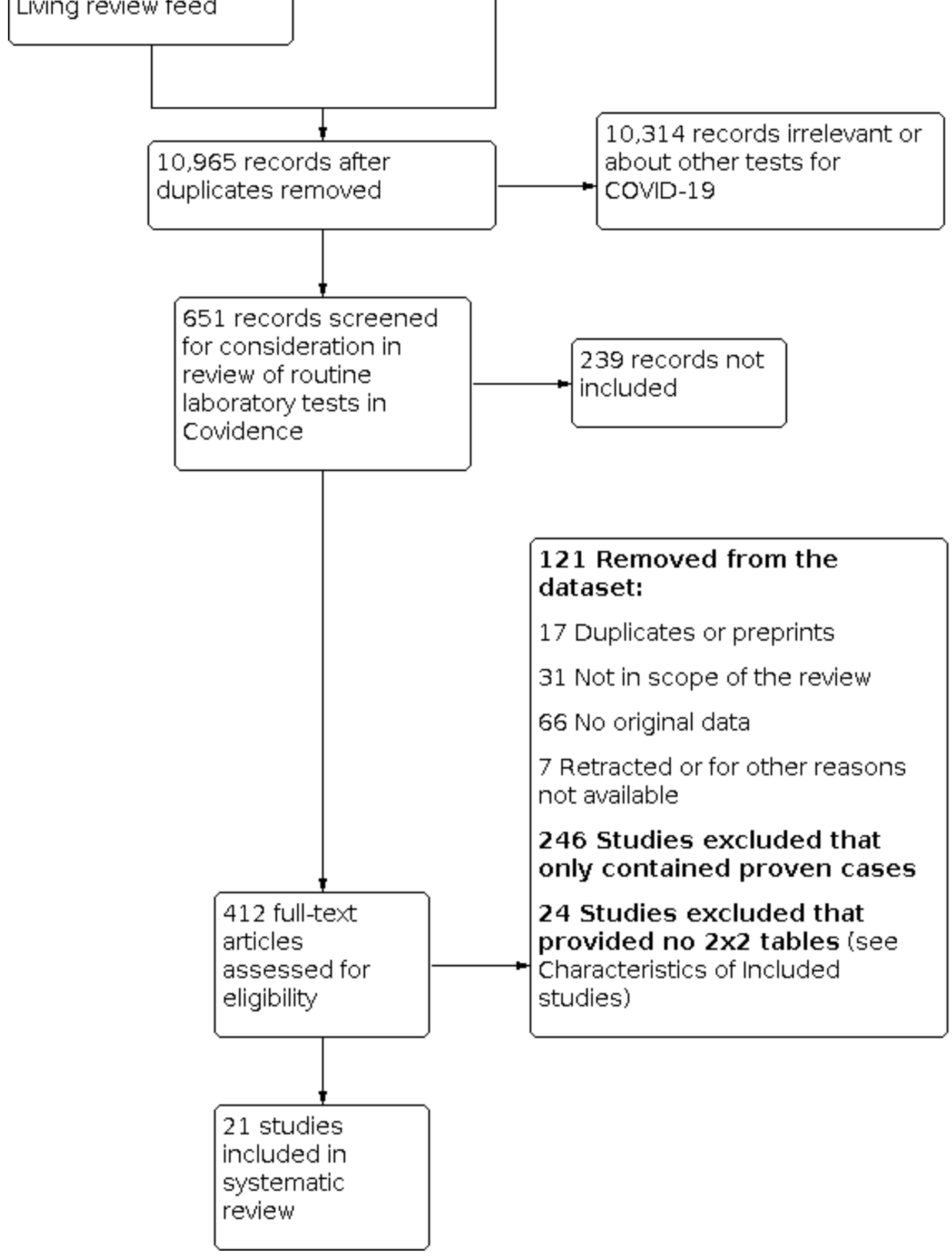


The Characteristics of excluded studies table lists the 24 studies that included both patients with and without the target condition, but provided insufficient data to construct $2 \times 2$ tables to estimate sensitivity and specificity.

The remaining 21 studies are included in this review.

\section{Included studies}

Of the 21 included studies, 14 were single-gate studies (a study including patients with suspected COVID-19), six were multiplegate studies (including proven COVID-19 patients and separately one or more groups of non-COVID-19 patients). In the remaining study the design was unclear (Characteristics of included studies).

The included studies comprised in total 14,126 COVID-19 patients and 56,585 people without COVID-19. They included a total of 67 laboratory tests (Table 2). Four studies included a mix of children and adults, 16 included only adults and one study was only in children. Seventeen studies were done in China, and one each in Iran, Italy, Taiwan and the USA. Nine studies included patients in general hospitals, six studies included patients in emergency departments, three studies included patients in fever clinics, and the remaining three studies included patients in a paediatric hospital, tertiary hospitals, and in veterans affairs databases.

Thirteen studies used RT-PCR as reference standard, three studies used other nucleic acid tests, one combined RT-PCT and chest CT, one used a 'pharyngeal swab' (unclear for which test), one combined RT-PCR, signs and symptoms and chest CT, one used a non-specific SARS-CoV-2 assay, and one based diagnosis on the Diagnosis and Treatment Program of New Coronavirus Pneumonia,
China National Health Commission of the People's Republic of China (CDC) case definition (sixth trial version). The target condition was SARS-CoV-2 infection in 17 studies, and SARS-Cov-2 pneumonia in two studies and COVID-19 in two other studies.

Eight studies were prepublications and 13 were published in peer reviewed journals.

\section{Methodological quality of included studies}

Of the 21 studies, four studies had low or unclear risk of bias on all domains; all other studies had high risk of bias for at least one domain (Figure 2). Six studies had low concerns regarding applicability for all domains. Eleven studies were judged to have a high risk of bias with respect to the patient selection domain, mainly because of including separate groups of cases and noncases. Six studies did not describe the order of inclusion of their participants and two did not include a random or consecutive sample. Five studies were case-control designs and in two studies the design was unclear. We judged risk of bias for patient selection unclear in four studies. We judged three studies as having a high risk of bias regarding the index test. In these studies the index test was either interpreted with knowledge of the reference standard or there was no predefined cut-off value. Fourteen studies used RTPCR as a reference standard for SARS-CoV-2 as a target condition, and three used RT-PCR as a reference standard with COVID-19 as a target condition. Only four studies reported multiple tests (e.g. RTPCR and CT scans) or criteria (e.g. the criteria of the National Health Commission China) as a reference standard for COVID-19 as a target condition. Flow and timing was unclear in the majority of studies ( $\mathrm{n}$ $=12$ ), because the time between the reference standard and index test was unclear. 
Figure 2. Risk of bias and applicability concerns summary: review authors' judgements about each domain for each included study

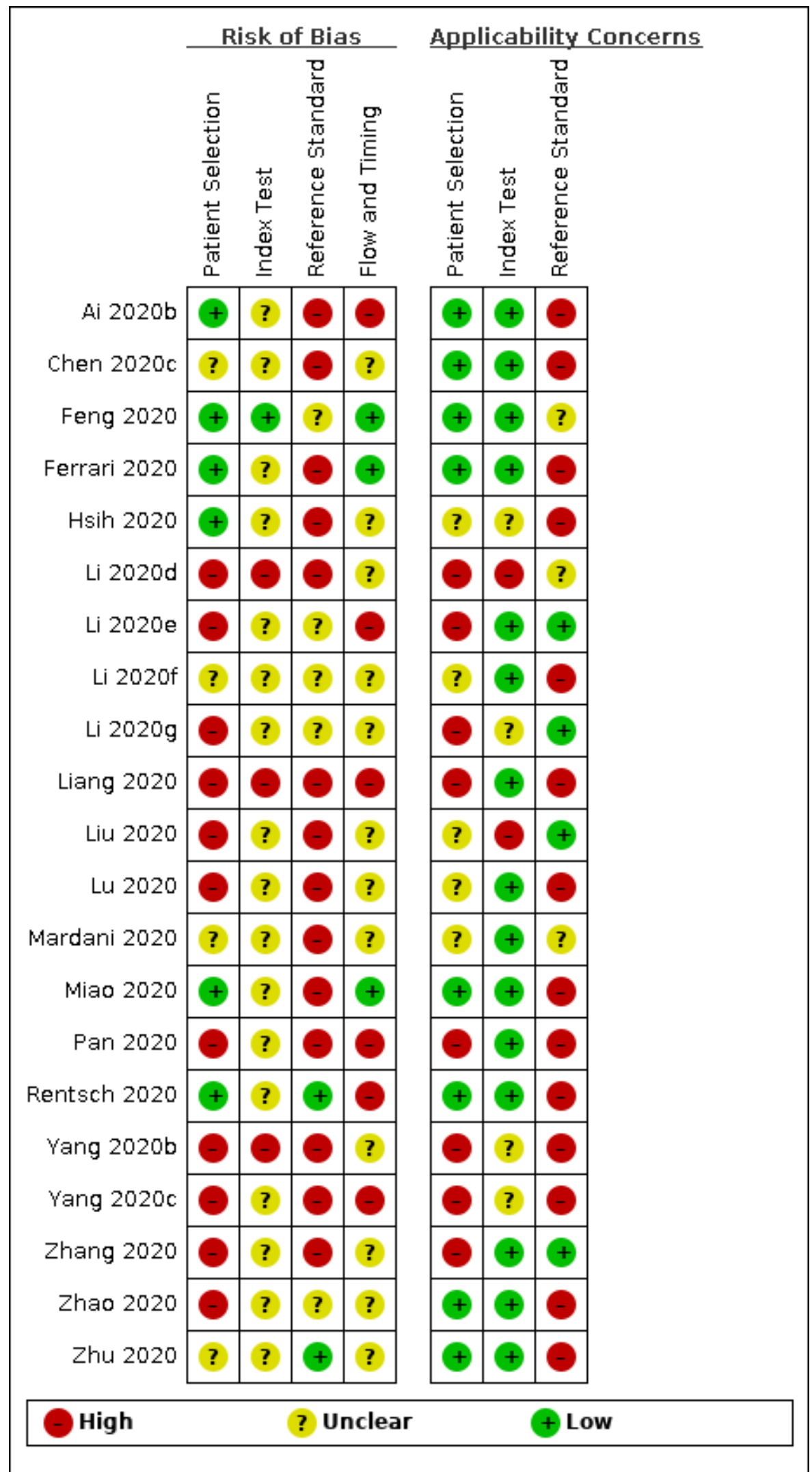

Routine laboratory testing to determine if a patient has COVID-19 (Review) 
None of the studies had low concerns regarding applicability for all domains. As the index test consisted of routine laboratory measurements, these were considered to be low concerns regarding applicability for most studies. In some cases, studies used different cut-off values, leading to high concerns regarding applicability. As the focus of our review was COVID-19, we assessed the 14 studies that only used RT-PCR as a reference standard as high concerns regarding applicability of the reference standard.

\section{Findings}

Below we describe the findings for tests assessed in four or more studies: white blood cell count increase and decrease, neutrophil count increase and decrease, monocyte count increase, lymphocyte count decrease, platelets decrease, alanine aminotransferase increase, aspartate aminotransferase increase, albumin decrease, total bilirubin, CRP increase, procalcitonin increase, IL- 6 increase, creatine kinase increase, serum creatinine and lactate dehydrogenase increase. See Table 2 for an overview of tests and cut-off values per study. Summary of findings 1 shows the summary of findings for the individual tests, including sensitivity, specificity and diagnostic odds ratios (DORs). All HSROC curves were close to the non-informative diagonal, with DORs varying between 0.23 (95\% confidence interval $(\mathrm{Cl}) 0.07$ to 0.78 ) and 4.53 ( $95 \% \mathrm{Cl} 1.89$ to 10.88). As an indication, a test with a sensitivity of $70 \%$ and a specificity of $70 \%$ has a DOR of 5.0 .

\section{Complete blood count \\ White blood cell count increase}

Fifteen studies (1262 cases/5318 non-cases) reported on white blood cell count increase (Figure 3 ). The cut-off values for an increase in white blood cell count varied from $9.5 \times 10^{9}$ cells/L to $11.2 \times 10^{9}$ cells/L, with the exception of one study that used a cutoff value of $6.4 \times 10^{9}$ cells/L. The median prevalence of COVID-19 in the 12 single-gate studies that reported on white blood cell count increase was 36\% (IQR 25\% to 50\%). 
Figure 3. Summary ROC plot of tests. 1: white blood cell count (WBC) increase; 2: WBC decrease

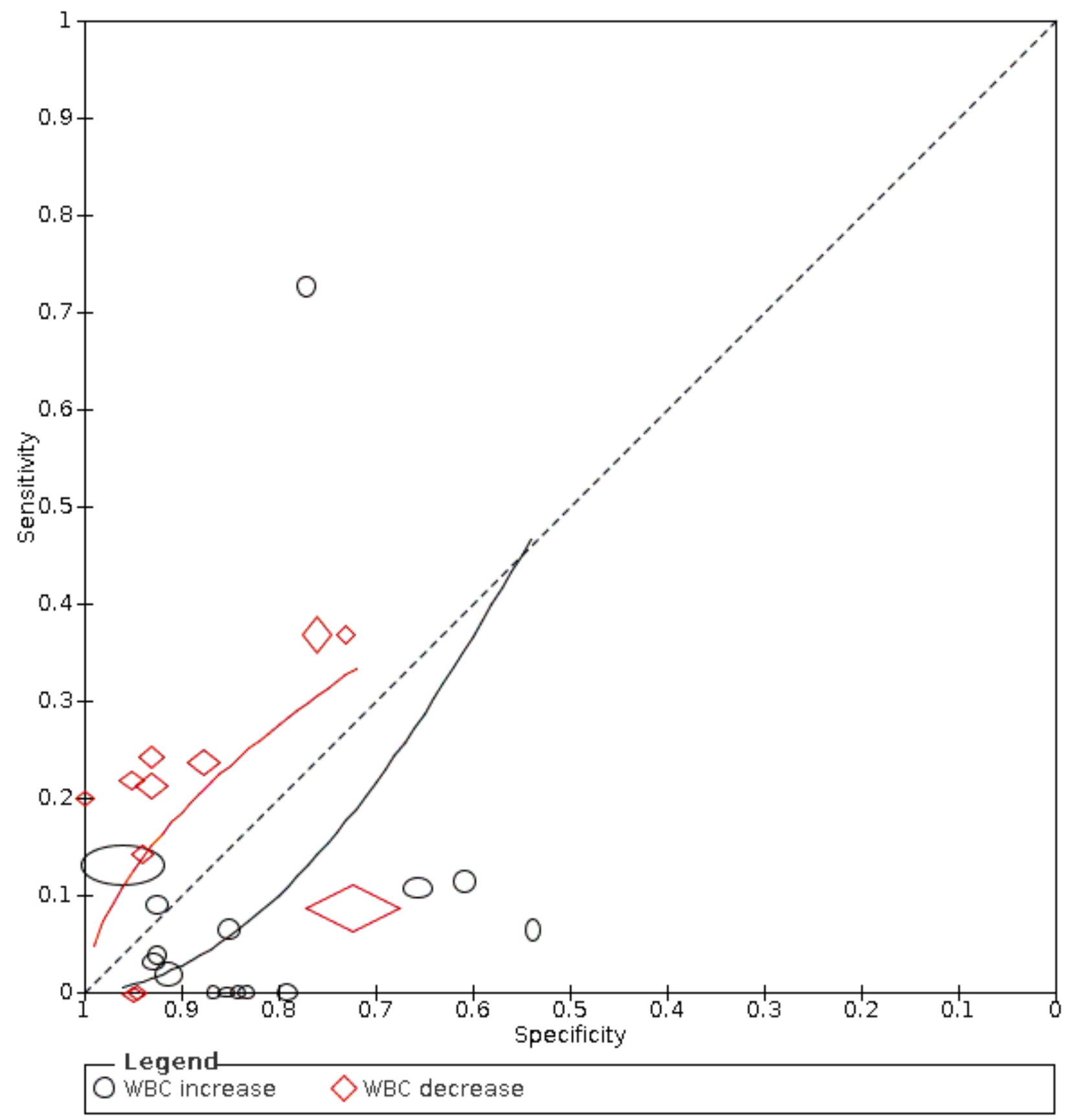

Sensitivity in the 15 included studies ranged from $0 \%$ to $73 \%$. Fourteen studies had a sensitivity within the range between $0 \%$ and $13 \%$ and one study reported a sensitivity of $73 \%$. This outlier also was the only study that used the lower cut-off of $6.4 \times 10^{9}$ cells/L. Specificity ranged from $54 \%$ to $96 \%$.

The median specificity was $85 \%$, with the interquartile range from $78 \%(\mathrm{Q} 1)$ to $92 \%(\mathrm{Q} 3)$. The summary estimate of sensitivity following from the HSROC model and corresponding with a specificity of $78 \%$, was $12 \%(95 \% \mathrm{Cl} 4 \%$ to $31 \%)$. The summary estimate of sensitivity corresponding with the median specificity of $85 \%$, was $6 \%(95 \% \mathrm{Cl} 2 \%$ to $17 \%)$ and the summary estimate of sensitivity corresponding with a specificity of $92 \%$, was $2 \%(95 \% \mathrm{Cl}$ $0 \%$ to $8 \%)$

\section{White blood cell count decrease}

Eleven studies (1211 cases/3900 non-cases) reported on white blood cell count decrease (Figure 3). The cut-off values for a decrease in white blood cell count varied from $3.5 \times 10^{9}$ cells $/ L$ to 4.0 $x 10^{9}$ cells/L. The median prevalence of COVID-19 in the nine single- 
gate studies was $28 \%$ (IQR $20 \%$ to $47 \%$ ). Sensitivity in the 11 studies ranged from $0 \%$ to $37 \%$. Specificity ranged from $72 \%$ to $100 \%$.

The median specificity was $93 \%$, with the interquartile range from $82 \%(\mathrm{Q} 1)$ to $95 \%(\mathrm{Q} 3)$. The summary estimates of sensitivity corresponding to these numbers were: $26 \%(95 \% \mathrm{Cl} 15 \%$ to $40 \%)$ at a specificity of $82 \% ; 25 \%(95 \% \mathrm{Cl} 8 \%$ to $27 \%)$ at a specificity of $93 \%$; and $22 \%(95 \% \mathrm{Cl} 5 \%$ to $26 \%)$ at a specificity of $95 \%$.

\section{Neutrophil count increase}

Eleven studies (824 cases/1014 non-cases) reported on neutrophil count (Figure 4). The cut-off values for an increase in neutrophil count varied from $6.3 \times 10^{9}$ cells/L to $7.0 \times 10^{9}$ cells/L, with the exception of one study that used a cut-off value of $4.6 \times 10^{9}$ cells $/ L$. The median prevalence of COVID-19 in the eight single-gate studies was $36 \%$ (IQR $25 \%$ to $61 \%$ ).

Figure 4. Summary ROC plot of tests: neutrophil count increase, and neutrophil count decrease

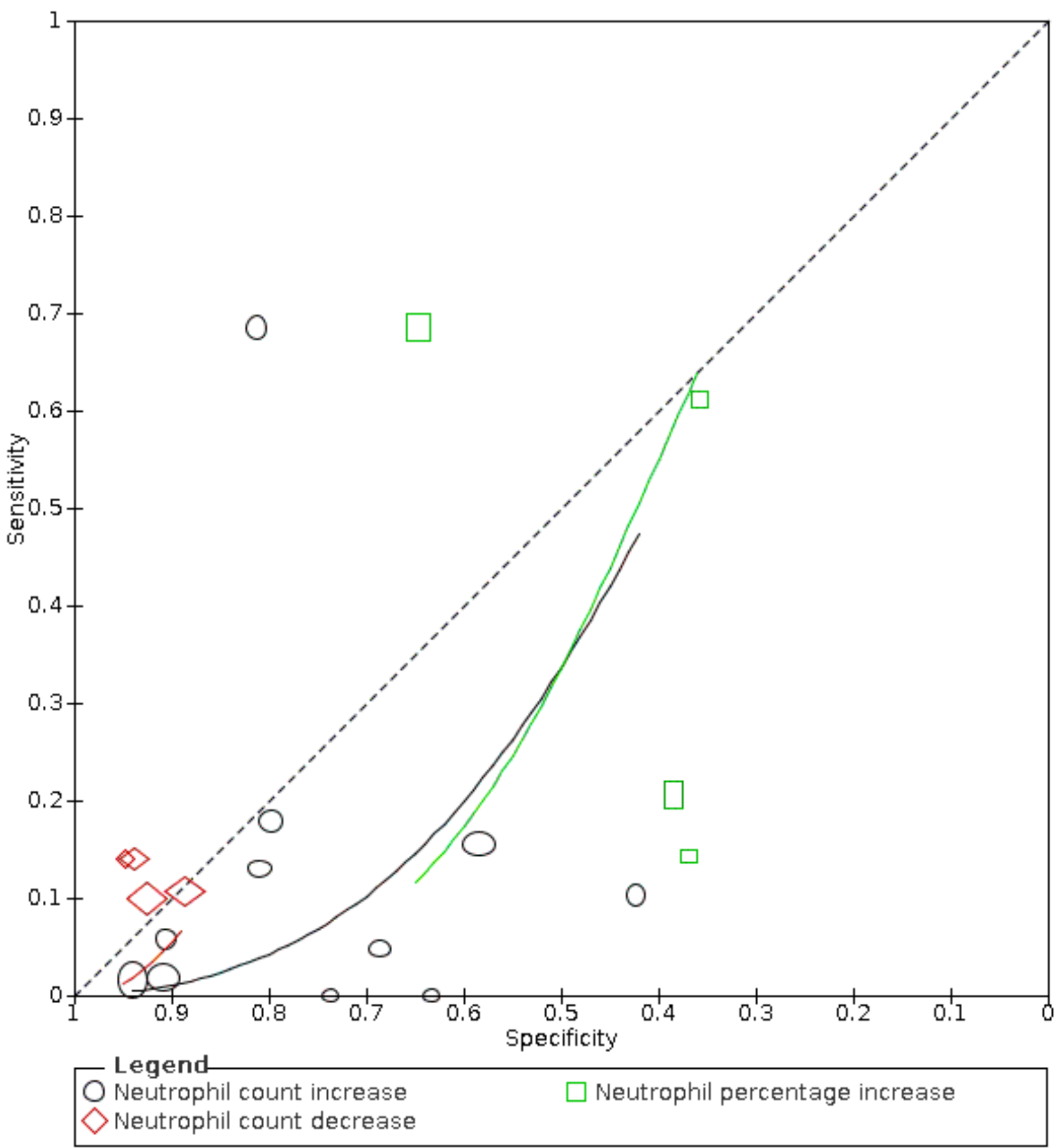

Routine laboratory testing to determine if a patient has COVID-19 (Review) 
Sensitivity ranged from $0 \%$ to $68 \%$; in 10 studies the sensitivity ranged between $0 \%$ and $18 \%$, one study reported a sensitivity of $68 \%$ (this outlier is probably due to the low cut-off value of $4.6 \times 10^{9}$ cells/L). Specificity ranged from $42 \%$ to $94 \%$, with a median of $80 \%$ (IQR 66\% to $86 \%$ ).

Meta-analysis yielded a sensitivity of $13 \%(95 \% \mathrm{Cl} 4 \%$ to $38 \%), 4 \%$ (95\% $\mathrm{Cl} 1 \%$ to $17 \%$ ) and $2 \%(95 \% \mathrm{Cl} 0 \%$ to $12 \%$ ) at fixed specificity of $66 \%$ (Q1), $80 \%$ (median) and $86 \%$ (Q3), respectively.

\section{Neutrophil count decrease}

Four studies (220 cases/514 non-cases) reported on the accuracy of decrease in neutrophil count (Figure 4). The cut-off values for a decrease in neutrophil count varied from $1.8^{\star} 10^{9}$ cells/L to $2^{\star} 10^{9}$ cells/L. The median prevalence of COVID-19 in the three singlegate studies was $27 \%$ (IQR $34 \%$ to $24 \%$ ). The sensitivity of the four studies ranged from $10 \%$ to $14 \%$ and specificity ranged from $89 \%$ to $95 \%$. Meta-analysis yielded a sensitivity of $12 \%$ (95\% Cl $1 \%$ to $54 \%), 10 \%(95 \% \mathrm{Cl} 1 \%$ to $56 \%)$ and $8 \%(95 \% \mathrm{Cl} 1 \%$ to $54 \%)$ at a fixed specificity of 92\% (Q1), 93\% (median) and 94\% (Q3), respectively.

\section{Neutrophil percentage increase}

Four studies (176 cases/107 non-cases) reported on the accuracy of increase in neutrophil percentage (Figure 4). The cut-off values for an increase in neutrophil count varied from $65.78 \%$ to $75.0 \%$. The median prevalence of COVID-19 in the three single-gate studies was $67 \%$ (IQR $39 \%$ to $74 \%$ ). The sensitivity of the four studies ranged from $14 \%$ to $68 \%$ and specificity ranged from $36 \%$ to $65 \%$. Metaanalysis yielded a sensitivity of $62 \%(95 \% \mathrm{Cl} 1 \%$ to $100 \%), 59 \%(95 \%$ $\mathrm{Cl} 1 \%$ to $100 \%)$ and $44 \%(95 \% \mathrm{Cl} 1 \%$ to $99 \%)$ at fixed specificity of 37\% (Q1), 38\% (median) and 45\% (Q3), respectively.

\section{Monocyte count increase}

Four studies (126 cases/332 non-cases) reported on monocyte increase (Figure 5). The cut-off values for an increase in monocyte count varied from 0.00 cells/L to 0.8 cells/L. The median prevalence of COVID-19 in the two single-gate studies was $73 \%$. Sensitivity ranged from $10 \%$ to $14 \%$; Specificity ranged from $56 \%$ to $89 \%$. Metaanalysis yielded a sensitivity of $14 \%(95 \% \mathrm{Cl} 6 \%$ to $30 \%), 13 \%$ (95\% $\mathrm{Cl} 6 \%$ to $26 \%)$ and $12 \%(95 \% \mathrm{Cl} 7 \%$ to $20 \%)$ at fixed specificity of $67 \%(\mathrm{Q} 1), 73 \%$ (median) and $80 \%(\mathrm{Q} 3)$, respectively. 
Figure 5. Summary ROC plot of monocyte count increase

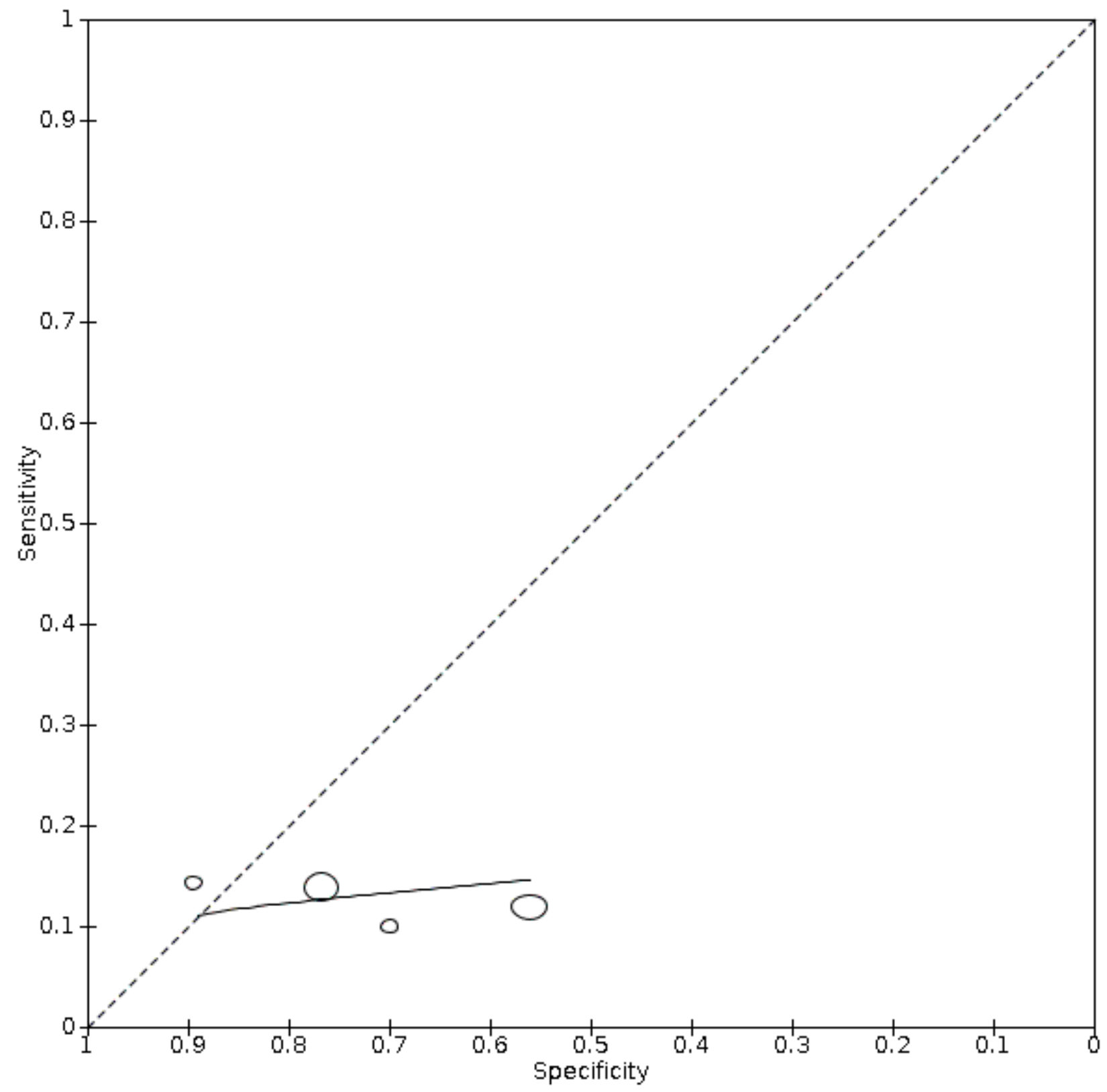

\section{Lymphocyte count decrease}

Thirteen studies (2752 cases/1066 non-cases) reported on decrease in lymphocyte count (Figure 6). The cut-off values for a decrease in lymphocyte count ranged from $8.0^{\star} 10^{9}$ cells/L to $1.1^{\star} 10^{9}$ cells/ L. The median prevalence of COVID-19 in the 11 single-gate studies was $37 \%$ ( $27 \%$ to $65 \%$ ), with sensitivity ranging from $0 \%$ to $81 \%$, with one outlier of $0 \%$ (based on two COVID-19 cases and specificity from $33 \%$ to $89 \%$. Meta-analysis yielded a sensitivity of $100 \%$ (95\% $\mathrm{Cl} 81 \%$ to $100 \%), 64 \%(95 \% \mathrm{Cl} 28 \%$ to $89 \%)$ and $0 \%(95 \% \mathrm{Cl} 0 \%$ to $24 \%$ ) at fixed specificity of $43 \%$ (Q1), $53 \%$ (median) and $71 \%(\mathrm{Q} 3)$, respectively. 
Figure 6. Summary ROC plot of lymphocyte count decrease

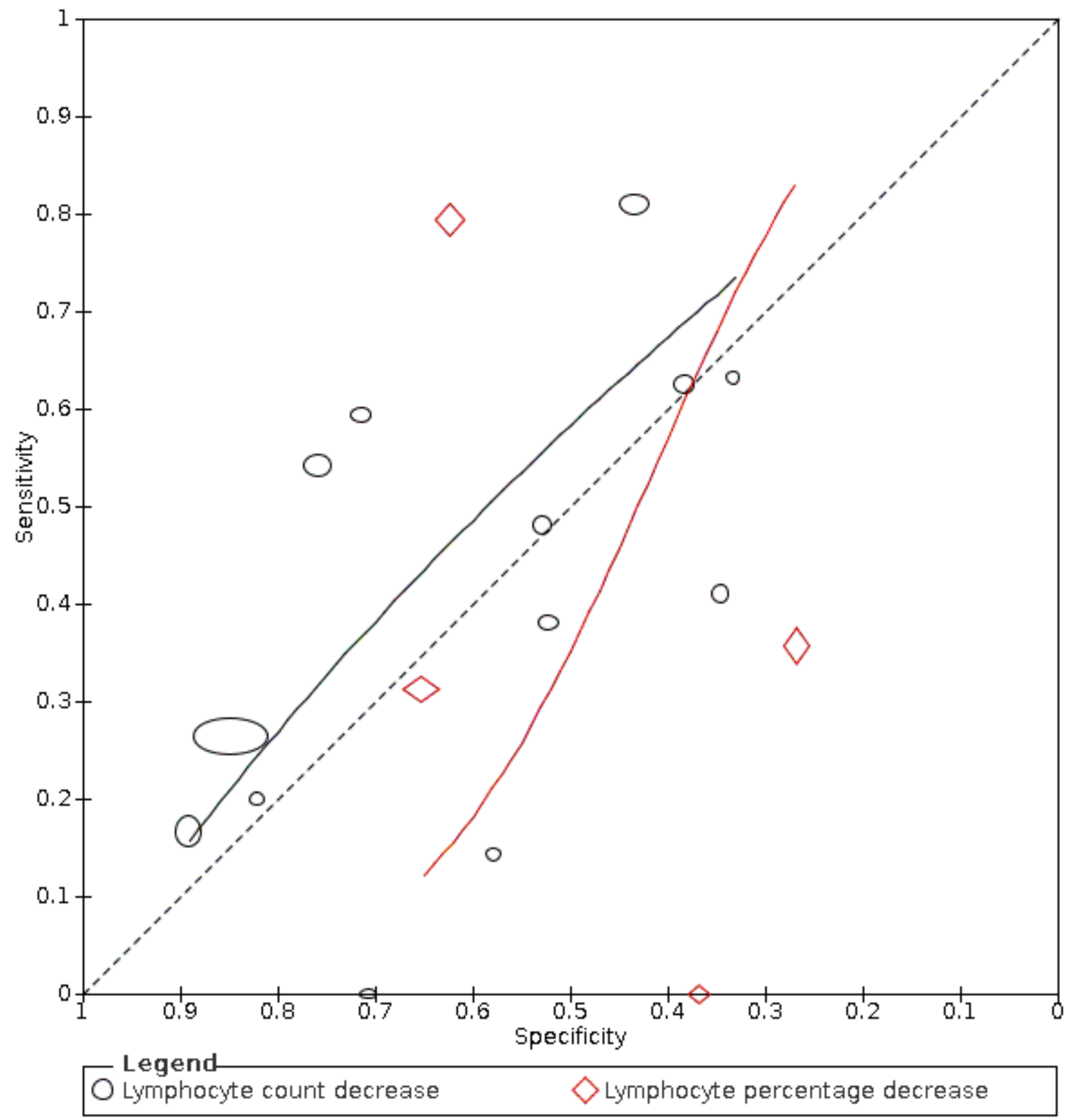

\section{Lymphocyte percentage decrease}

Four studies (190 cases/177 non-cases) reported on decrease in lymphocyte percentage (Figure 6). The cut-off values for a decrease in lymphocyte percentage ranged from $20 \%$ to $23.65 \%$. The median prevalence of COVID-19 in the 11 single-gate studies was $37 \%(27 \%$ to $65 \%$ ), with sensitivity ranging from $0 \%$ to $79 \%$ and specificity from $27 \%$ to $65 \%$. Meta-analysis yielded a sensitivity of $70 \%(95 \%$ $\mathrm{Cl} 0 \%$ to $100 \%), 35 \%(95 \% \mathrm{Cl} 0 \%$ to $99 \%)$ and $14 \%(95 \% \mathrm{Cl} 0 \%$ to $99 \%$ ) at fixed specificity of 34\% (Q1), 50\% (median) and 63\% (Q3), respectively.

\section{Platelets decrease}

Four studies (939 cases/3232 non-cases) reported on decrease in platelets (Figure 7). The cut-off values for a decrease in platelets ranged from 0.00 to 300.0 per microlitre. The median prevalence of COVID-19 in the three single-gate studies was $76 \%$ (38\% to $87 \%$ ), with sensitivity ranging from $13 \%$ to $30 \%$ and specificity from $71 \%$ to $100 \%$. Meta-analysis yielded a sensitivity of $23 \%(95 \% \mathrm{Cl} 13 \%$ to $38 \%), 19 \%(95 \% \mathrm{Cl} 10 \%$ to $32 \%)$ and $16 \%(95 \% \mathrm{Cl} 7 \%$ to $31 \%)$ at fixed specificity of $83 \%$ (Q1), $88 \%$ (median) and $92 \%$ (Q3), respectively. 
Figure 7. Summary ROC plot of 22 platelets, decreased

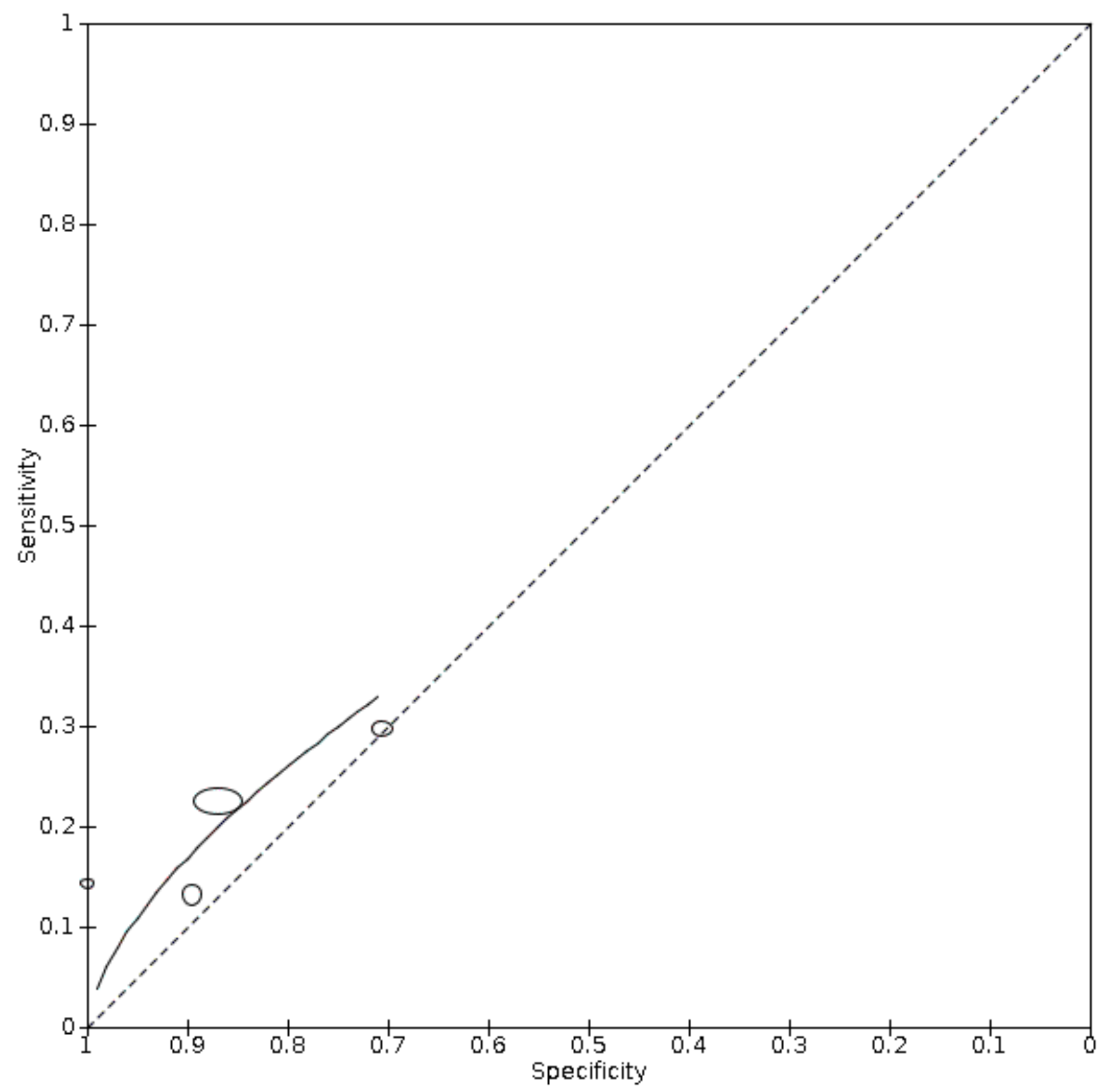

\section{Liver function tests}

\section{Alanine aminotransferase (ALT) increase}

Nine studies (1375 cases/3787 non-cases) reported on ALT increase (Figure 8). The cut-off values for in ALT increase varied from $40 \mathrm{U} / \mathrm{L}$ to $50 \mathrm{U} / \mathrm{L}$. The median prevalence of COVID-19 in the seven single- gate studies was $42 \%$ (IQR $34 \%$ to $66 \%$ ). Sensitivity ranged from $10 \%$ to $28 \%$ and specificity ranged from $74 \%$ to $100 \%$. Meta-analysis yielded a sensitivity of $23 \%(95 \% \mathrm{Cl} 14 \%$ to $35 \%), 12 \%$ (95\% Cl $3 \%$ to $34 \%)$ and $4 \%(95 \% \mathrm{Cl} 0 \%$ to $41 \%)$ at fixed specificity of $85 \%(\mathrm{Q} 1)$, 92\% (median) and $97 \%(\mathrm{Q} 3)$, respectively. 
Figure 8. Summary ROC plot of tests: alanine aminotransferase (ALT) increase, aspartate aminotransferase( AST) increase.

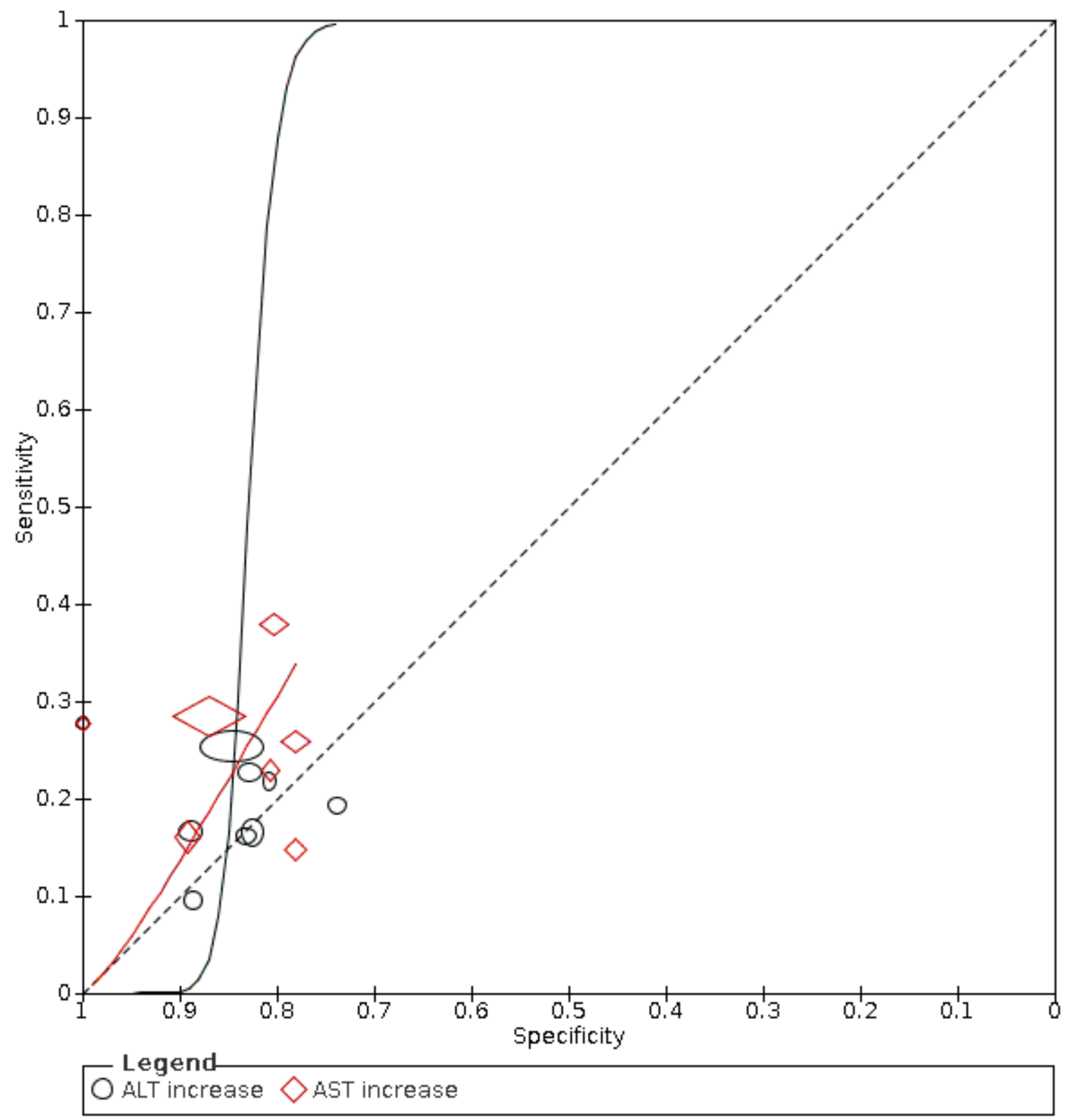

\section{Aspartate aminotransferase (AST) increase}

Seven studies (1260 cases/3631 non-cases) reported on AST increase (Figure 8). The cut-off values of AST increase varied from $35 \mathrm{U} / \mathrm{L}$ to $40 \mathrm{U} / \mathrm{L}$. The median prevalence of COVID-19 in the six single-gate studies was 53\% (IQR $29 \%$ to $68 \%$ ). Sensitivity ranged from $15 \%$ to $38 \%$, and specificity from $78 \%$ to $100 \%$. Meta-analysis yielded a sensitivity of $32 \%(95 \% \mathrm{Cl} 17 \%$ to $52 \%), 29 \%$ (95\% Cl 17\% to $45 \%)$ and $17 \%(95 \% \mathrm{Cl} 8 \%$ to $33 \%)$ at fixed specificity of $79 \%(\mathrm{Q} 1)$, $81 \%$ (median) and $88 \%(\mathrm{Q} 3)$, respectively.

\section{Albumin decrease}

Four studies (799 cases/3273 non-cases) reported on albumin decrease (Figure 9). The cut-off values of albumin decrease varied from 0 to $3.5 \mathrm{~g} / \mathrm{L}$. The median prevalence of COVID-19 in the three single-gate studies was $75 \%$ (IQR $51 \%$ to $87 \%$ ). Sensitivity ranged from $4 \%$ to $55 \%$, and specificity from $16 \%$ to $87 \%$. Meta-analysis yielded a sensitivity of $36 \%(95 \% \mathrm{Cl} 7 \%$ to $82 \%), 21 \%(95 \% \mathrm{Cl} 3 \%$ to $67 \%)$ and $13 \%(95 \% \mathrm{Cl} 1 \%$ to $64 \%)$ at fixed specificity of $46 \%(\mathrm{Q} 1)$, $66 \%$ (median) and 79\% (Q3), respectively. 
Figure 9. Summary ROC plot of tests: 30 total bilirubin (TBIL) increase, 36 albumin (ALB) decrease

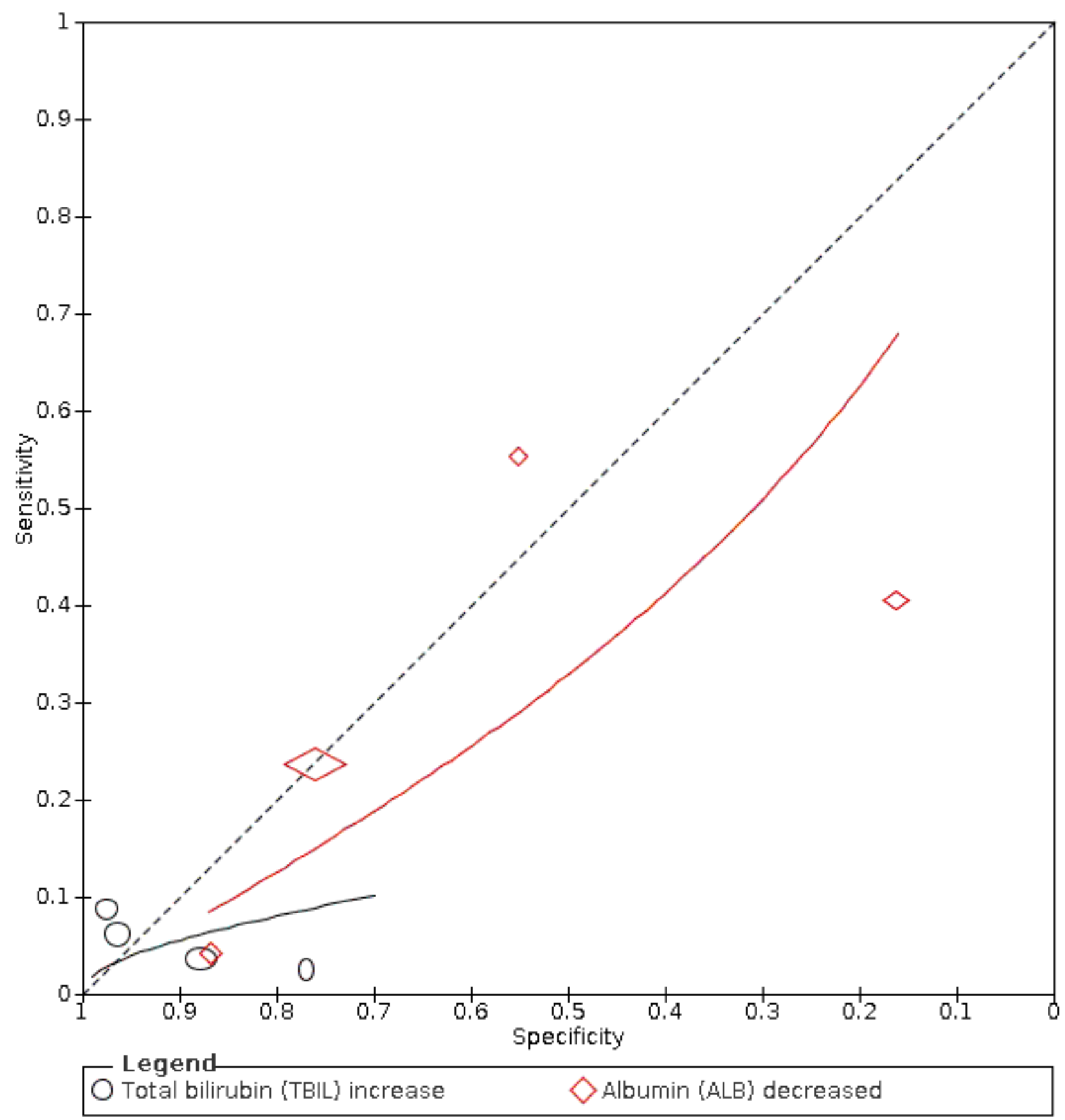

\section{Total bilirubin increase}

Four studies (333 cases/438 non-cases) reported total bilirubin increase (Figure 9). The cut-off varied from 0 to $21 \mu \mathrm{mol} / \mathrm{L}$. The median prevalence of COVID-19 in the four single-gate studies was $51 \%$ (IQR $25 \%$ to $61 \%$ ). Sensitivity ranged from $3 \%$ to $9 \%$ and specificity ranged from $77 \%$ to $97 \%$. Meta-analysis yielded a sensitivity of $23 \%$ ( $95 \% \mathrm{Cl} 14 \%$ to $35 \%$ ), $12 \%$ (95\% Cl $3 \%$ to $34 \%$ ) and $4 \%(95 \% \mathrm{Cl} 0 \%$ to $41 \%)$ at fixed specificity of $85 \%$ (Q1), $92 \%$ (median) and $97 \%(\mathrm{Q} 3)$, respectively.

\section{Markers of inflammation}

\section{C-reactive protein (CRP) increase}

Fourteen studies (997 cases/1284 non-cases) reported on CRP increase (Figure 10). The cut-off values for an increase in CRP increase varied from $8 \mathrm{mg} / \mathrm{L}$ to $34.8 \mathrm{mg} / \mathrm{L}$. The median prevalence of COVID-19 in the 11 single-gate studies was $51 \%$ (IQR $28 \%$ to $60 \%$ ). Sensitivity ranged from $0 \%$ to $95 \%$, with one outlier of $0 \%$ (based on two COVID-19 cases), and the other 13 studies ranging from $31 \%$ to $95 \%$. Specificity ranged from $20 \%$ to $81 \%$. Meta-analysis yielded

Routine laboratory testing to determine if a patient has COVID-19 (Review) 30

Copyright ( 2020 The Authors. Cochrane Database of Systematic Reviews published by John Wiley \& Sons, Ltd. on behalf of The Cochrane Collaboration. 
Figure 10. Summary ROC plot of tests: CRP increase and procalcitonin (PCT) increase

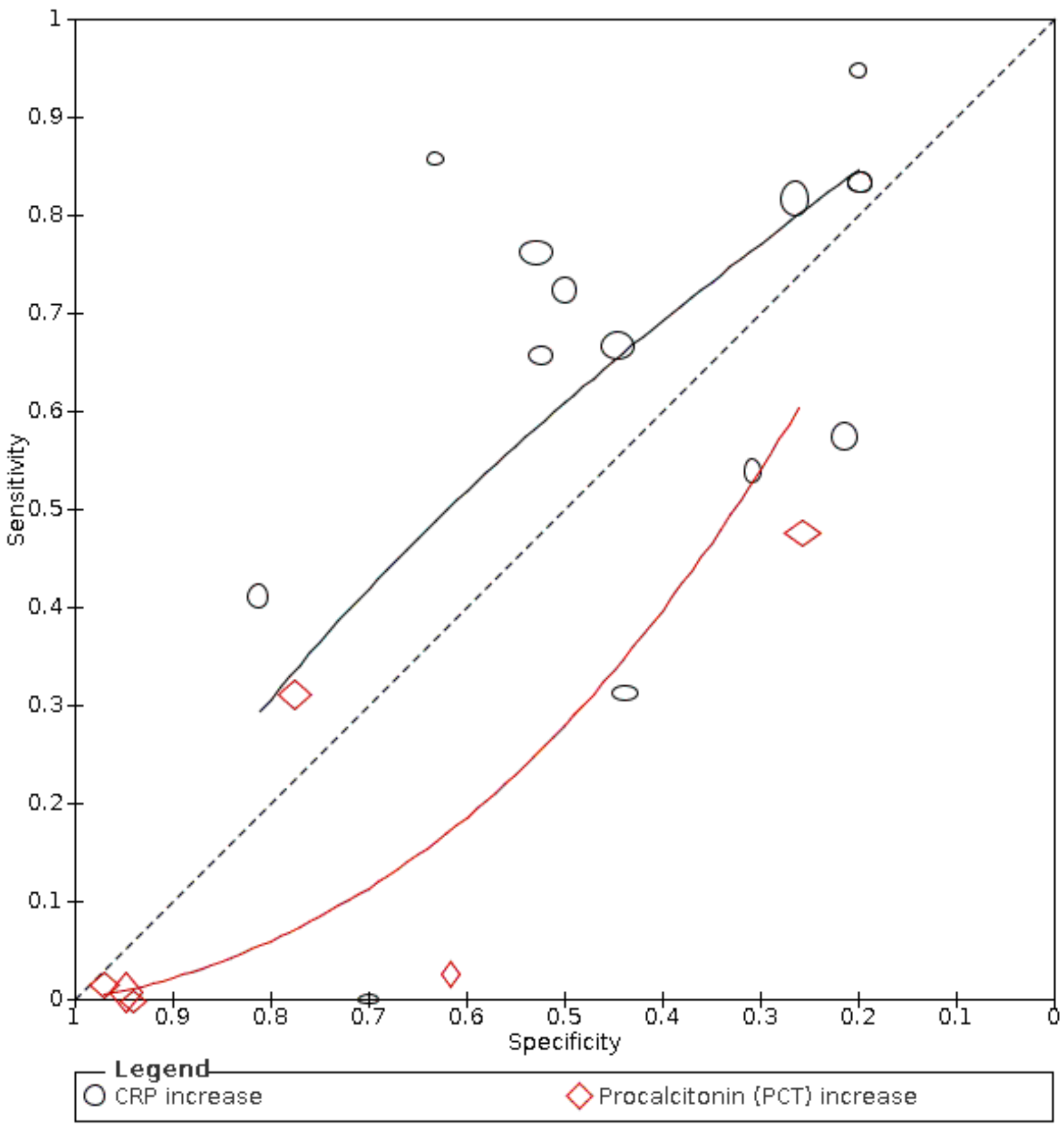

\section{Procalcitonin increase}

Six studies (607 cases/738 non-cases) reported on procalcitonin increase (Figure 10). The cut-off values for an increase in procalcitonin varied from $0.1 \mathrm{ng} / \mathrm{mL}$ to $0.5 \mathrm{ng} / \mathrm{mL}$. The median prevalence of COVID-19 in the five studies was 38\% (IQR 31\% to $70 \%)$. Sensitivity ranged from $0 \%$ to $48 \%$. Specificity ranged from $26 \%$ to $95 \%$. Meta-analysis yielded a sensitivity of $14 \%$ (95\% Cl $3 \%$ to $48 \%), 3 \%(95 \% \mathrm{Cl} 1 \%$ to $19 \%)$ and $1 \%(95 \% \mathrm{Cl} 0 \%$ to $10 \%)$ at fixed specificity of $66 \%(\mathrm{Q} 1), 86 \%$ (median) and $95 \%$ (Q3), respectively.

\section{IL-6 increase}

Four studies (86 cases/130 non-cases) reported on IL-6 increase (Figure 11). The cut-off values for an increase in IL-6 varied from 0 to $7 \mathrm{pg} / \mathrm{mL}$. The median prevalence of COVID-19 in the four 
studies was $84 \%$ (IQR $65 \%$ to $94 \%$ ). Sensitivity ranged from $22 \%$ to $86 \%$. Specificity ranged from $27 \%$ to $92 \%$. Meta-analysis yielded a sensitivity of $83 \%$ (95\% Cl $47 \%$ to $96 \%$ ), $73 \%$ (95\% Cl 36\% to $93 \%$ ) and $59 \%$ (95\% Cl $25 \%$ to $86 \%$ ) fixed specificity of $42 \%$ (Q1), $58 \%$ (median) and $74 \%(\mathrm{Q} 3)$, respectively.

Figure 11. Summary ROC plot of 53 interleukin-6 (IL-6) increase. Height and width of the symbols represent the number of cases and non-cases in the studies

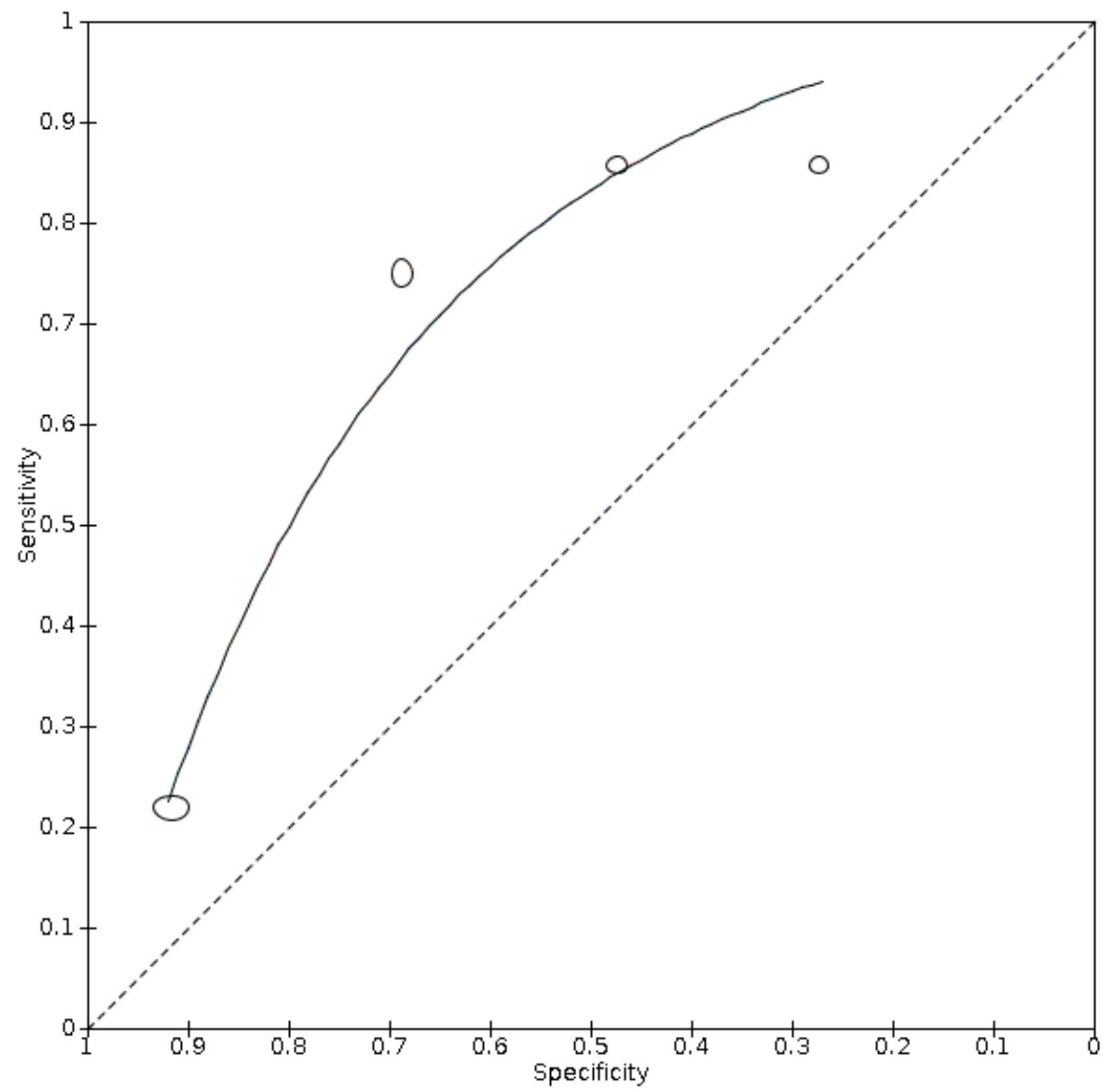

\section{Other tests}

\section{Creatine kinase increase}

Creatine kinase is a muscle damage marker, which increases upon muscle damage. It is sometimes used as an indicator for cardiac infarction. Five studies (575 cases/498 non-cases) reported on creatine kinase increase (Figure 12). The cut-off values for an increase in creatine kinase were between $174 \mu \mathrm{mol} / \mathrm{L}$ and $310 \mu \mathrm{mol} /$ $\mathrm{L}$. The median prevalence of COVID-19 in the five single-gate studies was $55 \%$ (IQR $37 \%$ to $70 \%$ ). Meta-analysis yielded a sensitivity of $15 \%$ (95\% Cl $10 \%$ to $22 \%$ ), $11 \%$ (95\% Cl $6 \%$ to $19 \%$ ) and $7 \%(95 \% \mathrm{Cl}$ $2 \%$ to $20 \%$ ) at fixed specificity of $88 \%$ (Q1), $94 \%$ (median) and $98 \%$ (Q3), respectively. 
Figure 12. Summary ROC plot of tests: 24 Serum creatinine increased, 25 Creatine kinase - increase, 55 lactate dehydrogenase (LDH) increase

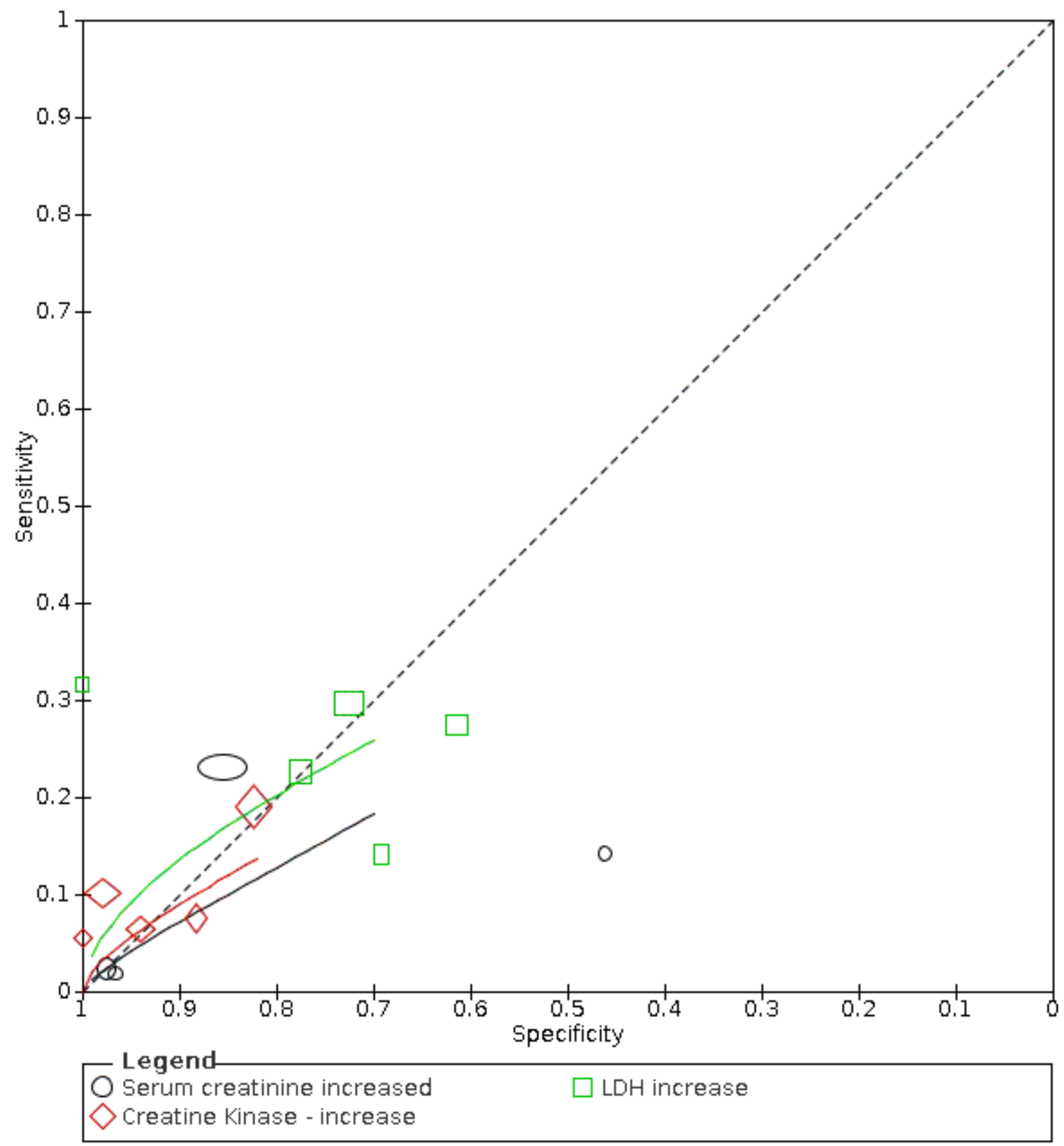

\section{Serum creatinine}

Serum creatinine is an indicator of kidney damage. Four studies (1005 cases/3311 non-cases), all single-gate design, reported on serum creatinine increase (Figure 12). The cut-off values for an increase in serum creatinine kinase were between $73 \mu \mathrm{mol} / \mathrm{L}$ and $133 \mathrm{\mu mol} / \mathrm{L}$. The prevalence in the four studies was $16 \%, 66 \%, 38 \%$ and $75 \%$. Meta-analysis yielded a sensitivity of $15 \%$ (95\% $\mathrm{Cl} 2 \%$ to
$63 \%$ ), $7 \%(95 \% \mathrm{Cl} 1 \%$ to $37 \%$ ) and $3 \%(95 \% \mathrm{Cl} 0 \%$ to $36 \%$ ) at fixed specificity of $76 \%$ (Q1), 91\% (median) and $97 \%(\mathrm{Q} 3)$, respectively.

\section{Lactate dehydrogenase (LDH) increase}

LDH is a general marker for tissue damage. Five studies (382 cases/431 non-cases) reported on LDH increase (Figure 12). The cutoff values for in LDH increase varied from 243 to $25 \mathrm{U} / \mathrm{L}$. The median prevalence of COVID-19 in the five single-gate studies was 54\% (IQR 
$40 \%$ to $71 \%$ ). Sensitivity ranged from $14 \%$ to $32 \%$ and specificity ranged from $61 \%$ to $100 \%$. Meta-analysis yielded a sensitivity of $26 \%$ (95\% Cl $15 \%$ to $42 \%$ ), $25 \%$ (95\% Cl $15 \%$ to $38 \%$ ) and $22 \%$ (95\% $\mathrm{Cl} 11 \%$ to $40 \%$ ) at fixed specificity of $69 \%$ (Q1), $72 \%$ (median) and $77 \%$ (Q3), respectively.

\section{Comparisons between tests}

For three tests, we found a pair of sensitivity and specificity where both sensitivity and specificity exceeded $50 \%$. These were IL-6 increase, CRP increase and lymphocyte count decrease. Using all available studies in an indirect comparison (i.e. unrestricted to head-to-head studies), we compared the test performance of IL-6 increase (4 studies), CRP increase (14 studies) and lymphocyte count decrease (13 studies) in one meta-regression analysis. The shape of the SROC curves significantly differed $(P<0.001)$. Figure 13 shows the summary ROC curves for the three tests in one Figure (Summary of findings 2). 
Figure 13. Summary ROC plot of tests: 12 lymphocyte count decrease, 32 CRP increase, 47 interleukin-6 (IL-6) increase

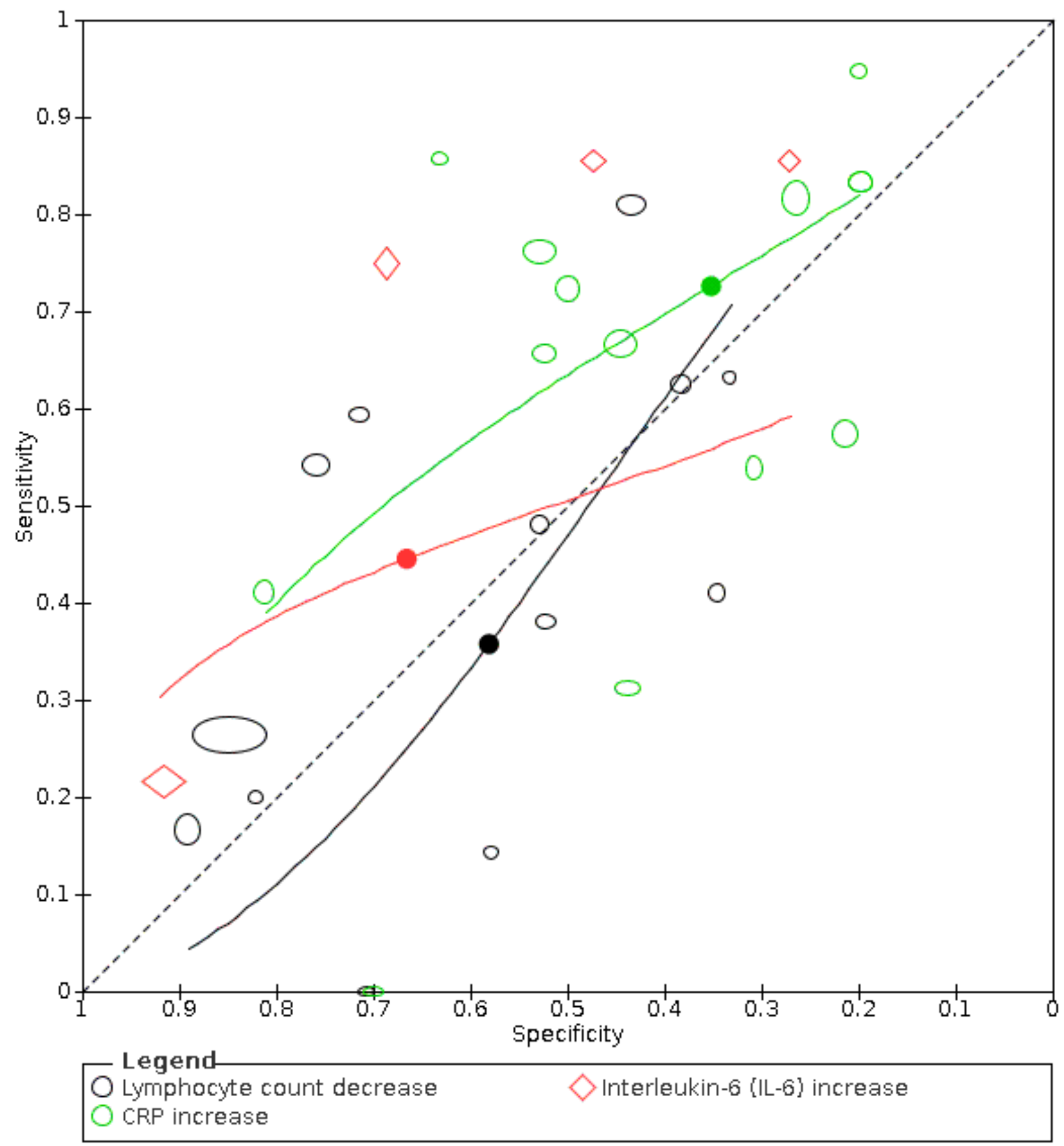

The median specificity in the 19 studies evaluating one or more of the three tests, was $52 \%$ (IQR $34 \%$ to $67 \%$ ). Within the specificity interquartile range, sensitivity varied between $6 \%(95 \% \mathrm{Cl} 0 \%$ to $49 \%)$ and $100 \%(22 \%$ to $100 \%)$ for lymphocyte count decrease, between $51 \%(95 \% \mathrm{Cl} 34 \%$ to $68 \%)$ and $73 \%(95 \% \mathrm{Cl} 64 \%$ to $80 \%)$ for CRP increase, and between $67 \%(95 \% \mathrm{Cl} 51 \%$ to $79 \%)$ and $73 \%$ (95\% $\mathrm{Cl} 45 \%$ to $79 \%$ ) for IL-6 increase.
Nine studies directly compared CRP increase with lymphocyte count decrease for the detection of COVID-19. Especially for lymphocyte count decrease, this direct comparison (Figure 14), shows a different picture from the indirect comparisons (Figure 13), or the separate analyses (Figure 6). Despite differences in cut-offs, the results from most studies were consistent with CRP increase showing higher sensitivity than the lymphocyte count decrease. The RDOR was $2.02(95 \% \mathrm{Cl} 1.47$ to 2.78$)$, meaning that the 
Figure 14. Summary ROC plot of tests: 12 lymphocyte count decrease, 32 CRP increase

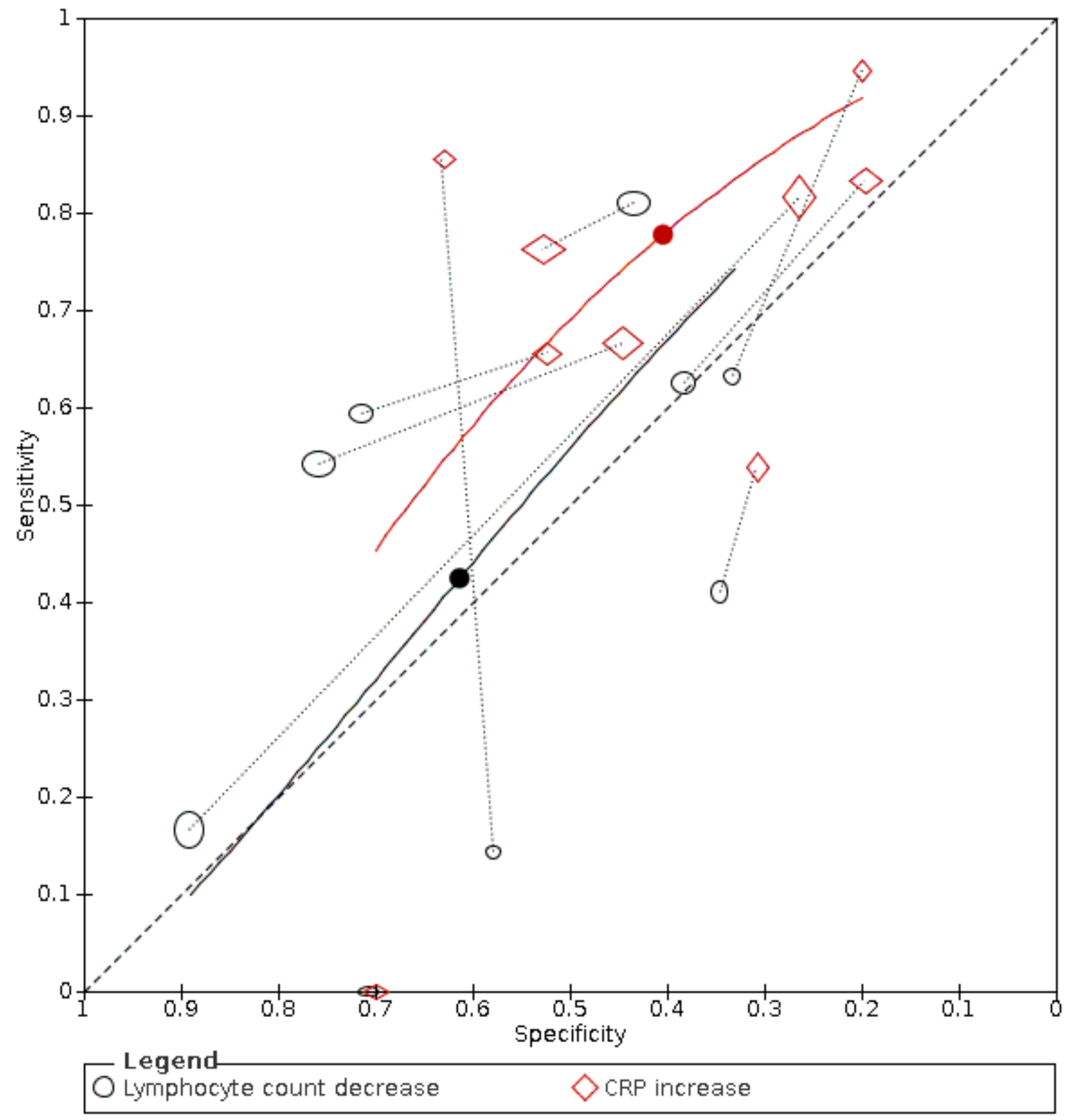

\section{DISCUSSION}

\section{Summary of main results}

We included 21 studies in this review and analyzed the results for 67 different routine laboratory tests, focusing on diagnosing COVID-19. For 16 tests, we have summarized the results in a meta-analysis. As the majority of the included studies only reported RT-PCR as a reference standard, the meta-analyses may be more applicable to detecting SARS-CoV-2 infection than COVID-19 diseased. Only three tests performed at sensitivity-specificity combinations where both sensitivity and specificity were above $50 \%$. There was low to very low certainty in the summary estimates of the tests.

The low accuracy of these tests does not render them useless. They are all indicators of the general health status of a patient. 
They may indicate infection, inflammation, or tissue damage and thus support diagnoses made based on other diseases. However, evidence to date suggests that in sick hospitalized patients, they cannot discriminate between COVID-19 and other diseases as the cause of infection, inflammation or tissue damage and should preferably not be used as stand-alone tests for COVID-19. As a triage test would require a high sensitivity $(<80 \%)$, these tests have limited use as triage tests. How these tests would perform in those with milder symptoms cannot be inferred from our data.

In some situations, where resources are very limited, these tests are the only ones at hand when making a diagnosis. In these situations, it may be worthwhile to consider the three tests with a slightly better performance than the others: lymphocyte count decrease, IL-6 increase and CRP increase. These tests are also available as point-of-care tests, although that is not how they were used in the included studies, so any inference should be made with caution.

Of those three, IL- 6 has the highest summary sensitivity at the highest median specificity. Both the median specificity and the boundary of the third quartile were above 50\% (58\% and $74 \%$ respectively). If we chose to use the test at a higher specificity of $74 \%$, then the sensitivity would only be $59 \%$ (95\% Cl $25 \%$ to $86 \%$ ). When testing 1000 people using this cut-off value, at $5 \%$ pre-test probability, then 29 or 30 out of 50 cases would have a true positive result and be contained or put in quarantine, and 20 or 21 out of 50 cases would be sent home, possibly infectious. It would also mean that of the 950 non-cases, 247 would be considered to be positive, while they are not. Using the test at a lower cut-off value to increase sensitivity, would decrease specificity even further.

The median pre-test probability of all included studies was $36 \%$ and most patients were hospitalized. In such a scenario, when testing 1000 people with IL-6 at a specificity of $74 \%$ and a sensitivity of $59 \%$, then 212 out of 360 cases would have a true positive result and be contained or put in quarantine, and 148 out of 360 cases would be sent home or to a non-COVID-19 ward, possibly infectious. It would also mean that of the 640 non-cases, 166 would be considered to be positive, while they are not.

Nine studies directly compared leukocyte count increase and CRP increase. From the meta-analysis including these two tests, we found that CRP is more accurate than leukocyte count increase, but as explained above, the point estimates do require caution when using the tests as sole markers. Furthermore, we did not assess the quality of the comparisons made in the included studies.

\section{Strengths and weaknesses of the review}

We assessed the diagnostic accuracy of a broad spectrum of routine laboratory tests for COVID-19. Included studies demonstrated considerable heterogeneity in the accuracy of many biomarkers, and used cut-off values and reference standards that were, in many cases, suboptimally described. The current review included a range of different cut-off values for most index tests, which we took into account using HSROC analyses and pooling studies with similar cutoff values for a given laboratory marker.

A limitation is suboptimal reporting that hampered assessment of the QUADAS-2 flow and timing domain in many studies. In many instances the timing of index test and reference standard was unclear, which could have led to unreliable results concerning the diagnostic abilities of the tests. While most studies used RT-
PCR as reference standard, some used a combination of RT-PCR and signs and symptoms or other tests. This potentially introduced heterogeneity because of differences in patients marked as cases and controls according to the differences in reference standards.

Some tests of interest, such as d-dimer or cardiac markers were evaluated in too few studies to meta-analyse their results.

\section{Applicability of findings to the review question}

We retrieved information on multiple index tests. The availability of laboratory tests is dependent on the type of hospital, department and available resources of the place in which the test is to be performed. In order to make the findings suitable for different settings we have included a broad range of biomarkers, and settings. We did not find studies that included participants in a primary care or general population setting. In clinical practice, not a single test, but the results of a combination of tests might be important for diagnosing COVID-19. These tests can be used for the first triage of patients in case of limited access to diagnostic tests, after which at a later stage further testing can be done. For triage tests, a high sensitivity is important to safely rule out the disease, however all tests had a low sensitivity. Also, the cut-off values used may differ by clinic and location, this could lead to different treatment decisions if a single patient were tested in different settings. In this review we included all different cut-off points available in current literature. Lastly, the reference standard in most studies was RT-PCR only, which means that there are concerns regarding applicability of the results of this review to COVID-19 as a target condition. However, the reporting of the studies was unclear and sometimes confusing. It may therefore be possible that in the study practice also other criteria were used to assess the diagnosis, but that this was not or insufficiently reported.

\section{AUTHORS' CONCLUSIONS}

\section{Implications for practice}

None of these markers as stand-alone tests are useful for accurately ruling in or ruling out COVID-19. As a triage test would require a high sensitivity $(<80 \%)$, these tests have limited value as triage tests. Although there is low or very low certainty about the summary estimates in this review, we do not expect that studies with a low risk of bias will show a better performance than the tests included.

\section{Implications for research}

Future studies focusing on the usefulness of routine laboratory tests for COVID-19 may consider a more representative sample of the population, focus on markers with prespecified, clinically sound cut-offs and focus on single, but also on the combination of regular blood markers. Furthermore, considering the test results as continuous values may be more informative, as larger deviations from the reference values will have greater impact on the health status of the tested people, and might enable more personalized treatment.

\section{ACK N O WLEDGEMENTS}

Members of the Cochrane COVID-19 Diagnostic Test Accuracy Review Group include: 
- the project team (Deeks JJ, Dinnes J, Takwoingi Y, Davenport C, Leeflang MMG, Spijker R, Hooft L, Van den Bruel A, McInnes MDF, Emperador D, Dittrich S, Cunningham J);

- the systematic review teams for each review:

* Molecular, antigen, and antibody tests (Adriano A, Beese S, Dretzke J, Ferrante di Ruffano L, Harris I, Price M, TaylorPhillips S)

* Signs and symptoms (Stuyf T, Domen J, Horn S)

* Routine laboratory markers (Yang B, Langendam M, Ochodo E, Guleid F, Holtman G, Verbakel J, Wang J, Stegeman I)

* Imaging tests (Salameh JP, McGrath TA, van der Pol CB, Frank RA, Prager R, Hare SS, Dennie C, Jenniskens K, Korevaar DA, Cohen JF, van de Wijgert J, Damen JAAG, Wang J);

- the wider team of systematic reviewers from University of Birmingham, UK who assisted with title and abstract screening across the entire suite of reviews for the diagnosis of COVID-19 (Agarwal R, Baldwin S, Berhane S, Herd C, Kristunas C, Quinn L, Scholefield B).

We thank Dr Jane Cunningham (World Health Organization) for participation in technical discussions and comments on the manuscript.

The Cochrane Editorial and Methods Department (EMD) Editorial Service collaborated with Cochrane Infectious Diseases Group (CIDG) on the management of the editorial process. We thank Helen Wakeford (EMD), Anne-Marie Stephani (EMD) and Deirdre
Walshe (CIDG) for editorial checks; Robin Featherstone and Douglas M Salzwedel for comments on the search; Jennifer Hilgart for comments on the abstract; to the peer referees for this review including Jessica Watson and Olabisi A. Oduwole; and Mike Brown and Paul Garner for sign-off comments. We thank Denise Mitchell for her efforts in copy-editing this review.

We would like to thank the Cochrane Diagnostic Test Accuracy (DTA) Editorial Team including Sophie Beese and Bella Harris for managing the editorial process; Karen Steingart for methods peer review, Matthew Grainge for providing statistical peer review and acting as Contact Editor for the full review, and to Prof Danielle Van der Windt who was the DTA Contact Editor for the protocol.

The editorial base of the Cochrane Infectious Diseases Group is funded by UK aid from the UK government for the benefit of lowand middle-income countries (project number 300342-104). The views expressed do not necessarily reflect the UK government's official policies.

Jonathan Deeks is a United Kingdom National Institute for Health Research (NIHR) Senior Investigator Emeritus. Yemisi Takwoingi is supported by a NIHR Postdoctoral Fellowship. Jonathan Deeks, Jacqueline Dinnes, Yemisi Takwoingi, and Clare Davenport are supported by the NIHR Birmingham Biomedical Research Centre. The views expressed are those of the authors and not necessarily those of the NHS, the NIHR, or the Department of Health and Social Care. 


\section{R E F E R E N C E S}

\section{References to studies included in this review}

Ai 2020b \{published data only\}

Ai J, Gong J, Xing L, He R, Tian F, Wang J, et al. Analysis of factors associated early diagnosis in coronavirus disease 2019 (COVID-19). medRxiv [Preprint] 2020. [DOI: doi.org/10.1101/2020.04.09.20059352]

\section{Chen 2020c \{published data only\}}

Chen X, Yang Y, Huang M, Liu L, Zhang X, Xu J, et al. Differences between COVID-19 and suspected then confirmed SARS-CoV-2negative pneumonia: a retrospective study from a single center. Journal of Medical Virology 2020 Apr 1 [Epub ahead of print]. [DOI: $10.1002 / j m v .25810]$

\section{Feng 2020 \{published data only\}}

Feng C, Huang Z, Wang L, Chen X, Zhai Y, Zhu F, et al. A novel triage tool of artificial intelligence assisted diagnosis aid system for suspected COVID-19 pneumonia in fever clinics. medRxiv [Preprint] 2020. [DOI: doi.org/10.1101/2020.03.19.20039099]

\section{Ferrari 2020 \{published data only\}}

Ferrari D, Motta A, Strollo M, Banfi G, Locatelli M. Routine blood tests as a potential diagnostic tool for COVID-19. Clinical Chemistry and Laboratory Medicine 2020;58(7):1095-9.

\section{Hsih 2020 \{published data only\}}

Hsih WH, Cheng MY, Ho MW, Chou CH, Lin PC, Chi CY, et al. Featuring COVID-19 cases via screening symptomatic patients with epidemiologic link during flu season in a medical center of central Taiwan. Journal of Microbiology, Immunology and Infection 2020;53(3):459-66.

\section{Li 2020d \{published data only\}}

Li H, Chen K, Liu M, Xu H, Xu Q. The profile of peripheral blood lymphocyte subsets and serum cytokines in children with 2019 novel coronavirus pneumonia. Journal of Infection 2020;8(1):115-20.

\section{Li 2020e \{published data only\}}

Li N, Han L, Peng M, Lv Y, Ouyang Y, Liu K, et al. Maternal and neonatal outcomes of pregnant women with COVID-19 pneumonia: a case-control study. Clinical Infectious Diseases 2020. [DOI: doi.org/10.1093/cid/ciaa352]

\section{Li $2020 f$ \{published data only\}}

Li Q, Ding X, Xia G, Geng Z, Chen F, Wang L, et al. A simple laboratory parameter facilitates early identification of COVID-19 patients. medRxiv [Preprint] 2020. [DOI: doi.org/10.1101/2020.02.13.20022830]

\section{Li 2020g \{published data only\}}

Li YX, Wu W, Yang T, Zhou W, Fu YM, Feng QM, et al. Characteristics of peripheral blood leukocyte differential counts in patients with COVID-19. Zhonghua Nei Ke Za Zhi 2020;59(0):E003

\section{Liang 2020 \{published data only\}}

Liang Y, Liang J, Zhou Q, Li X, Lin F, Deng Z, et al. Prevalence and clinical features of 2019 novel coronavirus disease (COVID-19) in the fever clinic of a teaching hospital in Beijing: a singlecenter, retrospective study. medRxiv [Preprint] 2020. [DOI: doi.org/10.1101/2020.02.25.20027763]

\section{Liu 2020 \{published data only\}}

Liu R, Ma Q, Han H, Su H, Liu F, Wu K, et al. The value of urine biochemical parameters in the prediction of the severity of coronavirus disease 2019. Clinical Chemistry and Laboratory Medicine 2020;58(7):1121-4.

\section{Lu 2020 \{published data only\}}

Lu J, Hu S, Fan R, Liu Z, Yin X, Wang Q, et al. ACP risk grade: a simple mortality index for patients with confirmed or suspected severe acute respiratory syndrome coronavirus 2 disease (COVID-19) during the early stage of outbreak in Wuhan, China. medRxiv [Preprint] 2020. [DOI: doi.org/10.1101/2020.02.20.20025510]

\section{Mardani 2020 \{published data only\}}

Mardani R, Ahmadi Vasmehjani A, Zali F, Gholami A, Mousavi Nasab SD, Kaghazian $\mathrm{H}$, et al. Laboratory parameters in detection of COVID-19 patients with positive RT-PCR; a diagnostic accuracy study. Archives of Academic Emergency Medicine 2020;8(1):e43.

\section{Miao 2020 \{published data only\}}

Miao C, Zhuang J, Jin M, Xiong H, Huang P, Zhao Q, et al. A comparative multi-centre study on the clinical and imaging features of comfirmed and uncomfirmed patients with COVID-19. medRxiv [Preprint] 2020. [DOI: doi.org/10.1101/2020.03.22.20040782]

\section{Pan 2020 \{published data only\}}

Pan Y, Ye G, Zeng X, Liu G, Zeng X, Jiang X, et al. Can routine laboratory tests discriminate SARS-CoV-2 infected pneumonia from other causes of community acquired pneumonia? Clinical and Translational Medicine 2020;10(1):161-8.

\section{Rentsch 2020 \{published data only\}}

Rentsch CT, Kidwai-Khan F, Tate JP, Park LS, King JT, Skanderson M, et al. COVID-19 testing, hospital admission, and intensive care among 2,026,227 United States veterans aged 54-75 years. medRxiv [Preprint] 2020. [DOI: $10.1101 / 2020.04 .09 .20059964]$

Yang 2020b \{published data only\} Yang L, Bai Y, Qiu Q, Wang T, Jiang L, Liu X, et al. Earlystage vigilance of novel coronavirus pneumonia. Available at ssrn.com/abstract=3544820 [Preprint] 2020. [DOI: dx.doi.org/10.2139/ssrn.3544820]

Yang 2020c \{published data only\}

Yang Z, Lin D, Chen X, Qiu J, Li S, Huang R, et al. Distinguishing COVID-19 from influenza pneumonia in the early stage through CT imaging and clinical features. medRxiv [Preprint] 2020. [DOI: doi.org/10.1101/2020.04.17.20061242] 
Zhang 2020 \{published data only\}

Zhang Y, Zheng L, Liu L, Zhao M, Xiao J, Zhao Q. Liver impairment in COVID-19 patients: a retrospective analysis of 115 cases from a single center in Wuhan city, China. Liver International 2020;40(9):2095-2103.

Zhao 2020 \{published data only\}

Zhao D, Yao F, Wang L, Zheng L, Gao Y, Ye J, et al. A comparative study on the clinical features of COVID-19 pneumonia to other pneumonias. Clinical Infectious Diseases 2020;71(15):756-61. [DOI: 10.1093/cid/ciaa247]

Zhu 2020 \{published data only\}

Zhu W, Xie K, Lu H, Xu L, Zhou S, Fang S. Initial clinical features of suspected coronavirus disease 2019 in two emergency departments outside of Hubei, China. Journal of Medical Virology 2020. [DOI: doi.org/10.1002/jmv.25763]

\section{References to studies excluded from this review}

Ai 2020a \{published data only\}

Ai JW, Zhang HC, Xu T, Wu J, Zhu M, Yu YQ et al. Optimizing diagnostic strategy for novel coronavirus pneumonia, a multicenter study in Eastern China. medRxiv [Preprint] 2020. [DOI: https://doi.org/10.1101/2020.02.13.20022673]

\section{Chen 2020a \{published data only\}}

Chen X, Ling J, Mo P, Zhang Y, Jiang Q, Ma Z, et al. Restoration of leukomonocyte counts is associated with viral clearance in COVID-19 hospitalized patients. medRxiv [Preprint] 2020. [DOI: https://doi.org/10.1101/2020.03.03.20030437]

\section{Chen 2020b \{published data only\}}

Chen Y, Chen L, Deng Q, Zhang G, Wu K, Ni L, et al. The presence of SARS-CoV-2 RNA in feces of COVID-19 patients. Journal of Medical Virology 2020;92(7). [DOI: https://doi.org/10.1002/ jmv.25825]

\section{Cheng 2020 \{published data only\}}

Cheng Z, Lu Y, Cao Q, Qin L, Pan Z, Yan F, et al. Clinical features and chest CT manifestations of coronavirus disease 2019 (COVID-19) in a single-center study in Shanghai, China. American Journal of Roentgenology 2020;215(1):121-6. [DOI: doi.org/10.2214/AJR.20.22959]

\section{Giamarellos 2020 \{published data only\}}

Giamarellos-Bourboulis EJ, Netea MG, Rovina N, Akinosoglou K, Antoniadou A, Antonakos N, et al. Complex immune dysregulation in COVID-19 patients with severe respiratory failure. Cell Host \& Microbe 2020;27(6):992-1000. [DOI: https:// doi.org/10.1016/j.chom.2020.04.009]

\section{Han 2020 \{published data only\}}

Han H, Yang L, Liu R, Liu F, Wu KL, Li J, et al. Prominent changes in blood coagulation of patients with SARS-CoV-2 infection. Clinical Chemistry and Laboratory Medicine 2020;58(7). [DOI: https://doi.org/10.1515/cclm-2020-0188]

\section{Kurstjens 2020 \{published data only\}}

Kurstjens S, van der Horst A, Herpers R, Geerits MW, Kluitersde Hingh YC, Göttgens EL, et al. Rapid identification of SARSCoV-2-infected patients at the emergency department using routine testing. medRxiv [Preprint] 2020. [DOI: https:// doi.org/10.1101/2020.04.20.20067512]

\section{Li 2020a \{published data only\}}

Li J, Li S, Cai Y, Liu Q, Li X, Zeng Z, et al. Epidemiological and clinical characteristics of 17 hospitalized patients with 2019 novel coronavirus Infections outside Wuhan, China. medRxiv [Preprint] 2020. [DOI: https:// doi.org/10.1101/2020.02.11.20022053]

\section{Li 2020b \{published data only\}}

Li Y, Wang Z, Hui Y, Tong X, Mao X, Huang L, et al. Clinical characteristics of 77 novel coronavirus 2019 infected patients with respiratory failure in the terminal stage in Wuhan. Available at ssrn.com/abstract $=3551325$ [Preprint] 2020. [DOI: dx.doi.org/10.2139/ssrn.3551325]

\section{Li 2020c \{published data only\}}

Li YY, Wang WN, Lei Y, Zhang B, Yang J, Hu JW, et al. Comparison of the clinical characteristics between RNA positive and negative patients clinically diagnosed with 2019 novel coronavirus pneumonia. Zhonghua Jie He He Hu Xi Za Zhi 2020;43(5):427-430. [DOI: 10.3760/ cma.j.cn112147-20200214-00095 ]

\section{Ling 2020 \{published data only\}}

Ling Y, Xu SB, Lin YX, Tian D, Zhu ZQ, Dai FH, et al. Persistence and clearance of viral RNA in 2019 novel coronavirus disease rehabilitation patients. Chinese Medical Journal 2020;133(9):1039-1043. [DOI: 10.1097/CM9.0000000000000774]

\section{Meng 2020 \{published data only\}}

Meng Z, Wang M, Song H, Guo S, Zhou Y, Li W, et al. Development and utilization of an intelligent application for aiding COVID-19 diagnosis. medRxiv [Preprint] 2020. [DOI: https://doi.org/10.1101/2020.03.18.20035816]

\section{Peng 2020 \{published data only\}}

Peng D, Zhang J, Xu Y, Liu Z, Wu P. Clinical analysis and early differential diagnosis of suspected pediatric patients with 2019 novel coronavirus infection. medRxiv [Preprint] 2020. [DOI: https://doi.org/10.1101/2020.04.07.20057315]

\section{Peng 2020a \{published data only\}}

Peng L, Liu KY, Xue F, Miao YF, Tu PA, Zhou C. Improved early recognition of coronavirus disease-2019 (COVID-19): singlecenter data from a Shanghai screening hospital. Archives of Iranian Medicine 2020;23(4):272-276. [DOI: 10.34172/ aim.2020.10]

\section{Shi 2020 \{published data only\}}

Shi Y, Tan M, Chen X, Liu Y, Huang J, Ou J, et al. Immunopathological characteristics of coronavirus disease 2019 cases in Guangzhou, China. medRxiv [Preprint] 2020. [DOI: https://doi.org/10.1101/2020.03.12.20034736] 
Song 2020 \{published data only\}

Song CY, Xu J, He JQ, Lu YQ. COVID-19 early warning score: a multi-parameter screening tool to identify highly suspected patients. medRxiv [Preprint] 2020. [DOI: https:// doi.org/10.1101/2020.03.05.20031906]

Spiezia 2020 \{published data only\}

Spiezia L, Boscolo A, Poletto F, Cerruti L, Tiberio I, Campello E, et al. COVID-19-Related severe hypercoagulability in patients admitted to intensive care unit for acute respiratory failure. Thrombosis and Haemostasis 2020;120(06):998-1000. [DOI: 10.1055/s-0040-1710018]

\section{Sun 2020 \{published data only\}}

Sun Y, Koh V, Marimuthu K, Ng OT, Young B, Vasoo S, et al. Epidemiological and clinical predictors of COVID-19. Clinical Infectious Diseases 2020;71(15):786-792. [DOI: https:// doi.org/10.1093/cid/ciaa322]

\section{Tang 2020 \{published data only\}}

Tang X, Du R, Wang R, Cao T, Guan L, Yang C, et al. Comparison of hospitalized patients with acute respiratory distress syndrome caused by COVID-19 and H1N1. Chest 2020;158(1):195-205. [DOI: https://doi.org/10.1016/ j.chest.2020.03.032]

\section{Wang 2020 \{published data only\}}

Wang Z, Weng J, Li Z, Hou R, Zhou L, Ye H, et al. Development and validation of a diagnostic nomogram to predict COVID-19 pneumonia. medRxiv [Preprint] 2020. [DOI: https:// doi.org/10.1101/2020.04.03.20052068]

\section{Wu 2020 \{published data only\}}

Wu J, Zhang P, Zhang L, Meng W, Li J, Tong C, et al. Rapid and accurate identification of COVID-19 infection through machine learning based on clinical available blood test results. medRxiv [Preprint] 2020. [DOI: https:// doi.org/10.1101/2020.04.02.20051136]

\section{Xu 2020 \{published data only\}}

Xu Y, Li Y, Zeng Q, Lu Z, Li Y, Wu W, et al. Clinical characteristics of SARS-CoV-2 pneumonia compared to controls in Chinese Han population. medRxiv [Preprint] 2020. [DOI: https:// doi.org/10.1101/2020.03.08.20031658]

\section{Yang 2020a \{published data only\}}

Yang Y, Shen C, Li J, Yuan J, Yang M, Wang F, et al. Exuberant elevation of IP-10, MCP-3 and IL-1ra during SARSCoV-2 infection is associated with disease severity and fatal outcome. medRxiv [Preprint] 2020. [DOI: https:// doi.org/10.1101/2020.03.02.20029975]

\section{Yin 2020 \{published data only\}}

Yin S, Huang M, Li D, Tang N. Difference of coagulation features between severe pneumonia induced by SARS-CoV2 and nonSARS-CoV2. Journal of Thrombosis and Thrombolysis 2020. [DOI: 10.1007/s11239-020-02105-8]

\section{Additional references}

\section{Covidence [Computer program]}

Veritas Health Innovation Covidence. Version accessed before 22 October 2020. Melbourne, Australia: Veritas Health Innovation. Available at covidence.org.

\section{Deeks 2020a}

Deeks JJ, Dinnes J, Takwoingi Y, Davenport C, Spijker R, TaylorPhillips $\mathrm{S}$, et al. Antibody tests for identification of current and past infection with SARS-CoV-2. Cochrane Database of Systematic Reviews 2020, Issue 6. Art. No: CD013652. [DOI: 10.1002/14651858.CD013652]

\section{Deeks 2020b}

Deeks JJ, Dinnes J, Takwoingi Y, Davenport C, Leeflang MMG, Spijker R, et al. Diagnosis of SARS-CoV-2 infection and COVID-19: accuracy of signs and symptoms; molecular, antigen, and antibody tests; and routine laboratory markers. Cochrane Database of Systematic Reviews 2020, Issue 4. Art. No: CD013596. [DOI: 10.1002/14651858.CD013596]

\section{Dinnes 2020}

Dinnes J, Deeks JJ, Adriano A, Berhane S, Davenport C, Dittrich $S$, et al. Rapid, point-of-care antigen and molecularbased tests for diagnosis of SARS-CoV-2 infection. Cochrane Database of Systematic Reviews 2020, Issue 8. Art. No: CD013705. [DOI: 10.1002/14651858.CD013705]

\section{Macaskill 2010}

Macaskill P, Gatsonis C, Deeks JJ, Harbord RM, Takwoingi Y Chapter 10: analysing and presenting results. In: Deeks JJ, Bossuyt PM, Gatsonis C editor(s). Cochrane Handbook for Systematic Reviews of Diagnostic Test Accuracy Version 1.0. The Cochrane Collaboration, 2010. Available from methods.cochrane.org/sdt/handbook-dta-reviews.

\section{McInnes 2020}

Salameh J-P, Leeflang MM, Hooft L, Islam N, McGrath TA, Pol CB, et al. Thoracic imaging tests for the diagnosis of COVID-19. Cochrane Database of Systematic Reviews 2020, Issue 9. Art. No: CD013639. [DOI: 10.1002/14651858.CD013639]

\section{Review Manager 2020 [Computer program]}

The Cochrane Collaboration Review Manager. Version 5.4. Copenhagen: The Cochrane Collaboration, 2020

\section{SAS 2015 [Computer program]}

SAS Institute SAS. Version 9.4. Cary, NC, USA: SAS Institute, 2015. Available at www.sas.com.

\section{Schünemann 2020a}

Schünemann HJ, Mustafa RA, Brozek J, Steingart KR, Leeflang M, Murad MH, et al. GRADE guidelines: 21 part 1. Study design, risk of bias, and indirectness in rating the certainty across a body of evidence for test accuracy. Journal of Clinical Epidemiology 2020;122:129-41. [PMID: 32060007]

\section{Schünemann 2020b}

Schünemann HJ, Mustafa RA, Brozek J, Steingart KR, Leeflang M, Murad MH, et al. GRADE guidelines: 21 part 2. 
Test accuracy: inconsistency, imprecision, publication bias, and other domains for rating the certainty of evidence and presenting it in evidence profiles and summary of findings tables. Journal of Clinical Epidemiology 2020;122:142-52. [PMID: 32058069]

\section{Struyf 2020}

Struyf T, Deeks JJ, Dinnes J, Takwoingi Y, Davenport C, Leeflang MM, et al. Signs and symptoms to determine if a patient presenting in primary care or hospital outpatient

\section{CHARACTERISTICS OF STUDIES}

Characteristics of included studies [ordered by study ID] settings has COVID-19 disease. Cochrane Database of Systematic Reviews 2020, Issue 7. Art. No: CD013665. [DOI: 10.1002/14651858.CD013665]

\section{Whiting 2011}

Whiting PF, Rutjes AW, Westwood ME, Mallett S, Deeks JJ, Reitsma JB, et al, QUADAS-2 Group. QUADAS-2: a revised tool for the quality assessment of diagnostic accuracy studies. Annals of Internal Medicine 2011;155(8):529-36.

Ai 2020b

\section{Study characteristics}

Patient Sampling

Study including patients suspected of having COVID-19, all suspected patients are classified between COVID-19 or not COVID-19 (single gate). Inclusion until February 9, 2020 and follow-up was until 20 March. Patients were hospitalized in a hospital in China (Xiangyang No.1 People's Hospital).

\section{Patient characteristics and setting}

Setting: hospital, not specified which department

Site: Xiangyang, Hubei province

Country: China

Symptoms and severity: not reported

Demographics: cases: $49 \%$ male, age: mean 50.3 years (SD 17.4). non-cases: $44 \%$ male,

age: mean 38.8 years (SD 20.1) - both children and adults Exposure history: cases: $75.9 \%$ had contact history. Non-cases: $41.5 \%$ had contact history

Time since onset of symptoms: not reported

Index tests

Routine laboratory tests (Table 2)

Blood routine examination results were before hospitalization, first enzyme level test results after hospitalization of these 2 groups; person doing the testing not stated. Hospital lab technicians processed samples. Thresholds for positivity or negativity were not reported but we assumed that the same thresholds were used as in Ai 2020b, which was a study on the same 102 participants with COVID-19.

\section{Target condition and reference standard(s)}

Reference standard: RT-PCR was used to confirm cases. For some cases, RT-PCR was repeated 5 times before a positive test was confirmed. Sample not reported.

Hence target condition was SARS-CoV-2 infection.
All participants received the RT-PCR to confirm diagnosis. It is not clear what the time interval between index and reference text is. Missing data for cases: lymphocytes +1 sample, PCT: 15 missing, ESR: 9 missing. Missing data for controls: ALT: 1 missing, AST: 4 more 
Ai 2020b (Continued)

Methodological quality

\begin{tabular}{llll}
\hline Item Authors' judgement & Risk of bias & $\begin{array}{l}\text { Applicability con- } \\
\text { cerns }\end{array}$ \\
\hline
\end{tabular}

DOMAIN 1: Patient Selection

Was a consecutive or random sample of patients en- Yes rolled?

\begin{tabular}{ll}
\hline Was a case-control design avoided? & Yes \\
\hline Did the study avoid inappropriate exclusions? & Yes
\end{tabular}

\section{Could the selection of patients have introduced Low risk}

bias?

Are there concerns that the included patients and

setting do not match the review question?

\section{DOMAIN 2: Index Test (All tests)}

Were the index test results interpreted without knowl- Unclear

edge of the results of the reference standard?

If a threshold was used, was it pre-specified? Unclear
Could the conduct or interpretation of the index test have introduced bias?

Are there concerns that the index test, its conduct, or interpretation differ from the review question?

\section{DOMAIN 3: Reference Standard}

Is the reference standards likely to correctly classify the No target condition?

Were the reference standard results interpreted with- Unclear out knowledge of the results of the index tests?
Could the reference standard, its conduct, or its in- terpretation have introduced bias?

\section{Are there concerns that the target condition as de- fined by the reference standard does not match the question?}

\section{DOMAIN 4: Flow and Timing}

Was there an appropriate interval between index test Unclear and reference standard?

Did all patients receive the same reference standard? Yes

Were all patients included in the analysis?

No 
Ai 2020b (Continued)

Could the patient flow have introduced bias?

High risk

Chen 2020c

\section{Study characteristics}

Patient Sampling

Patients suspected of having SARS-CoV-2 pneumonia and hospitalized at Chongqing Three Gorges Central Hospital from 26 to 31 January 2020 were included in our study.

Suspected $=(1)$ contact with Wuhan or surrounding areas of Wuhan or confirmed patient within 14 days from the onset of the disease; (2) with symptoms of fever or respiratory; (3) with imaging features of COVID-19

Patient characteristics and setting

Setting: hospital, not specified which department

Site: Chongqing Three Gorges Central Hospital

Country: China

Symptoms and severity: cases: $82.1 \%$ and $76.9 \%$, respectively of the participants had fever and cough. $10.3 \%$ had chest pains and $7.7 \%$ had diarrhoea. All the participants had clinical symptoms, such as sputum production, fatigue, shortness of breath, headache, arthralgia and vomiting. Controls: $53.8 \%$ and $46.2 \%$ had fever and cough respectively

Demographics: 78 COVID-19 patients and 26 controls. cases median age 45 (range 15-79) and controls was 61 years; $50 \%$ males in both cases and controls

Exposure history: $83.3 \%$ of COVID-19 patients admitted exposure to Wuhan (controls 26.9\%), among whom 48 participants resided in Wuhan, 3 participants had travelled to Wuhan, and 14 participants had contact with people in Wuhan before the onset of the disease within 14 days

Time since onset of symptoms: not reported

Routine laboratory tests (Table 2)

Data collection tables were based on electronic medical records. Person doing the testing, sample, timing of testing not stated

Target condition and reference standard(s)

2 consecutive positive nucleic acid test result of high-throughput sequencing or real-time RT-PCR assay; upper respiratory throat swab samples 2-6 times

Target condition is SARS-CoV-2 infection

Flow and timing

The time interval between index and reference test is not clear but likely short as all participants were already hospitalized. All participants received the same reference standard. No missed data noticed.

Comparative

Notes

Funding: Fundamental Research Funds for the Central Universities (Project No.2020CDJYGRH-YJ03 to Xianxiang Zhang); Natural Science Foundation of China (Grants No. 81972416, 81672554 and 81472417 to BH)

\section{Methodological quality}

Item Authors' judgement Risk of bias Applicability concerns

DOMAIN 1: Patient Selection 
Chen 2020c (Continued)

Was a consecutive or random sample of pa- Unclear tients enrolled?

\begin{tabular}{ll}
\hline Was a case-control design avoided? & Unclear \\
\hline Did the study avoid inappropriate exclusions? & Unclear
\end{tabular}

Could the selection of patients have intro- Unclear risk
duced bias?

duced bias?

\section{Are there concerns that the included pa- tients and setting do not match the review question?}

\section{DOMAIN 2: Index Test (All tests)}

\section{Were the index test results interpreted without Unclear} knowledge of the results of the reference standard?

\begin{tabular}{l} 
If a threshold was used, was it pre-specified? Unclear \\
\hline $\begin{array}{l}\text { Could the conduct or interpretation of the } \\
\text { index test have introduced bias? }\end{array}$ \\
$\begin{array}{l}\text { Are there concerns that the index test, its } \\
\text { conduct, or interpretation differ from the re- } \\
\text { view question? }\end{array}$
\end{tabular}

\section{DOMAIN 3: Reference Standard} Is the reference standards likely to correctly No
classify the target condition?

Were the reference standard results interpret- Unclear ed without knowledge of the results of the index tests?

\section{Could the reference standard, its conduct, or its interpretation have introduced bias?}

High risk

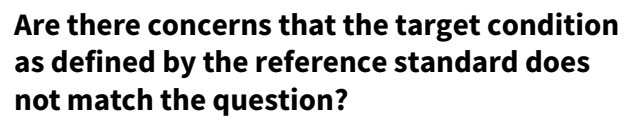




\section{Study characteristics}

Patient Sampling

Patients suspected of having SARS-CoV-2 pneumonia and hospitalized at Chongqing Three Gorges Central Hospital from 26 to 31 January 2020 were included in our study.

Suspected $=(1)$ contact with Wuhan or surrounding areas of Wuhan or confirmed patient within 14 days from the onset of the disease; (2) with symptoms of fever or respiratory; (3) with imaging features of COVID-19

Patient characteristics and setting

Setting: hospital, not specified which department

Site: Chongqing Three Gorges Central Hospital

Country: China

Symptoms and severity: cases: $82.1 \%$ and $76.9 \%$, respectively of the participants had fever and cough. $10.3 \%$ had chest pains and $7.7 \%$ had diarrhoea. All the participants had clinical symptoms, such as sputum production, fatigue, shortness of breath, headache, arthralgia and vomiting. Controls: $53.8 \%$ and $46.2 \%$ had fever and cough respectively Demographics: 78 COVID-19 patients and 26 controls. cases median age 45 (range 15-79) and controls was 61 years; $50 \%$ males in both cases and controls

Exposure history: $83.3 \%$ of COVID-19 patients admitted exposure to Wuhan (controls 26.9\%), among whom 48 participants resided in Wuhan, 3 participants had travelled to Wuhan, and 14 participants had contact with people in Wuhan before the onset of the disease within 14 days

Time since onset of symptoms: not reported

Index tests Routine laboratory tests (Table 2)

Data collection tables were based on electronic medical records. Person doing the testing, sample, timing of testing not stated

Target condition and reference standard(s) 2 consecutive positive nucleic acid test result of high-throughput sequencing or real-time RT-PCR assay; upper respiratory throat swab samples 2-6 times

Target condition is SARS-CoV-2 infection

Flow and timing

The time interval between index and reference test is not clear but likely short as all participants were already hospitalized. All participants received the same reference standard. No missed data noticed.

Comparative

Notes

Funding: Fundamental Research Funds for the Central Universities (Project No.2020CDJYGRH-YJ03 to Xianxiang Zhang); Natural Science Foundation of China (Grants No. 81972416,81672554 and 81472417 to $\mathrm{BH}$ )

\section{Methodological quality}

\begin{tabular}{llll}
\hline Item & Authors' judgement & Risk of bias & Applicability concerns \\
\hline
\end{tabular}

DOMAIN 1: Patient Selection

Was a consecutive or random sample of pa- Unclear
tients enrolled?

tients enrolled? 
Chen 2020c (Continued)

Did the study avoid inappropriate exclusions? Unclear

Could the selection of patients have intro- Unclear risk
duced bias?

duced bias?

Are there concerns that the included pa-

Low concern

tients and setting do not match the review

question?

\section{DOMAIN 2: Index Test (All tests)}

Were the index test results interpreted without Unclear

knowledge of the results of the reference standard?

If a threshold was used, was it pre-specified? Unclear

Could the conduct or interpretation of the Unclear risk
index test have introduced bias?

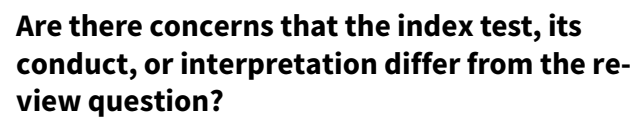

\section{DOMAIN 3: Reference Standard}

Is the reference standards likely to correctly No

classify the target condition?

\section{Were the reference standard results interpret- Unclear}

ed without knowledge of the results of the in-

dex tests?

Could the reference standard, its conduct, or $\quad$ High risk
its interpretation have introduced bias?

Could the reference standard, its conduct, or High risk
its interpretation have introduced bias?

Are there concerns that the target condition
as defined by the reference standard does
not match the question?

not match the question?

\section{DOMAIN 4: Flow and Timing}

Was there an appropriate interval between in- Unclear dex test and reference standard?

Did all patients receive the same reference Yes

standard?

Were all patients included in the analysis?

Yes

Could the patient flow have introduced bias?

Unclear risk

\section{Study characteristics}


Feng 2020 (Continued)

Patient Sampling
Study including adult patients with suspected infection, all suspected patients are classified between COVID-19 or not COVID-19 (single gate). Between 14 January and 9 February

All patients admitted to the fever clinic of emergency department of the First Medical Center, Chinese People's Liberation Army General Hospital (PLAGH) in Beijing with the epidemiological history of exposure to COVID-19 according to WHO interim guidance were enrolled in this study.

\section{Patient characteristics and setting}

Setting: fever clinic of emergency department

Site: First Medical Center, Chinese People's Liberation Army General Hospital (PLAGH) in Beijing

Country: China

Symptoms and severity: all 7 cases had moderate disease as defined by the 6 th-Guidelines-CNHHC

Demographics: 7 cases and 19 controls. Median age: 39 years in cases and 40 years for controls. Cases were $71.4 \%$ male and controls were $63.2 \%$ male (adults only)

Exposure history: history of sojourn or residence: $57.1 \%$ for cases and $21.1 \%$ for controls. History of contact with confirmed patient: cases: $28.6 \%$ and controls $5.3 \%$. History of contact with person who had fever or respiratory symptoms: cases $14.3 \%$ and controls $57.9 \%$

Time since onset of symptoms: not reported. Days from illness onset to first admission: median 5 days for cases and 1 day for controls
Routine laboratory tests (Table 2)

Lymphocyte count (LYMPH\#), CRP and IL-6 were evaluated on admission. Lymphopenia $\left(<1.0 \times 10^{9} / \mathrm{L}\right)$ was 1 of the 3 diagnostic criteria for S-COVID-19-P according to the 6th-Guidelines-CNHHC. Elevated CRP $(>0.8 \mathrm{mg} / \mathrm{L})$ and elevated IL-6 $(>5.9 \mathrm{pg} / \mathrm{mL})$ were both important infection-related biomarkers

\section{Target condition and reference standard(s) Target condition: S-COVID-19-P}

COVID-19 infection was confirmed by real-time RT-PCR using the same protocol described previously (Huang C, Wang Y, Li X, et al. Clinical features of patients infected with 2019 novel coronavirus in Wuhan, China. Lancet 2020; 395(10223): 497-506.). RTPCR detection reagents were provided by the four institutions. Not clear how other criteria were included in the diagnosis.

Flow and timing

Nothing reported about flow and timing.

Comparative

Notes

Funding: the present study was supported by grants from the PLA Science and Technology Project (14CXZ005, AWS15J004, 16BJZ19), National Key R\&D Program of China 2019YFF0302300), Construction Project of Key Disciplines in the 13th Five-Year Plan of the PLA (Traumatic Surgery in the Battlefield, 2019-126, 2019-513), Beijing Science and Technology New Star Project (XX2018019/Z181100006218028), the PLA General Hospital Science and technology Project (2019XXJSYX20, 2018XXFC-20, ZH19016).

\section{Methodological quality}

\begin{tabular}{llll}
\hline Item & Authors' judgement & Risk of bias
\end{tabular}

DOMAIN 1: Patient Selection

Was a consecutive or random sample of Yes patients enrolled? 
Feng 2020 (Continued)

Was a case-control design avoided? Yes

Did the study avoid inappropriate exclu- Yes sions?

Could the selection of patients have in- Low risk
troduced bias?

Are there concerns that the included pa-

Low concern

tients and setting do not match the re-

view question?

\section{DOMAIN 2: Index Test (All tests)}

Were the index test results interpreted

Yes

without knowledge of the results of the ref-

erence standard?

If a threshold was used, was it pre-speci- Yes

fied?

Could the conduct or interpretation of

Low risk

the index test have introduced bias?

\section{Are there concerns that the index test, its conduct, or interpretation differ from \\ the review question?}

\section{DOMAIN 3: Reference Standard}

\section{Is the reference standards likely to correct- Unclear}

ly classify the target condition?

\section{Were the reference standard results inter- Unclear} preted without knowledge of the results of the index tests?

\section{Could the reference standard, its con- duct, or its interpretation have intro- duced bias?}

\section{Are there concerns that the target con- dition as defined by the reference stan- dard does not match the question?}

\section{DOMAIN 4: Flow and Timing}

Was there an appropriate interval between Yes index test and reference standard?

Did all patients receive the same reference Yes standard?

Were all patients included in the analysis? Yes

Could the patient flow have introduced Low risk
bias?

bias? 


\section{Study characteristics}

Patient Sampling

Study including adult patients with suspected infection, all suspected patients are classified between COVID-19 or not COVID-19 (single gate). Between 14 January and 9 February

All patients admitted to the fever clinic of emergency department of the First Medical Center, Chinese People's Liberation Army General Hospital (PLAGH) in Beijing with the epidemiological history of exposure to COVID-19 according to WHO interim guidance were enrolled in this study.

Patient characteristics and setting

Setting: fever clinic of emergency department

Site: First Medical Center, Chinese People's Liberation Army General Hospital (PLAGH)

in Beijing

Country: China

Symptoms and severity: all 7 cases had moderate disease as defined by the 6th-Guidelines-CNHHC

Demographics: 7 cases and 19 controls. Median age: 39 years in cases and 40 years for controls. Cases were $71.4 \%$ male and controls were $63.2 \%$ male (adults only)

Exposure history: history of sojourn or residence: $57.1 \%$ for cases and $21.1 \%$ for controls. History of contact with confirmed patient: cases: $28.6 \%$ and controls $5.3 \%$. History of contact with person who had fever or respiratory symptoms: cases $14.3 \%$ and controls $57.9 \%$

Time since onset of symptoms: not reported. Days from illness onset to first admission: median 5 days for cases and 1 day for controls

Lymphocyte count (LYMPH\#), CRP and IL-6 were evaluated on admission. Lymphopenia $(<1.0 \times 109 / \mathrm{L})$ was 1 of the 3 diagnostic criteria for S-COVID-19-P according to the 6th-Guidelines-CNHHC. Elevated CRP $(>0.8 \mathrm{mg} / \mathrm{L})$ and elevated IL-6 $(>5.9 \mathrm{pg} / \mathrm{mL})$ were both important infection-related biomarkers

Target condition and reference standard(s) Target condition: S-COVID-19-P

COVID-19 infection was confirmed by real-time RT-PCR using the same protocol described previously (Huang C, Wang Y, Li X, et al. Clinical features of patients infected with 2019 novel coronavirus in Wuhan, China. Lancet 2020; 395(10223): 497-506.). RTPCR detection reagents were provided by the four institutions. Not clear how other criteria were included in the diagnosis.

Flow and timing

Nothing reported about flow and timing.

Comparative

Notes

Funding: the present study was supported by grants from the PLA Science and Technology Project (14CXZ005, AWS15J004, 16BJZ19), National Key R\&D Program of China 2019YFF0302300), Construction Project of Key Disciplines in the 13th Five-Year Plan of the PLA (Traumatic Surgery in the Battlefield, 2019-126, 2019-513), Beijing Science and Technology New Star Project (XX2018019/Z181100006218028), the PLA General Hospital Science and technology Project (2019XXJSYX20, 2018XXFC-20, ZH19016).

\section{Methodological quality}

\begin{tabular}{llll}
\hline Item & Authors' judgement & Risk of bias & Applicability concerns \\
\hline
\end{tabular}


Feng 2020 (Continued)

\section{DOMAIN 1: Patient Selection}

Was a consecutive or random sample of Yes
patients enrolled?

\begin{tabular}{ll}
\hline Was a case-control design avoided? & Yes \\
\hline $\begin{array}{l}\text { Did the study avoid inappropriate exclu- } \\
\text { sions? }\end{array}$ & Yes
\end{tabular}

\section{Could the selection of patients have in- Low risk troduced bias?}

\section{Are there concerns that the included pa- tients and setting do not match the re- \\ view question?}

\section{DOMAIN 2: Index Test (All tests)}

Were the index test results interpreted Yes

without knowledge of the results of the ref-

erence standard?

If a threshold was used, was it pre-speci- Yes fied?

\section{Could the conduct or interpretation of} the index test have introduced bias?

\section{Are there concerns that the index test, its conduct, or interpretation differ from the review question?}

\section{DOMAIN 3: Reference Standard}

Is the reference standards likely to correct- Unclear ly classify the target condition?

Were the reference standard results inter- Unclear preted without knowledge of the results of the index tests?

Could the reference standard, its con-
duct, or its interpretation have intro-
duced bias?

Are there concerns that the target condition as defined by the reference standard does not match the question?

\section{DOMAIN 4: Flow and Timing}

Was there an appropriate interval between Yes index test and reference standard?

Did all patients receive the same reference Yes standard? 
Feng 2020 (Continued)

Were all patients included in the analysis? Yes

\section{Could the patient flow have introduced}

bias?

Low risk

Ferrari 2020

\section{Study characteristics}

\section{Patient Sampling}

Study including suspected patients, all suspected patients are classified between COVID-19 or not COVID-19 (single-gate, case-control design). Between 20 February and 20 March 2020

The participants were randomly chosen (alphabetical order) to have a similar number of individuals in the positive (105) and negative (102) rRT-PCR test groups

\section{Patient characteristics and setting}

Setting: fever clinic of emergency department

Site: San Raffaele Hospital (Milan, Italy) emergency room

Country: Italy

Symptoms and severity: currently Italy has strict directives suggesting an rRT-PCR test only if patients show $\geq 3$ ARS symptoms, review authors assumed that most, if not all, of the individuals enrolled in this study went to the hospital emergency room with fever, cough and fatigue.

Demographics: median age for cases is 61.8 and for controls is 59.2 cases: $70.5 \%$ male and controls $52 \%$ male (adults only)

Exposure history: not stated

Time since onset of symptoms: not reported
Routine laboratory tests (Table 2)

Blood samples were collected on the same day of the rRT-PCR test. CRP, AST, ALT, GGT, ALP and LDH were measured on a Roche Cobas 8000 device (Roche Diagnostic, Basel, Switzerland) using either a spectrophotometric assay (AST, ALT and $\mathrm{LDH}$ ), a colorimetric assay (ALP and GGT) or an immunoturbidimetric assay (CRP). WBC, platelets and the leukocyte formula were measured on Sysmex XE 2100 (Sysmex, Japan).

\footnotetext{
Target condition and reference standard(s)
}

\section{Target condition: SARS-CoV-2 infection}

Reference standard: rRT-PCR was performed on a Roche Cobas Z480 thermocycler (Roche Diagnostic, Basel, Switzerland) using the Roche-provided Tib-Molbiol's 2019-nCoV Real-Time Reverse Transcription PCR Kit. RNA purification was performed using the Roche Magna pure system.

Number of samples tested per participant not reported; blinding not reported; no other criteria used.

Flow and timing Blood samples were collected on the same day of the rRT-PCR test; none missing

Comparative

Notes
We could not extract $2 \times 2$ table because study only reported means and SDs. Study authors contacted; they sent data for 2 tests

\section{Methodological quality}


Ferrari 2020 (Continued)

Item Authors' judgement Risk of bias $\quad$ Applicability concerns

\section{DOMAIN 1: Patient Selection}

Was a consecutive or random sample of patients Yes enrolled?

\begin{tabular}{ll}
\hline Was a case-control design avoided? & Yes \\
\hline Did the study avoid inappropriate exclusions? & Yes \\
\hline
\end{tabular}

Could the selection of patients have intro- Low risk
duced bias?

\section{Are there concerns that the included patients} and setting do not match the review ques-

tion?

\section{DOMAIN 2: Index Test (All tests)}

Were the index test results interpreted without knowledge of the results of the reference standard?

If a threshold was used, was it pre-specified? Unclear

Could the conduct or interpretation of the in- Unclear risk
dex test have introduced bias?

Are there concerns that the index test, its conduct, or interpretation differ from the review question?

\section{DOMAIN 3: Reference Standard} Is the reference standards likely to correctly No
classify the target condition?

Were the reference standard results interpret- Unclear ed without knowledge of the results of the index tests?
Could the reference standard, its conduct, or its interpretation have introduced bias?

\section{Are there concerns that the target condition as defined by the reference standard does not match the question?}

\section{DOMAIN 4: Flow and Timing}

Was there an appropriate interval between index Yes test and reference standard?

Did all patients receive the same reference stan- Yes dard? 
Ferrari 2020 (Continued)

Were all patients included in the analysis?

Yes

Could the patient flow have introduced bias?

Low risk

\section{Ferrari 2020}

\section{Study characteristics}

Patient Sampling

Patient characteristics and setting
Study including suspected patients, all suspected patients are classified between COVID-19 or not COVID-19 (single-gate, case-control design). Between 20 February and 20 March 2020

The participants were randomly chosen (alphabetical order) to have a similar number of individuals in the positive (105) and negative (102) rRT-PCR test groups

Setting: fever clinic of emergency department

Site: San Raffaele Hospital (Milan, Italy) emergency room

Country: Italy

Symptoms and severity: currently Italy has strict directives suggesting an rRT-PCR test only if patients show $\geq 3$ ARS symptoms, review authors assumed that most, if not all, of the individuals enrolled in this study went to the hospital emergency room with fever, cough and fatigue.

Demographics: median age for cases is 61.8 and for controls is 59.2 cases: $70.5 \%$ male and controls $52 \%$ male (adults only)

Exposure history: not stated

Time since onset of symptoms: not reported

\section{Routine laboratory tests (Table 2)}

Blood samples were collected on the same day of the rRT-PCR test. CRP, AST, ALT, GGT, ALP and LDH were measured on a Roche Cobas 8000 device (Roche Diagnostic, Basel, Switzerland) using either a spectrophotometric assay (AST, ALT and LDH), a colorimetric assay (ALP and GGT) or an immunoturbidimetric assay (CRP). WBC, platelets and the leukocyte formula were measured on Sysmex XE 2100 (Sysmex, Japan).

\footnotetext{
Target condition and reference standard(s)
}

\section{Target condition: SARS-CoV-2 infection}

Reference standard: rRT-PCR was performed on a Roche Cobas Z480 thermocycler (Roche Diagnostic, Basel, Switzerland) using the Roche-provided Tib-Molbiol's 2019-nCoV Real-Time Reverse Transcription PCR Kit. RNA purification was performed using the Roche Magna pure system.

Number of samples tested per participant not reported; blinding not reported; no other criteria used.

Blood samples were collected on the same day of the rRT-PCR test; none missing
We could not extract $2 \times 2$ table because study only reported means and SDs. Study authors contacted; they sent data for 2 tests

\section{Methodological quality}

\begin{tabular}{llll} 
Item & Authors' judgement & Risk of bias & Applicability concerns \\
\hline
\end{tabular}


Ferrari 2020 (Continued)

\section{DOMAIN 1: Patient Selection}

Was a consecutive or random sample of patients Yes enrolled?

\begin{tabular}{ll}
\hline Was a case-control design avoided? & Yes \\
\hline Did the study avoid inappropriate exclusions? & Yes \\
\hline
\end{tabular}

Could the selection of patients have intro- Low risk
duced bias?

Are there concerns that the included patients
and setting do not match the review ques-
tion?

\begin{tabular}{ll}
\hline Could the conduct or interpretation of the in- & Unclear risk \\
dex test have introduced bias?
\end{tabular}

\section{DOMAIN 3: Reference Standard}

Is the reference standards likely to correctly No classify the target condition?

Were the reference standard results interpret- Unclear ed without knowledge of the results of the index tests?

Could the reference standard, its conduct, or its interpretation have introduced bias?

\section{Are there concerns that the target condition as defined by the reference standard does not match the question?}

\section{DOMAIN 4: Flow and Timing}

Was there an appropriate interval between index Yes test and reference standard?

Did all patients receive the same reference stan- Yes dard? 
Ferrari 2020 (Continued)

\section{Study characteristics}

Patient Sampling
Patients admitted to China Medical University Hospital meeting the screening criteria of COVID-19 reported by Taiwan CDC (travel history to China and presented fever or any respiratory symptoms within 14 days). All eligible patients were included.

Setting: hospital, emergency room

Country: Taiwan

Symptoms and severity: most common symptoms were fever, nonproductive cough, rhinorrhoea, sore throat, productive cough and dyspnea Demographics: mean age 34 (range 3-68), female 60\%

Exposure history: travel to China, contact with people travelling to China, or contact with COVID-19 patients

Time since onset of symptoms: not reported
Index tests (threshold):

- WBC count increased $\left(11.2 \times 10^{9} / \mathrm{L}\right)$

- WBC count decreased (3.6 $\left.\times 10^{9} / \mathrm{L}\right)$

- Lymphocyte count decreased (1.0 x 109/L)

- CRP increased (10 mg/L)

For all tests

- Sample: blood product, whole blood (not reported, but otherwise WBC impossible)

- Test interpreter: not reported

- Timing of testing: not reported

\section{Target condition and reference standard(s)}

RT-PCR (conducted multiple times in each participant; at least upon admission and $24 \mathrm{~h}$ after admission, and for some participants even every few days). Target condition was SARS-CoV-2 infection.

Sample: naso-oropharyngeal specimen, sputum Threshold: not reported

Flow and timing

Time interval between index test and reference standard: not clearly reported

Verification: all participants received the same reference standard Missing data: no missing data or uninterpretable results

\section{Comparative}

Notes

Funding: this study was supported by a grant, CMUH DMR-108-189, from

China Medical University Hospital, Taichung, Taiwan.

\section{Methodological quality}

\section{Item}

Authors' judgement

Risk of bias

\section{Applicability con-} cerns 
Hsih 2020 (Continued)

\title{
DOMAIN 1: Patient Selection
}

Was a consecutive or random sample of patients en- $\quad$ Yes
rolled?
rolled?

\begin{tabular}{lll}
\hline Was a case-control design avoided? & Yes \\
\hline Did the study avoid inappropriate exclusions? & Yes & \\
\hline Could the selection of patients have introduced bias? & Low risk \\
\hline $\begin{array}{l}\text { Are there concerns that the included patients and set- } \\
\text { ting do not match the review question? }\end{array}$ & Unclear \\
\hline
\end{tabular}

\section{DOMAIN 2: Index Test (All tests)}

Were the index test results interpreted without knowl- Unclear edge of the results of the reference standard?

If a threshold was used, was it pre-specified? Unclear

Could the conduct or interpretation of the index test Unclear risk
have introduced bias?

\author{
have introduced bias?
}

Are there concerns that the index test, its conduct, or interpretation differ from the review question?

\section{DOMAIN 3: Reference Standard}

Is the reference standards likely to correctly classify the No target condition?

Were the reference standard results interpreted without Unclear knowledge of the results of the index tests?
Could the reference standard, its conduct, or its inter- pretation have introduced bias?

\section{DOMAIN 4: Flow and Timing}

Was there an appropriate interval between index test Unclear and reference standard?

\begin{tabular}{ll}
\hline Did all patients receive the same reference standard? & Yes \\
\hline Were all patients included in the analysis? & Yes
\end{tabular}

Could the patient flow have introduced bias? Unclear risk




\section{Study characteristics}

\section{Patient Sampling}

Children with confirmed 2019-nCoV pneumonia (cases) admitted between 24 January and 22 February 2020 and children with RSV pneumonia (controls) admitted between 10 December 2019 and 22 February 2020 in Wuhan Children's hospital and patients who underwent the detection of peripheral blood lymphocyte subsets were included in the study. Previously healthy children were included in the study, and children receiving chemotherapy, treatment of glucocorticoids or immunosuppressant before the diagnosis of the pneumonia were not included in the study as their immune response to viral infections might be different.
Setting: Wuhan Children's hospital

Site: Wuhan

Country: China

Symptoms and severity: of all children, 3 participants developed severe pneumonia, $1(2.5 \%)$ in cases and $2(12.5 \%)$ in control

Demographics: cases $57 \%$ male; controls $62.5 \%$ male

Age: cases: mean age 5.09 years and controls 1.36 years

Exposure history: not stated

Time since onset of symptoms: not stated

Any other info:

Whole blood

Demographic data, clinical manifestations, laboratory findings (including CRP, PCT, Scr, ALT, lymphocyte subsets, cytokines (IL-2, IL-4, IL-6, IL-10,TNF- $\alpha$, IFN- $\gamma$ ) ) and treatments were recorded from the medical records

Cytokines may not be standard in all places, hence unclear concerns regarding applicability.

Target condition and reference standard(s)
Real-time RT-PCR; not reported how often sampled; not reported about blinding.

Also, 2019-nCoV infection was confirmed with RT-PCR, but unclear how 2019-nCoV was defined in the first place, before confirming

Cases and controls were selected based on detection of peripheral blood

lymphocyte subsets. Time interval unclear, but likely before RT-PCR test

Flow and timing

\section{Comparative}

Notes

\section{Methodological quality}

\begin{tabular}{llll}
\hline Item Authors' judgement & Risk of bias & $\begin{array}{l}\text { Applicability con- } \\
\text { cerns }\end{array}$
\end{tabular}

\section{DOMAIN 1: Patient Selection}

Was a consecutive or random sample of patients en- Unclear
rolled? 
Li 2020d (Continued)

Did the study avoid inappropriate exclusions?

Unclear

\begin{tabular}{|c|c|}
\hline $\begin{array}{l}\text { Could the selection of patients have introduced } \\
\text { bias? }\end{array}$ & High risk \\
\hline
\end{tabular}

bias?

Are there concerns that the included patients and

setting do not match the review question?

\section{DOMAIN 2: Index Test (All tests)}

Were the index test results interpreted without knowl- No

edge of the results of the reference standard?

If a threshold was used, was it pre-specified? Unclear

$\begin{aligned} & \text { Could the conduct or interpretation of the index } \\ & \text { test have introduced bias? }\end{aligned}$

Are there concerns that the index test, its conduct, or interpretation differ from the review question?

\section{DOMAIN 3: Reference Standard}

Is the reference standards likely to correctly classify No the target condition?

Were the reference standard results interpreted with- Unclear out knowledge of the results of the index tests?

Could the reference standard, its conduct, or its in-
terpretation have introduced bias?

Are there concerns that the target condition as de-
fined by the reference standard does not match the
question?

\section{DOMAIN 4: Flow and Timing}

Was there an appropriate interval between index test Unclear and reference standard?

\begin{tabular}{lll}
\hline Did all patients receive the same reference standard? & Unclear \\
\hline Were all patients included in the analysis? & Yes & Unclear risk \\
\hline Could the patient flow have introduced bias? & & \\
\hline
\end{tabular}

\section{Li 2020e}

\section{Study characteristics}

Patient Sampling Pregnant women who were admitted into the Hubei Provincial Maternal and Child Health Center, during 24 January-29 February 2020. The study also included suspected patients with typical chest CT imaging but negative in RT-PCR tests. Eleven pregnant women who were tested positive for SARS-CoV-2 were classified as labo- 
ratory-confirmed case group, and eighteen with typical chest $\mathrm{CT}$ imaging but tested negative in RT-PCR tests as suspected case group.

The control group of pregnant women without pneumonia during hospital stay were randomly selected from the medical records by an investigator (MP), who was not involved in statistical analysis. Only those aged 25-35 years were selected to match the age range of cases. 121 women admitted during 24 January-11 February 2019 (control 2019 group)

Patient characteristics and setting

Pregnant women (and therefore high concern regarding applicability)

Setting: admission to hospital

Site: Hubei Provincial Maternal and Child Health Center

Country: China

Symptoms and severity: 4 of the cases were admitted with fever for investigation and 8 developed fever after childbirth. None presented other respiratory symptoms on admission nor during hospital stay. 2 of the patients with suspected COVID-19 pneumonia reported cough, sore throat, dyspnea, diarrhea and vomiting. Demographics: pregnant women

Age: confirmed cases: 30.9 years, suspected cases 29.8 years. Control 1:30.1 years and control 2: 29.3 years

Exposure history: none of confirmed COVID-19 patients reported an exposure history. Retrospective analysis of medical records of pregnant women with COVID-19 pneumonia and pregnant women without COVID-19 pneumonia.

Time since onset of symptoms: not reported were collected from medical records and reviewed independently by 2 investigators Index tests were: WBC, lymphocytes, neutrophils, CRP, eosinophils, ALT, AST

\footnotetext{
Target condition and reference standard(s)
}

\section{Cases: RT-PCR and chest CT}

Controls: 121 women admitted during 24 January-11 February 2019 (control 2019 group)

Target condition: COVID-19

Flow and timing Blood test results were also retrieved from medical records. 2 case groups underwent blood tests every three days but 2 control groups only taken once

\section{Comparative}

Notes

\section{Methodological quality}

\begin{tabular}{|c|c|c|c|}
\hline Item & Authors' judgement & Risk of bias & Applicability concerns \\
\hline \multicolumn{4}{|l|}{ DOMAIN 1: Patient Selection } \\
\hline $\begin{array}{l}\text { Was a consecutive or random sample of pa- } \\
\text { tients enrolled? }\end{array}$ & Unclear & & \\
\hline Was a case-control design avoided? & No & & \\
\hline Did the study avoid inappropriate exclusions? & No & & \\
\hline
\end{tabular}


Li 2020e (Continued)

Could the selection of patients have intro-

High risk duced bias?

Are there concerns that the included pa-

question?

\section{DOMAIN 2: Index Test (All tests)}

Were the index test results interpreted with-

standard?

If a threshold was used, was it pre-specified? Unclear

Could the conduct or interpretation of the Unclear risk
index test have introduced bias?

index test have introduced bias?

Are there concerns that the index test, its conduct, or interpretation differ from the review question?

\section{DOMAIN 3: Reference Standard}

Is the reference standards likely to correctly Yes

classify the target condition?

\section{Were the reference standard results interpret- Unclear} ed without knowledge of the results of the index tests?

\section{Could the reference standard, its conduct, or its interpretation have introduced bias?}

\section{Are there concerns that the target condi- tion as defined by the reference standard does not match the question?}

\section{DOMAIN 4: Flow and Timing}

Was there an appropriate interval between in- Yes dex test and reference standard?

Did all patients receive the same reference No standard?

Were all patients included in the analysis? Yes

\begin{tabular}{|c|}
\hline $\begin{array}{l}\text { Could the patient flow have introduced } \\
\text { bias? }\end{array}$ \\
\hline
\end{tabular}

\section{Study characteristics}


Hsih 2020 (Continued)

Patient Sampling
Patients admitted to China Medical University Hospital meeting the screening criteria of COVID-19 reported by Taiwan CDC (travel history to China and presented fever or any respiratory symptoms within 14 days). All eligible patients were included.

\section{Patient characteristics and setting}

Setting: hospital, emergency room

Country: Taiwan

Symptoms and severity: most common symptoms were fever, nonproductive cough, rhinorrhoea, sore throat, productive cough and dyspnea Demographics: mean age 34 (range 3-68), female 60\%

Exposure history: travel to China, contact with people travelling to China, or contact with COVID-19 patients

Time since onset of symptoms: not reported
Index tests (threshold):

- WBC count increased $\left(11.2 \times 10^{9} / \mathrm{L}\right)$

- WBC count decreased (3.6 $\left.\times 10^{9} / \mathrm{L}\right)$

- Lymphocyte count decreased $\left(1.0 \times 10^{9} / \mathrm{L}\right)$

- CRP increased (10 mg/L)

For all tests

- Sample: blood product, whole blood (not reported, but otherwise WBC impossible)

- Test interpreter: not reported

- Timing of testing: not reported
Target condition and reference standard(s)
RT-PCR (conducted multiple times in each participant; at least upon admission and $24 \mathrm{~h}$ after admission, and for some participants even every few days). Target condition was SARS-CoV-2 infection.

Sample: naso-oropharyngeal specimen, sputum Threshold: not reported

Time interval between index test and reference standard: not clearly reported

Verification: all participants received the same reference standard Missing data: no missing data or uninterpretable results

\section{Comparative}

Notes

Funding: this study was supported by a grant, CMUH DMR-108-189, from China Medical University Hospital, Taichung, Taiwan.

\section{Methodological quality}

\begin{tabular}{llll}
\hline Item Authors' judgement & Risk of bias & $\begin{array}{l}\text { Applicability con- } \\
\text { cerns }\end{array}$
\end{tabular}

\section{DOMAIN 1: Patient Selection}

Was a consecutive or random sample of patients enrolled?
Yes

Yes

Yes

Did the study avoid inappropriate exclusions?

Routine laboratory testing to determine if a patient has COVID-19 (Review) 
Hsih 2020 (Continued)

Could the selection of patients have introduced bias?

Low risk

Are there concerns that the included patients and set-

Unclear ting do not match the review question?

\section{DOMAIN 2: Index Test (All tests)}

Were the index test results interpreted without knowl- Unclear

edge of the results of the reference standard?

If a threshold was used, was it pre-specified? Unclear

Could the conduct or interpretation of the index test
have introduced bias?

Could the conduct or interpretation of the index test
have introduced bias?

Are there concerns that the index test, its conduct, or

Unclear interpretation differ from the review question?

\section{DOMAIN 3: Reference Standard}

Is the reference standards likely to correctly classify the No target condition?

Were the reference standard results interpreted without Unclear knowledge of the results of the index tests?

Could the reference standard, its conduct, or its inter-
pretation have introduced bias?

Are there concerns that the target condition as de-

fined by the reference standard does not match the question?

\section{DOMAIN 4: Flow and Timing}

Was there an appropriate interval between index test Unclear
and reference standard?
and reference standard?

\begin{tabular}{ll}
\hline Did all patients receive the same reference standard? & Yes \\
\hline Were all patients included in the analysis? & Yes \\
\hline Could the patient flow have introduced bias? & \\
\hline
\end{tabular}

\section{Li 2020d}

\section{Study characteristics}

Patient Sampling
Children with confirmed 2019-nCoV pneumonia (cases) admitted between 24 January and 22 February 2020 and children with RSV pneumonia (controls) admitted between 10 December 2019 and 22 February 2020 in Wuhan Children's hospital and patients who underwent the detection of peripheral blood lymphocyte subsets were included in the study. Previously healthy children were included in the study, and children receiving chemotherapy, treatment of glucocorticoids or immunosuppressant before the diagnosis of 
the pneumonia were not included in the study as their immune response to viral infections might be different.

Patient characteristics and setting
Setting: Wuhan Children's hospital

Site: Wuhan

Country: China

Symptoms and severity: of all children, 3 participants developed severe pneumonia, $1(2.5 \%)$ in cases and $2(12.5 \%)$ in control

Demographics: cases $57 \%$ male; controls $62.5 \%$ male

Age: cases: mean age 5.09 years and controls 1.36 years

Exposure history: not stated

Time since onset of symptoms: not stated

Any other info:
Index tests
Whole blood

Demographic data, clinical manifestations, laboratory findings (including CRP, PCT, Scr, ALT, lymphocyte subsets, cytokines (IL-2, IL-4, IL-6, IL-10,TNF- $\alpha$, IFN- $\gamma$ ) ) and treatments were recorded from the medical records

Cytokines may not be standard in all places, hence unclear concerns regarding applicability.
Real-time RT-PCR; not reported how often sampled; not reported about blinding.

Also, 2019-nCoV infection was confirmed with RT-PCR, but unclear how 2019-nCoV was defined in the first place, before confirming

Cases and controls were selected based on detection of peripheral blood lymphocyte subsets. Time interval unclear, but likely before RT-PCR test

Flow and timing

\section{Comparative}

Notes

\section{Methodological quality}

\begin{tabular}{llll}
\hline Item Authors' judgement & Risk of bias & $\begin{array}{l}\text { Applicability con- } \\
\text { cerns }\end{array}$ \\
\hline
\end{tabular}

DOMAIN 1: Patient Selection Was a consecutive or random sample of patients en- Unclear
rolled?

\begin{tabular}{ll}
\hline Was a case-control design avoided? & No \\
\hline Did the study avoid inappropriate exclusions? & Unclear
\end{tabular}

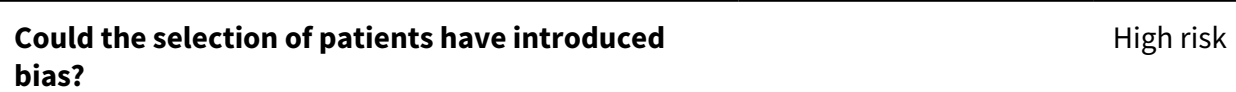

bias? 
Setting: admission to hospital

Site: Hubei Provincial Maternal and Child Health Center

Country: China

Symptoms and severity: 4 of the cases were admitted with fever for investigation and 8 developed fever after childbirth. None

presented other respiratory symptoms on admission nor during hospital stay. 2 of the patients with suspected COVID-19 pneumonia reported cough, sore throat, dyspnea, diarrhea and vomiting.

Demographics: pregnant women

Age: confirmed cases: 30.9 years, suspected cases 29.8 years. Control 1:30.1 years and control 2: 29.3 years

Exposure history: none of confirmed COVID-19 patients reported an exposure history. Retrospective analysis of medical records of pregnant women with COVID-19 pneumonia and pregnant women without COVID-19 pneumonia.

Time since onset of symptoms: not reported
Whole blood. See Table 2

Clinical characteristics, laboratory test results, maternal and neonatal outcomes were collected from medical records and reviewed independently by 2 investigators Index tests were: WBC, lymphocytes, neutrophils, CRP, eosinophils, ALT, AST

\section{Target condition and reference standard(s)}

\section{Cases: RT-PCR and chest CT}

Controls: 121 women admitted during 24 January-11 February 2019 (control 2019 group)

Target condition: COVID-19

Flow and timing Blood test results were also retrieved from medical records. 2 case groups underwent blood tests every three days but 2 control groups only taken once

Comparative

Notes

Methodological quality

\begin{tabular}{llll}
\hline Item & Authors' judgement & Risk of bias & Applicability concerns \\
\hline
\end{tabular}

DOMAIN 1: Patient Selection Was a consecutive or random sample of pa- Unclear
tients enrolled?

\begin{tabular}{ll}
\hline Was a case-control design avoided? & No \\
\hline Did the study avoid inappropriate exclusions? & No \\
\hline
\end{tabular}

\author{
Could the selection of patients have intro-
duced bias?
}

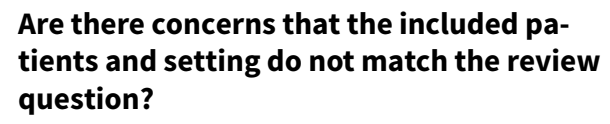

DOMAIN 2: Index Test (All tests) 
Li 2020e (Continued)

Were the index test results interpreted without knowledge of the results of the reference standard?

\begin{tabular}{l}
\hline If a threshold was used, was it pre-specified? Unclear \\
\hline $\begin{array}{l}\text { Could the conduct or interpretation of the } \\
\text { index test have introduced bias? }\end{array}$ \\
\hline $\begin{array}{l}\text { Are there concerns that the index test, its } \\
\text { conduct, or interpretation differ from the } \\
\text { review question? }\end{array}$
\end{tabular}

\section{DOMAIN 3: Reference Standard}

Is the reference standards likely to correctly Yes classify the target condition?

Were the reference standard results interpret- Unclear ed without knowledge of the results of the index tests?

Could the reference standard, its conduct,
or its interpretation have introduced bias?

Are there concerns that the target condi-
tion as defined by the reference standard
does not match the question?

\section{DOMAIN 4: Flow and Timing}

Was there an appropriate interval between in- Yes dex test and reference standard?

Did all patients receive the same reference No standard?

Were all patients included in the analysis? Yes

Could the patient flow have introduced High risk
bias?

\section{Li $2020 f$}

\section{Study characteristics}

Patient Sampling

Patient characteristics and setting
Data of this retrospective case-negative control study were collected from 105

patients first visiting the Fever Clinic of Wuhan Union Hospital from 3-7 February 2020.

Setting: hospital, emergency department, outpatient setting/fever clinic/COVID triage centre

Site: Wuhan union hospital, Wuhan

Country: China 
Symptoms and severity: $59.6 \%$ of cases had fever, $38.5 \%$ had respiratory symptoms and $1.9 \%$ had weakness compared to controls where $52.8 \%$ had fever, $47.2 \%$ had respiratory symptoms and $0 \%$ had weakness.

Demographics: cases 50\% male: controls: $56.6 \%$ male

Age: cases average years 57 years; controls average age 51 years (adults)

Exposure history: not stated

Time since onset of symptoms: not stated

Any other info:

People conducting the test, sample tested were not stated. Tests were conducted at first medical visit. Leukocyte (x 109/L; ref 3.5-9.5) normal or increased ( $\leq 3.5$ ); neutrophil (x 109/L; ref 1.8-6.3) increased; lymphocyte (x 109/L; ref 1.1-3.2) decreased (<1.1); monocytes (x 109/L; ref 0.1-0.6) increased; eosinophil ( $x 109 / \mathrm{L}$; ref 0.02-0.52) decreased; hCRP $(\mathrm{mg} / \mathrm{L} ;$ ref $<4)$ increased.

Whole blood (otherwise WBC cannot be assessed)

Target condition and reference standard(s)

Flow and timing
Nasopharyngeal swab specimens of all participants were subject to real time RT-PCR tests through amplifying ORF1ab gene and N gene of SARSCoV-2 (BioGerm, Shanghai, China)

All participants received the same reference test. Index tests were performed at participant's first medical visit. No missing data

\section{Comparative}

Notes

\section{Methodological quality}

\begin{tabular}{llll}
\hline Item & Authors' judgement & Risk of bias & $\begin{array}{l}\text { Applicability con- } \\
\text { cerns }\end{array}$
\end{tabular}

\section{DOMAIN 1: Patient Selection}

Was a consecutive or random sample of patients en- Unclear
rolled?
rolled?

\begin{tabular}{lll}
\hline Was a case-control design avoided? & Yes \\
\hline Did the study avoid inappropriate exclusions? & Unclear & \\
\hline Could the selection of patients have introduced bias? & & Unclear risk \\
\hline $\begin{array}{l}\text { Are there concerns that the included patients and set- } \\
\text { ting do not match the review question? }\end{array}$ & \\
\hline
\end{tabular}

DOMAIN 2: Index Test (All tests)

\section{Were the index test results interpreted without knowledge Unclear}

of the results of the reference standard?

If a threshold was used, was it pre-specified? Yes


Li 2020 (Continued)

Could the conduct or interpretation of the index test

Unclear risk have introduced bias?

Are there concerns that the index test, its conduct, or

Low concern interpretation differ from the review question?

\section{DOMAIN 3: Reference Standard}

Is the reference standards likely to correctly classify the Unclear target condition?

Were the reference standard results interpreted without No

knowledge of the results of the index tests?

\section{Could the reference standard, its conduct, or its inter- Unclear risk pretation have introduced bias?}

Are there concerns that the target condition as defined

High

by the reference standard does not match the ques-

tion?

\section{DOMAIN 4: Flow and Timing}

Was there an appropriate interval between index test and Unclear reference standard?

Did all patients receive the same reference standard? Yes

Were all patients included in the analysis? Unclear

\section{Study characteristics}

Patient Sampling

No inclusion criteria reported, other than patients with suspected COVID-19 viral pneumonia admitted to the infection department, emergency department, and Jinshan Branch of hospital from 22 January-17 February 2020.

Design was unclear, but study includes COVID-19 patients and patients with other viral infections.

Patient characteristics and setting

Setting: hospital, emergency department and infection department

Country: China

Symptoms and severity: unclear

Demographics: 21 male, 19 female

Age: adults; median age in diseased 46.5 (IQR 36.5-64.3), median age in non-diseased 37.5 (IQR 29.8-63.2)

Exposure history: not reported

Time since onset of symptoms: 2 (1.4) days of onset 
Li 2020 g (Continued)

Target condition and reference standard(s)
The COVID-19 group is a confirmed case, that is, the throat swab and blood 2019-nCOV nucleic acid test are positive. The non-COVID-19 group is a suspected case of COVID-19, tested negative by 2 times of pharyngeal swabs and blood 2019-nCOV nucleic acid, other viruses (influenza A/B virus or Coxsackie virus or herpes simplex virus or RSV, etc.) positive test, or imaging findings consistent with viral pneumonia

Flow and timing

No information about flow and timing

Comparative

Notes

Methodological quality

\begin{tabular}{llll}
\hline Item & $\begin{array}{l}\text { Authors' judge- } \\
\text { ment }\end{array}$ & Risk of bias & $\begin{array}{l}\text { Applicability con- } \\
\text { cerns }\end{array}$
\end{tabular}

\section{DOMAIN 1: Patient Selection}

\begin{tabular}{llll}
\hline Was a consecutive or random sample of patients enrolled? & Unclear & Unclear \\
\hline Was a case-control design avoided? & No & High risk \\
\hline Did the study avoid inappropriate exclusions? & \\
\hline Could the selection of patients have introduced bias? & High \\
\hline $\begin{array}{l}\text { Are there concerns that the included patients and setting do } \\
\text { not match the review question? }\end{array}$
\end{tabular}

\section{DOMAIN 2: Index Test (All tests)}

Were the index test results interpreted without knowledge of the Unclear
results of the reference standard?

If a threshold was used, was it pre-specified? Unclear

Could the conduct or interpretation of the index test have in- Unclear risk
troduced bias?
troduced bias?

Are there concerns that the index test, its conduct, or interpre-
tation differ from the review question?

\section{DOMAIN 3: Reference Standard}

Is the reference standards likely to correctly classify the target condition?

Were the reference standard results interpreted without knowl- Unclear edge of the results of the index tests?

\section{Could the reference standard, its conduct, or its interpretation have introduced bias?}


Li 2020g (Continued)

\section{Are there concerns that the target condition as defined by the reference standard does not match the question?}

\section{DOMAIN 4: Flow and Timing}

Was there an appropriate interval between index test and refer- Unclear ence standard?

Did all patients receive the same reference standard? Yes

Were all patients included in the analysis? Unclear

Could the patient flow have introduced bias?

Unclear risk

Liang 2020

\section{Study characteristics}

Patient Sampling

Based on epidemiological history, clinical and radiological manifestations, cases with possible or probable COVID-19 were sent for panel discussion. Paediatric patients were not included.

Patient characteristics and setting

Setting: fever clinic, pre-screened

Site: Peking University Third Hospital from 21 January-15 Febru-

ary 2020

Country: China

Symptoms and severity: on presentation, most patients (85.7\%)

had fever with a mean body temperature of 37.8 . Cough (42.9\%),

expectoration (33.3\%), fatigue (57.1\%), headache or dizziness

(38.1\%) were common symptoms. Other symptoms included shortness of breath, myalgia or arthralgia, sore throat, nasal symptoms and diarrhoea.

Demographics: male/female

Age: $24-85$ years (median 42.0, range 34.5-66)

Exposure history: imported cases from Wuhan City or Hubei Province 6 (28.6\%); known contact with individuals from Wuhan or Hubei 1 (4.8\%); known contact with cases of confirmed COVID-19 5 (23.8\%); family aggregation onset 7 (33.3\%)

Time since onset of symptoms: between 2 and 10 days

Index tests Not much information reported.

For all index tests, see Table 2

Target condition and reference standard(s)

RT-PCR. Laboratory testing of 2019-nCoV in throat swabs was performed by both Beijing Centers for Disease Control and Prevention (CDC) and Haidian District CDC. 2019-nCoV infection was target condition

Flow and timing

Comparative

Notes 
Liang 2020 (Continued)

Methodological quality

\begin{tabular}{llll}
\hline Item & $\begin{array}{l}\text { Authors' judge- } \\
\text { ment }\end{array}$ & Risk of bias & $\begin{array}{l}\text { Applicability con- } \\
\text { cerns }\end{array}$ \\
\hline
\end{tabular}

DOMAIN 1: Patient Selection

Was a consecutive or random sample of patients enrolled?

No

Was a case-control design avoided? Yes

Did the study avoid inappropriate exclusions?

No

Could the selection of patients have introduced bias?

High risk

Are there concerns that the included patients and setting do

High

not match the review question?

\section{DOMAIN 2: Index Test (All tests)}

Were the index test results interpreted without knowledge of No

the results of the reference standard?

If a threshold was used, was it pre-specified? Unclear

Could the conduct or interpretation of the index test have High risk
introduced bias?

introduced bias?

Are there concerns that the index test, its conduct, or interpretation differ from the review question?

\section{DOMAIN 3: Reference Standard}

Is the reference standards likely to correctly classify the target No condition?

Were the reference standard results interpreted without knowl- Yes edge of the results of the index tests?
Could the reference standard, its conduct, or its interpreta-
tion have introduced bias?
High risk

\section{Are there concerns that the target condition as defined by the reference standard does not match the question?}

\section{DOMAIN 4: Flow and Timing}

Was there an appropriate interval between index test and refer- Yes ence standard?

\begin{tabular}{ll}
\hline Did all patients receive the same reference standard? & No \\
\hline Were all patients included in the analysis? & Yes
\end{tabular}

\section{Could the patient flow have introduced bias?}

High risk 


\section{Study characteristics}

Patient Sampling

No sampling method reported, other than these were patients with COVID-19 in the Renmin Hospital in Wuhan from 31 January-26 February 2020

Patient characteristics and setting

\section{Setting: hospital}

Site: Renmin Hopsital of Wuhan University

Country: China

Symptoms and severity:

Demographics: cases: 55 male and 57 female; controls:

23 male and 22 female

Age: adults; mean age cases subgroups $62-63$ and cases 62 years

Exposure history: not stated

Time since onset of symptoms: not stated

Any other info:

Index tests

Urine samples, collected from catheters. All collected specimens were tested within $2 \mathrm{~h}$; no blinding reported; no timing reported, no thresholds reported

Target condition and reference standard(s)

Diagnosis and Treatment Program of New Coronavirus Pneumonia (sixth trial version); no further information on reference standard

Flow and timing

No information reported

Comparative

Notes

\section{Methodological quality}

\begin{tabular}{lll}
\hline Item & $\begin{array}{l}\text { Authors' judge- } \\
\text { ment }\end{array}$ & $\begin{array}{c}\text { Risk of bias } \\
\text { Applicability } \\
\text { concerns }\end{array}$ \\
\hline
\end{tabular}

\section{DOMAIN 1: Patient Selection}

\begin{tabular}{ll}
\hline Was a consecutive or random sample of patients enrolled? & Unclear \\
\hline Was a case-control design avoided? & No \\
\hline Did the study avoid inappropriate exclusions? & Unclear
\end{tabular}

\section{Could the selection of patients have introduced bias?}

High risk

Are there concerns that the included patients and setting do not

Unclear match the review question?

DOMAIN 2: Index Test (All tests) 
Liu 2020 (Continued)

Were the index test results interpreted without knowledge of the results of Unclear the reference standard?

If a threshold was used, was it pre-specified? Unclear

\section{Could the conduct or interpretation of the index test have introduced} bias?

\section{Are there concerns that the index test, its conduct, or interpretation} differ from the review question?

\section{DOMAIN 3: Reference Standard}

Is the reference standards likely to correctly classify the target condition? No

Were the reference standard results interpreted without knowledge of the Unclear results of the index tests?

\section{Could the reference standard, its conduct, or its interpretation have introduced bias?}

\section{Are there concerns that the target condition as defined by the refer-} ence standard does not match the question?

\section{DOMAIN 4: Flow and Timing}

Was there an appropriate interval between index test and reference stan- Unclear dard?

\begin{tabular}{ll}
\hline Did all patients receive the same reference standard? & Unclear \\
\hline Were all patients included in the analysis? & Unclear \\
\hline Could the patient flow have introduced bias? & Unclear risk
\end{tabular}

\section{Study characteristics}

Patient Sampling

Patient characteristics and setting
Hospitalized patients with confirmed or suspected

COVID-19 and at least one post-admission evaluation

\author{
Setting: hospital \\ Site: Wuhan Hankou Hospital \\ Country: China \\ Symptoms and severity: the most common signs and \\ symptoms at onset of illness were fever (323 (76.5\%)), \\ cough (258 \\ $(60.4 \%))$, and fatigue (148 (33.4\%)) \\ Demographics: median age was 55 years (IQR 39-66) \\ and $254(44.0 \%)$ were men \\ 6 days from illness onset to admission (IQR 4-9)
}

\section{Index tests}


Lu 2020 (Continued)

Index tests: WBC count, neutrophil count, lymphocyte count, prothrombin time, D-dimer, ALB, ALT, total BIL, Scr, CRP

Blinding not reported

Target condition and reference standard(s)

Diagnosis was only based on SARS-CoV-2 RT-PCR (no further information provided)

Flow and timing

Only 199/577 received RT-PCR

Time interval was unclear

\section{Comparative}

Notes

Methodological quality

\begin{tabular}{|c|c|c|c|}
\hline Item & $\begin{array}{l}\text { Authors' } \\
\text { judgement }\end{array}$ & Risk of bias & $\begin{array}{l}\text { Applicability } \\
\text { concerns }\end{array}$ \\
\hline
\end{tabular}

DOMAIN 1: Patient Selection

Was a consecutive or random sample of patients enrolled?

Yes

Was a case-control design avoided?

Yes

Did the study avoid inappropriate exclusions?

Unclear

Could the selection of patients have introduced bias?

High risk

\section{Are there concerns that the included patients and setting do not match} the review question?

\section{DOMAIN 2: Index Test (All tests)}

Were the index test results interpreted without knowledge of the results of Unclear the reference standard?

If a threshold was used, was it pre-specified? Unclear

Could the conduct or interpretation of the index test have introduced
bias?
bias?

Are there concerns that the index test, its conduct, or interpretation differ from the review question?

\section{DOMAIN 3: Reference Standard}

Is the reference standards likely to correctly classify the target condition? No

Were the reference standard results interpreted without knowledge of the Unclear results of the index tests?

Could the reference standard, its conduct, or its interpretation have introduced bias?

High risk 
Lu 2020 (Continued)

\section{Are there concerns that the target condition as defined by the reference standard does not match the question?}

\section{DOMAIN 4: Flow and Timing}

Was there an appropriate interval between index test and reference stan- Unclear dard?

Did all patients receive the same reference standard? Yes

Were all patients included in the analysis?

No

Could the patient flow have introduced bias?

Unclear risk

Mardani 2020

\section{Study characteristics}

Patient Sampling

Outpatients who presented to Behpooyan ClinicMedical center in Tehran (Iran) from 22 February-14

March 2020. with suspected COVID-19 having initial respiratory signs (including sore throat without shortness of breath), fever, cough, muscle ache, and headache were included

Patient characteristics and setting

Setting: hospital

Site: Behpooyan Clinic Medical center in Tehran

Country: Iran

Symptoms and severity: outpatients with suspected COV-

ID-19 having initial respiratory signs (including sore throat without shortness of breath), fever, cough, muscle ache, and headache were included

Demographics: 200 cases with the mean age of 41.3 , SD 14.6

(range: $19-78$ ) years were studied ( $0.53 \%$ male). $40.2 \%$ of cas-

es were in the 30-49 years age range.

Exposure history and time since onset of symptoms: not reported

Index tests

Only 2x2 table for CRP. Blood samples were collected from each participant. Whole blood

Target condition and reference standard(s)

RT-PCR for COVID-19 using pharyngeal swab samples; no information on blinding

Flow and timing

Pharyngeal swab was collected on presentation, unclear when blood samples were collected

Comparative

Notes

\section{Methodological quality}

\begin{tabular}{llll}
\hline Item & $\begin{array}{l}\text { Authors' judge- } \\
\text { ment }\end{array}$ & Risk of bias & $\begin{array}{l}\text { Applicability } \\
\text { concerns }\end{array}$
\end{tabular}

Routine laboratory testing to determine if a patient has COVID-19 (Review) 
Mardani 2020 (Continued)

DOMAIN 1: Patient Selection

\begin{tabular}{lll}
\hline Was a consecutive or random sample of patients enrolled? & Unclear & Yes \\
\hline Was a case-control design avoided? & Unclear & Unclear risk \\
\hline Did the study avoid inappropriate exclusions? & \\
\hline Could the selection of patients have introduced bias? & Unclear \\
\hline $\begin{array}{l}\text { Are there concerns that the included patients and setting do not } \\
\text { match the review question? }\end{array}$ & \\
\hline DOMAIN 2: Index Test (All tests)
\end{tabular}

Were the index test results interpreted without knowledge of the re- Unclear

sults of the reference standard?

If a threshold was used, was it pre-specified? Unclear
Could the conduct or interpretation of the index test have intro- Unclear risk duced bias?

Are there concerns that the index test, its conduct, or interpretation differ from the review question?

\section{DOMAIN 3: Reference Standard}

Is the reference standards likely to correctly classify the target condi- No tion?

Were the reference standard results interpreted without knowledge Unclear of the results of the index tests?

\section{Could the reference standard, its conduct, or its interpretation High risk have introduced bias?}

Are there concerns that the target condition as defined by the
reference standard does not match the question?

DOMAIN 4: Flow and Timing

Was there an appropriate interval between index test and reference Unclear standard?

Did all patients receive the same reference standard? Yes

Were all patients included in the analysis?

Yes

Could the patient flow have introduced bias? Unclear risk

\section{Study characteristics}


Miao 2020 (Continued)

Patient Sampling

163 consecutive adult patients with suspected COVID-19 from three tertiary hospitals in two provinces outside Hubei province

Patient characteristics and setting

Setting: tertiary hospitals

Site: 2 provinces outside Hubei province; fever emergency clinics at Shanghai General Hospital, High-tech hospital (First Affiliated Hospital of Nanchang University) and People's hospital of Yinchun City from 12 January-13 February 2020

Country: China

Symptoms and severity: suspected of COVID-19 visiting fever emergency clinics; the most common symptoms on admission were fever (49 (79.0\%)), dry cough (37 (59.7\%)), fatigue or myalgia (15 (24.2\%))

Demographics: 62 cases confirmed and 102 cases unconfirmed. Mean age confirmed group: 43.8 (SD 13.9; range 19-77); mean age unconfirmed group: 41.3 (SD 14.7; range 19-81); confirmed group 32 (51.6\%) men and non confirmed group $68(67.3 \%)$ men

Time since onset of symptoms was 7.0 (3.5-9.0) days (confirmed group) and 6.0 (4.0-9.0) days (unconfirmed group).

Compared with participants in unconfirmed group, participants in confirmed group had significantly higher proportion of Wuhan residence history, having visited Wuhan, clustering diseases and dry cough

Index tests

Target condition and reference standard(s)
WBC count, PCT, ALT, LDH, creatinine kinase, troponin I. Table 2

RT-PCR. sample: nasopharyngeal swabs or sputum specimens; the confirmed group was defined as a positive result of at least 1 RT-PCR test for SARS-CoV-2. The unconfirmed group was defined as all results of RT-PCR tests were negative

Time interval not reported; all participants received the same reference standard; no missing data or uninterpretable results

Flow and timing

\section{Comparative}

Notes

\section{Methodological quality}

\begin{tabular}{llll}
\hline Item Authors' judgement & Risk of bias & $\begin{array}{l}\text { Applicability con- } \\
\text { cerns }\end{array}$
\end{tabular}

\section{DOMAIN 1: Patient Selection}

Was a consecutive or random sample of patients en- $\quad$ Yes
rolled?
rolled?

\begin{tabular}{ll}
\hline Was a case-control design avoided? & Yes \\
\hline Did the study avoid inappropriate exclusions? & Yes \\
\hline $\begin{array}{l}\text { Could the selection of patients have introduced } \\
\text { bias? }\end{array}$ & Low risk \\
\hline
\end{tabular}


Miao 2020 (Continued)

Are there concerns that the included patients and setting do not match the review question?

\section{DOMAIN 2: Index Test (All tests)}

Were the index test results interpreted without knowl- Unclear edge of the results of the reference standard?

If a threshold was used, was it pre-specified? Unclear

Could the conduct or interpretation of the index test Unclear risk
have introduced bias?
have introduced bias?

\section{Are there concerns that the index test, its conduct,} or interpretation differ from the review question?

\section{DOMAIN 3: Reference Standard}

Is the reference standards likely to correctly classify the No

target condition?

Were the reference standard results interpreted with- Unclear out knowledge of the results of the index tests?
Could the reference standard, its conduct, or its in- terpretation have introduced bias?

\section{Are there concerns that the target condition as de- fined by the reference standard does not match the question?}

\section{DOMAIN 4: Flow and Timing}

Was there an appropriate interval between index test Unclear and reference standard?

Did all patients receive the same reference standard? Yes

Were all patients included in the analysis? Yes

Could the patient flow have introduced bias? Low risk

Pan 2020

\section{Study characteristics}

Patient Sampling

COVID-19 cases: laboratory-confirmed SARS-CoV-2 infection by real-time RT-PCR CAP cases:

- $\geq 2$ symptoms and signs of CAP and had evidence of pneumonia revealed by the emergency department physician or internal medicine consultant

- patients with a complete record of haematological and biochemical indicators 
Pan 2020 (Continued)

- hospitalized patients

Exclusion criteria:

- patients deficient in clinical haematological and biochemical data

- outpatient

Healthy controls: people who made the physical check-up in our hospital

Patient characteristics and setting
Setting: hospital

Site: Zhongnan Hospital of Wuhan University

Country: China

Symptoms and severity: patients with COVID-19 vs patients with CAP, COVID-19 patients

Demographics: median age 58 (48-70) M/F: 51/33, CAP patients: median age 71 (56-86), M/F: 142/79, healthy controls: median age 33 (24-39) M/F: 68/52

Time since onset of symptoms and exposure history not reported

Index tests

$\mathrm{Hb}$, lymphocytes, and monocytes, were analyzed. Routine serum biochemical parameters, including ALT, AST, AST/ALT ratio, total BIL, direct BIL, unconjugated BIL, total protein (TP), ALB, GLB, GGT, ALP, and total bile acid (TBA) were measured.

Cases: RT-PCR once, no further specification

Hospital controls without pneumonia, patients with CAP: not reported how confirmed

No information

Flow and timing

Comparative

Notes

Methodological quality

$\begin{array}{llll}\text { Item Authors'judgement } & \text { Risk of bias } \begin{array}{l}\text { Applicability con- } \\ \text { cerns }\end{array}\end{array}$

\section{DOMAIN 1: Patient Selection}

\begin{tabular}{lll}
\hline Was a consecutive or random sample of patients enrolled? & Unclear \\
\hline Was a case-control design avoided? & No & Unclear \\
\hline Did the study avoid inappropriate exclusions? & High risk \\
\hline Could the selection of patients have introduced bias? & \\
\hline $\begin{array}{l}\text { Are there concerns that the included patients and setting } \\
\text { do not match the review question? }\end{array}$ & \\
\hline
\end{tabular}

\section{DOMAIN 2: Index Test (All tests)}


Pan 2020 (Continued)

Were the index test results interpreted without knowledge of Unclear the results of the reference standard?

If a threshold was used, was it pre-specified? Unclear

Could the conduct or interpretation of the index test have Unclear risk
introduced bias?
introduced bias?

Are there concerns that the index test, its conduct, or interpretation differ from the review question?

\section{DOMAIN 3: Reference Standard}

Is the reference standards likely to correctly classify the target No condition?

Were the reference standard results interpreted without Unclear knowledge of the results of the index tests?
Could the reference standard, its conduct, or its interpre-
tation have introduced bias?
High risk

Are there concerns that the target condition as defined by
the reference standard does not match the question?

\section{DOMAIN 4: Flow and Timing}

Was there an appropriate interval between index test and ref- Unclear erence standard?

Did all patients receive the same reference standard? No

Were all patients included in the analysis?

No

\section{Study characteristics}

$\begin{array}{ll}\text { Patient Sampling } & \text { Those tested for COVID-19 in participants from the Veterans } \\ & \text { Affairs national Corporate Data Warehouse on members of the } \\ & \text { VA Birth Cohort from } 8 \text { February-30 March } 2020\end{array}$

Whole blood 
Rentsch 2020 (Continued)

Target condition and reference standard(s)
SARS-COV-2 assays. COVID-19 tests conducted in the VA using text searching of laboratory results 141 containing terms consistent with SARS-CoV-2 or COVID-19. If a participant had more than one test and all were negative we selected first negative, otherwise we used date of first positive. Nearly all tests utilized nasopharyngeal swabs, $1 \%$ were from other sources

All participants received same ref standard. Missings are participants for whom test are pending $(n=93)$ or inconclusive ( $n$ =33). Laboratory findings closest to baseline within a year prior or up to 1 week after baseline were used. Baseline was defined as the date of specimen collection for COVID-19 test unless testing was occurred during hospitalization, in which case it was date of admission.

\begin{tabular}{lllll}
\hline Comparative & & & \\
\hline Notes & & & \\
\hline Methodological quality & $\begin{array}{l}\text { Authors' judge- } \\
\text { ment }\end{array}$ & $\begin{array}{l}\text { Applicability con- } \\
\text { cerns }\end{array}$ & \\
\hline
\end{tabular}

DOMAIN 1: Patient Selection

\begin{tabular}{ll}
\hline Was a consecutive or random sample of patients enrolled? & Yes \\
\hline Was a case-control design avoided? & Yes \\
\hline Did the study avoid inappropriate exclusions? & Yes \\
\hline
\end{tabular}

Could the selection of patients have introduced bias?

Low risk

\section{Are there concerns that the included patients and setting do not} match the review question?

\section{DOMAIN 2: Index Test (All tests)}

Were the index test results interpreted without knowledge of the re- Unclear sults of the reference standard?

If a threshold was used, was it pre-specified? Unclear

Could the conduct or interpretation of the index test have intro- Unclear risk
duced bias?

\section{duced bias?}

Are there concerns that the index test, its conduct, or interpretation differ from the review question?

\section{DOMAIN 3: Reference Standard}

Is the reference standards likely to correctly classify the target con- Yes dition?

Were the reference standard results interpreted without knowledge Unclear of the results of the index tests? 
Rentsch 2020 (Continued)

Could the reference standard, its conduct, or its interpretation

Low risk have introduced bias?

Are there concerns that the target condition as defined by the

High reference standard does not match the question?

\section{DOMAIN 4: Flow and Timing}

Was there an appropriate interval between index test and reference No

standard?

\begin{tabular}{ll}
\hline Did all patients receive the same reference standard? & Yes \\
\hline Were all patients included in the analysis? & Unclear
\end{tabular}

Could the patient flow have introduced bias? High risk

Yang 2020b

\section{Study characteristics}

Patient Sampling

Inclusion criteria of the patients suspected of moderate type novel coronavirus pneumo-

nia for this study are:

- exposure history

- presenting with fever or respiratory symptoms, or normal or decreased WBC count at the early stage, or decreased lymphocyte count

- radiological features of novel coronavirus pneumonia

Exclusion criteria are:

- respiratory rate $\geq 30 / \mathrm{min}$

- peripheral oxygen saturation $\leq 93 \%$ when at rest

- shock

- need for mechanic ventilation or ICU care; 5. Organ failure.

In this study, the participants suspected of moderate type novel coronavirus pneumonia confirmed with positive nucleic acid tests were designated as the study group and the ones with negative findings as the control group. Duration 31 January-11 February 2020

Patient characteristics and setting

Setting: triaged for admission to the Southeast Hospital of Xiaogan Central Hospital from the fever clinics of Xiaogan Central Hospital, Xiaogan First People's Hospital and Hubei Aerospace Hospital. From 31 January-11 February 2020

Country: China

Severity: none of the participants were severely or critically ill Demographics: in cases, $51 \%$ was male and in controls $48 \%$ was male; mean age was 49.2 years $+/-13.7(95 \% \mathrm{Cl} 48-50)$

Exposure status: more than half were exposed to travellers from Wuhan

Time since onset of symptoms: mean 4.6 days from onset of symptoms (+/- 2.9$) ; 0.22 \%$ died

Index tests

The data were retrieved from the outpatient and inpatient electronic medical record system (HealthOne, Shenzhen, China), nursing records, laboratory reports and chest CT 
Yang 2020b (Continued)

scans. Laboratory findings: WBC, neutrophils, lymphocytes, Hb, platelets, CRP, PCT, ALT, AST Scr, urea, CK, CK-MB, pro-BNP, prothrombin time, INR, D-Dimer

Whole blood; thresholds not reported

Some of the routine lab tests were part of the inclusion criteria: normal or decreased WBC count at the early stage, or decreased lymphocyte count

Target condition and reference standard(s)

Pharyngeal swabs of the suspected participants were collected by a specifically trained nurse and the specimens were delivered to the central lab.

The tests were conducted with the novel coronavirus 2019-nCoV nucleic acid test kit (Shanghai ZJ BioTech, Shanghai, China) using Applied BiosystemsTM 7500 Real-Time PCR System (Thermo Fisher Scientific, USA)

Positive finding of the novel coronavirus nucleic acid test is defined as positive results with both Open reading frame $1 \mathrm{ab}(\mathrm{ORF} 1 \mathrm{ab})$ and Nucleocapsid protein $(\mathrm{N})$ for respiratory specimens examined with real-time fluorescence PCR. Negative finding of the novel coronavirus nucleic acid test is defined as 2 consecutive tests for respiratory specimens collected with intervals of at least 1 day displaying negative results as examined with real-time fluorescence PCR
Not reported

\title{
Comparative
}

\section{Notes}

\section{Methodological quality}

\begin{tabular}{llll}
\hline Item & Authors' judgement & Risk of bias & Applicability concerns \\
\hline
\end{tabular}

DOMAIN 1: Patient Selection

\begin{abstract}
Was a consecutive or random sample of Unclear patients enrolled?
\end{abstract}

\begin{tabular}{ll}
\hline Was a case-control design avoided? & Yes \\
\hline $\begin{array}{l}\text { Did the study avoid inappropriate exclu- } \\
\text { sions? }\end{array}$ & No \\
\hline
\end{tabular}

Could the selection of patients have High risk
introduced bias?

Are there concerns that the included
patients and setting do not match the
review question?

\section{DOMAIN 2: Index Test (All tests)}

Were the index test results interpreted reference standard?

\footnotetext{
If a threshold was used, was it pre-spec- Unclear ified?
} 
Yang 2020b (Continued)

Could the conduct or interpretation of the index test have introduced bias?

\section{Are there concerns that the index test, its conduct, or interpretation differ \\ from the review question?}

\section{DOMAIN 3: Reference Standard}

Is the reference standards likely to cor- Yes rectly classify the target condition?

Were the reference standard results interpreted without knowledge of the results of the index tests?

\section{Could the reference standard, its con- duct, or its interpretation have intro- duced bias?}

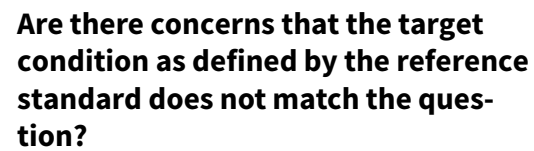

\section{DOMAIN 4: Flow and Timing}

Was there an appropriate interval be- Unclear tween index test and reference standard?

Did all patients receive the same refer- Yes ence standard?

Were all patients included in the analy- Unclear sis?

Could the patient flow have intro-
duced bias?

Yang 2020C

\section{Study characteristics}

Patient Sampling

A consecutive cohort of 73 COVID-19 and 48 influenza pneumonia patients were retrospectively recruited from 5 independent institutions

Patient characteristics and setting

COVID: 73 consecutive patients confirmed with SARSCov2, from 5 independent hospitals in 4 Chinese cities, mean age was 41.9, 41 men 32 women

Non-COVID: from 1 January 2015-30 September 2019, a total of 205 consecutive patients confirmed with influenza pneumonia from Shantou and Meizhou city were recruited. Finally, 48 influenza pneumonia patients (mean age: 40.4 years, range: $0.1-83$ years) were enrolled as controls, 
Yang 2020c (Continued)

including 30 men and 18 women; influenza $A=36$, Influenza $\mathrm{B}=12$

Index tests

Target condition and reference standard(s)

Flow and timing

Comparative

Notes
Total WBC count, lymphocyte count, lymphocyte ratio, neutrophil count, neutrophil ratio and CRP level

RT-PCR for COVID. Influenza controls were confirmed with respiratory pathogen IgM antibody test

Time interval, unclear; COVID patients, RT-PCR and influenza (IgM antibody test), missing data not noticed

\section{Methodological quality}

\begin{tabular}{|c|c|c|c|}
\hline Item & $\begin{array}{l}\text { Authors' judge- } \\
\text { ment }\end{array}$ & Risk of bias & $\begin{array}{l}\text { Applicability } \\
\text { concerns }\end{array}$ \\
\hline
\end{tabular}

\section{DOMAIN 1: Patient Selection}

\begin{tabular}{ll}
\hline Was a consecutive or random sample of patients enrolled? & Yes \\
\hline Was a case-control design avoided? & No \\
\hline Did the study avoid inappropriate exclusions? & Unclear
\end{tabular}

\begin{tabular}{ll}
\hline Could the selection of patients have introduced bias? & High risk
\end{tabular}

Are there concerns that the included patients and setting do not
match the review question?

DOMAIN 2: Index Test (All tests) Were the index test results interpreted without knowledge of the results Unclear
of the reference standard?

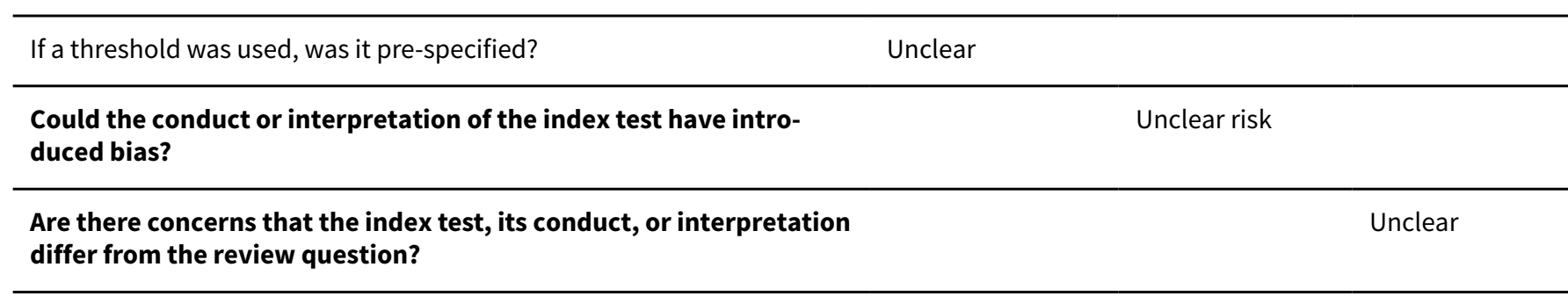

\section{DOMAIN 3: Reference Standard}

Is the reference standards likely to correctly classify the target condi- Unclear tion?

Were the reference standard results interpreted without knowledge of Unclear the results of the index tests?

\section{Could the reference standard, its conduct, or its interpretation have introduced bias?}

High risk

Routine laboratory testing to determine if a patient has COVID-19 (Review)

Copyright $\odot 2020$ The Authors. Cochrane Database of Systematic Reviews published by John Wiley \& Sons, Ltd. on behalf of The Cochrane

Collaboration. 
Yang 2020c (Continued)

\section{Are there concerns that the target condition as defined by the refer- ence standard does not match the question?}

\section{DOMAIN 4: Flow and Timing}

Was there an appropriate interval between index test and reference Unclear standard?

Did all patients receive the same reference standard? No

Were all patients included in the analysis? Yes

Could the patient flow have introduced bias?

High risk

Zhang 2020

\section{Study characteristics}

Patient Sampling

COVID-19 cases: hospitalized patients from Zhongnan Hospital of Wuhan university. COVID-19 was diagnosed based on criteria issued by the National Health Commission of China.

Controls: CAP hospitalized in Department of Respiratory and Critical Care Medicine between 22 January-22 February 2019.

5 control patients with chronic Hepatitis B or cirrhosis were excluded

Patient characteristics and setting

Setting: infectious diseases department hospital; controls in pulmonary and critical care departments

Site: Department of Infectious Disease, Zhongnan Hospital of Wuhan University

Country: China

Demographics: 4 participants $<14$ years of age; of the 115 participants in the COVID-19 group, 49 (42.60\%) were male and 66 (57.40\%) were female. Mean age at diagnosis was $49.52 \pm 17.06$ years (IQR, 35-62; range, $20-86$ years). The CAP group included 55 (48.25\%) male participants and $59(51.75 \%)$ female participants, mean age $61.11 \pm 18.84$ years (IQR, 47-76; range, 18-89 years).

Severity: 2 patients with chronic Hepatitis B were excluded, and 115 patients were included to COVID-19 group; from the controls group, four patients with Hepatitis B or cirrhosis were excluded.

Index tests Routine laboratory tests: ALT, AST, total BIL, ALP, GGT, LDH, ALB, GLB, INR, CRP

Target condition and reference standard(s)

COVID-19 was diagnosed based on criteria issued by the National Health Commission of China; includes RT-PCR once, Clinical signs and symptoms, chest CT

Controls: CAP

Flow and timing Not reported

Comparative 
Zhang 2020 (Continued)

Notes

\section{Methodological quality}

\begin{tabular}{llll}
\hline Item & Authors' judgement & Risk of bias & $\begin{array}{l}\text { Applicability con- } \\
\text { cerns }\end{array}$
\end{tabular}

DOMAIN 1: Patient Selection

Was a consecutive or random sample of patients enrolled? No

\begin{tabular}{ll}
\hline Was a case-control design avoided? & No \\
\hline Did the study avoid inappropriate exclusions? & No
\end{tabular}

Could the selection of patients have introduced bias? High risk

Are there concerns that the included patients and setting do not match the review question?

\section{DOMAIN 2: Index Test (All tests)}

Were the index test results interpreted without knowledge Unclear of the results of the reference standard?

\begin{tabular}{lll}
\hline If a threshold was used, was it pre-specified? & Unclear & \\
\hline $\begin{array}{l}\text { Could the conduct or interpretation of the index test } \\
\text { have introduced bias? }\end{array}$ & Unclear risk \\
\hline
\end{tabular}

Are there concerns that the index test, its conduct, or interpretation differ from the review question?

\section{DOMAIN 3: Reference Standard}

Is the reference standards likely to correctly classify the tar- No get condition?

Were the reference standard results interpreted without Unclear knowledge of the results of the index tests?

Could the reference standard, its conduct, or its inter-
pretation have introduced bias?

Are there concerns that the target condition as defined
by the reference standard does not match the question?

\section{DOMAIN 4: Flow and Timing}

Was there an appropriate interval between index test and Unclear reference standard?

\begin{tabular}{ll}
\hline Did all patients receive the same reference standard? & Unclear \\
\hline Were all patients included in the analysis? & Unclear
\end{tabular}

\section{Could the patient flow have introduced bias?}

Unclear risk 


\section{Study characteristics}

Patient Sampling

Study recruited 19 COVID-19 patients and 15 non-COVID-19 patients; no further information about selection criteria.

Unclear if study was a 2-gate design or a single-gate design, but the way the methods and results are described, we assumed a single-gate design.

Patient characteristics and setting

19 COVID-19 patients and 15 non-COVID-19 patients from the Second Affiliated Hospital of Anhui Medical University and Suzhou Municipal Hospital in Anhui province, China were included in this study. The mean age was 48 (IQR 27 56) and 35 (IQR 27 46) in COVID-19 and non-COVID-19 patients, respectively. 8 (42.11\%) were female in COVID-19 patients, and $9(60 \%)$ in non-COVID-19 patients. The median duration from exposure to onset is 8 (IQR 6 11) and 5 (IQR 4 11) days in COVID-19 and non-COVID-19 patients, respectively. All participants had a history of exposure to confirmed case of 2019-nCoV or travel to Hubei before illness

Index tests

Index tests done: WBC and lymphocyte count, neutrophil count, AST; ALT; LDH; GGT; $\alpha$-hydroxybutyric dehydrogenase; CK; CRP and IL-6. Tests were done on admission (4-5 days from onset), person doing the testing is not stated.

As WBC was assessed, sample must have been whole blood

\section{Target condition and reference standard(s)}

COVID-19 cases were confirmed to be infected with or without 2019-nCoV by real-time RT-PCR. COVID-19 was defined to be 2019-nCoV negative by PCR detection. For non-COVID-19 confirmation, we collected a throat swab or sputum sampling every other day.

The patient was confirmed as non-COVID-19 if 3 consecutive real-time PCR tests were negative during first 7 days of admission

Flow and timing

All participants received the same reference test. Test interval is 4-5 days. No missing data.

Index tests were performed at admission. it is not clear when the reference test was done.

\section{Comparative}

$$
\text { Notes }
$$

Methodological quality

\begin{tabular}{|c|c|c|c|}
\hline Item & Authors' judgement & Risk of bias & $\begin{array}{l}\text { Applicability con- } \\
\text { cerns }\end{array}$ \\
\hline \multicolumn{4}{|l|}{ DOMAIN 1: Patient Selection } \\
\hline $\begin{array}{l}\text { Was a consecutive or random sample of patients en- } \\
\text { rolled? }\end{array}$ & Yes & & \\
\hline Was a case-control design avoided? & Yes & & \\
\hline Did the study avoid inappropriate exclusions? & Unclear & & \\
\hline
\end{tabular}


Zhao 2020 (Continued)

Could the selection of patients have introduced bias?

Are there concerns that the included patients and setting do not match the review question?

\section{DOMAIN 2: Index Test (All tests)}

Were the index test results interpreted without knowledge of the results of the reference standard?

Unclear

If a threshold was used, was it pre-specified? Yes

Could the conduct or interpretation of the index $\quad$ Unclear risk
test have introduced bias?

Are there concerns that the index test, its conduct, or interpretation differ from the review

Low concern question?

\section{DOMAIN 3: Reference Standard}

Is the reference standards likely to correctly classify Yes the target condition?

Were the reference standard results interpreted Unclear without knowledge of the results of the index tests?

Could the reference standard, its conduct, or its $\quad$ Unclear risk
interpretation have introduced bias?
interpretation have introduced bias?

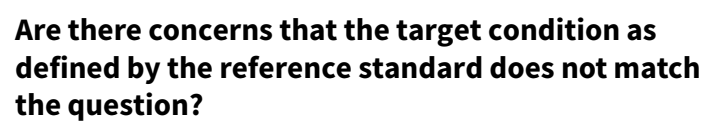

\section{DOMAIN 4: Flow and Timing}

Was there an appropriate interval between index Unclear
test and reference standard?

Did all patients receive the same reference stan- Yes dard?
Were all patients included in the analysis?
Unclear

\section{Could the patient flow have introduced bias?}

Unclear risk

\section{Zhu 2020}

\section{Study characteristics}

Patient Sampling

The inclusion criteria were

- patients defined as suspected SARS-CoV-2 infection based on guidelines for the diagnosis and treatment of pneumonia caused by novel coronavirus infection (trial version III)

- presentation to, clinical observation and quarantine in our emergency department and 
The exclusion criteria were

- transfer from another hospital or previous visit to our hospital

- previous diagnosis of COVID-19

Inclusion period between 24 January 2020 and 20 February 2020

Patient characteristics and setting
Setting: hospital, emergency department and infectious diseases satellite hospital

Site: The First Affiliated Hospital of University of Science and Technology of China, Hefei

Country: China

Symptoms and severity: there were $6(19 \%)$ smokers among diagnosed participants and $13(15 \%)$ among negative cases. 7 (22\%) diagnosed and $15(18 \%)$ negative cases had hypertension. There were no other commonly found comorbidities in either group.

Demographics: median age 40 (IQR 27-53); 46\% male

Exposure history: there was no specific exposure history common to all participants with suspected disease: $8(25 \%)$ diagnosed participants had visited Wuhan in the previous 2 weeks and 12 (38\%) had been exposed to participants with infection in the previous 2 weeks. In negative cases, these numbers were $7(20 \%)$ and $8(24 \%)$, respectively. None of the participants had a history of exposure to the seafood market in Wuhan.

Time since onset of symptoms: median 5 days (IQR 2-7 days)
Clinical and laboratory data on admission were obtained from detailed medical records, collected in a standardized case report form by 2 experienced emergency doctors. Laboratory tests included a complete blood count, serum biochemistry, IL-6 test, CK test, LDH test, and tests for the identification of other respiratory pathogens

Timing of tests not reported; blinding not reported
Target condition and reference standard(s)
A nucleic acid amplification test was performed on swab specimens from participants with suspected disease at admission. Participants with a positive diagnosis were admitted to the hospital, while participants with a negative initial result were kept in quarantine and underwent a second nucleic acid test after $24 \mathrm{~h}$; of these, participants with a second negative result on the nucleic acid test were considered to not have an infection and were discharged from the hospital once they tested negative for SARS-CoV-2 antigens on 2 consecutive tests.

Flow and timing Exact timing of lab tests was not reported.

Quote: "Not all patients presented at the same infection stage and some data were missing; thus, data could not be integrated."

Comparative

Notes

\section{Methodological quality}

Item Authors' judgement $\quad$ Risk of bias $\quad$ Applicability concerns

\section{DOMAIN 1: Patient Selection}

Was a consecutive or random sample of Unclear patients enrolled? 
Zhu 2020 (Continued)

Was a case-control design avoided? Yes

Did the study avoid inappropriate exclu- Yes sions?

Could the selection of patients have Unclear risk
introduced bias?

introduced bias?

Are there concerns that the included

Low concern patients and setting do not match the review question?

\section{DOMAIN 2: Index Test (All tests)}

Were the index test results interpreted Unclear without knowledge of the results of the reference standard?

If a threshold was used, was it pre-spec- Unclear ified?

Could the conduct or interpretation of

Unclear risk

the index test have introduced bias?

Are there concerns that the index test,

Low concern

its conduct, or interpretation differ

from the review question?

\section{DOMAIN 3: Reference Standard}

Is the reference standards likely to cor- Yes

rectly classify the target condition?

Were the reference standard results in- Yes

terpreted without knowledge of the re-

sults of the index tests?

\section{Could the reference standard, its con-}

duct, or its interpretation have intro-

duced bias?

\section{Are there concerns that the target condition as defined by the reference \\ standard does not match the ques- \\ tion?}

\section{DOMAIN 4: Flow and Timing}

Was there an appropriate interval be- Unclear tween index test and reference standard?

Did all patients receive the same refer- Yes ence standard?

Were all patients included in the analy- Unclear sis? 
Zhu 2020 (Continued)

\section{Could the patient flow have intro- duced bias? \\ Unclear risk}

ALB: albumin; ALP: alkaline phosphatase; ALT: alanine aminotransferase; ARS: acute respiratory syndrome; AST: aspartate aminotransferase; BIL: bilirubin; BNP: B-type natriuretic peptide; CAP: community-acquired pneumonia; CI: confidence interval; CK: creatine kinase; CK-MB: creatine kinase (blood); CRP: C-reactive protein; CT: computed tomography; ESR: erythrocyte sedimentation rate; GGT: $\gamma$-glutamyl transpeptidase; GLB: globulin; Hb: haemoglobin; ICU: intensive care unit; IFN-y: interferon gamma; IL: interleukin; INR: international normalized ratio; IQR: interquartile range; LDH: lactate hydrogenase; PCR: polymerase chain reaction; PCT: procalcitonin; RNA: ribonucleic acid; (r)RT-PCR: (rapid) reverse-transcriptase polymerase chain reaction; RSV: respiratory syncytial virus; Scr: serum creatinine; SD: standard deviation; WBC: white blood cell; WHO: World Health Organization;

Characteristics of excluded studies [ordered by study ID]

\begin{tabular}{|c|c|}
\hline Study & Reason for exclusion \\
\hline Ai 2020a & Insufficient data for $2 \times 2$ table \\
\hline Chen 2020a & Insufficient data for $2 \times 2$ table \\
\hline Chen 2020b & Insufficient data for $2 \times 2$ table + target condition not clear \\
\hline Cheng 2020 & Insufficient data for $2 \times 2$ table \\
\hline Giamarellos 2020 & Insufficient data for $2 \times 2$ table \\
\hline Han 2020 & Insufficient data for $2 \times 2$ table \\
\hline Kurstjens 2020 & Insufficient data for $2 \times 2$ table \\
\hline Li 2020a & Insufficient data for $2 \times 2$ table + Hospital discharge versus no discharge \\
\hline Li 2020b & Insufficient data for $2 \times 2$ table + Mechanical ventilation versus no mechanical ventilation \\
\hline Li $2020 \mathrm{C}$ & Insufficient data for $2 \times 2$ table + RNA positive versus RNA negative \\
\hline Ling 2020 & Insufficient data for $2 \times 2$ table \\
\hline Meng 2020 & Insufficient data for $2 \times 2$ table \\
\hline Peng 2020 & Insufficient data for $2 \times 2$ table \\
\hline Peng 2020a & Insufficient data for $2 \times 2$ table \\
\hline Shi 2020 & Insufficient data for $2 \times 2$ table \\
\hline Song 2020 & Insufficient data for $2 \times 2$ table \\
\hline Spiezia 2020 & Insufficient data for $2 \times 2$ table \\
\hline Sun 2020 & Insufficient data for $2 \times 2$ table \\
\hline Tang 2020 & Insufficient data for $2 \times 2$ table \\
\hline Wang 2020 & Insufficient data for $2 \times 2$ table + diagnostic prediction model \\
\hline
\end{tabular}




\begin{tabular}{ll}
\hline Study & Reason for exclusion \\
\hline Wu 2020 & Insufficient data for $2 \times 2$ table + diagnostic artificial intelligence model \\
\hline Xu 2020 & Insufficient data for $2 \times 2$ table \\
\hline Yang $2020 a$ & Insufficient data for $2 \times 2$ table \\
\hline Yin 2020 & Insufficient data for $2 \times 2$ table \\
\hline
\end{tabular}

\section{A T A}

Presented below are all the data for all of the tests entered into the review.

\section{Table Tests. Data tables by test}

\begin{tabular}{|c|c|c|}
\hline Test & No. of studies & No. of participants \\
\hline 1 WBC increase & 15 & 5318 \\
\hline 2 WBC decrease & 11 & 5111 \\
\hline 3 Leukocyturia & 1 & 164 \\
\hline 4 Monocyte count increase & 4 & 686 \\
\hline 5 Monocyte count decrease & 2 & 620 \\
\hline 6 Monocyte percentage increase & 1 & 26 \\
\hline 7 Neutrophil count increase & 11 & 1838 \\
\hline 8 Neutrophil count decrease & 4 & 734 \\
\hline 9 Neutrophil percentage increase & 4 & 283 \\
\hline 10 Neutrophil Percentage decrease & 1 & 26 \\
\hline 11 Lymphocyte count increase & 3 & 647 \\
\hline 12 Lymphocyte count decrease & 13 & 4965 \\
\hline 13 Lymphocyte percentage increase & 1 & 26 \\
\hline 14 Lymphocyte percentage decrease & 4 & 367 \\
\hline 15 Eosinophil count increase & 3 & 371 \\
\hline 16 Eosinophil count decrease & 2 & 410 \\
\hline 17 Eosinophil percentage increase & 1 & 26 \\
\hline 18 Basophil count increase & 2 & 331 \\
\hline
\end{tabular}

Routine laboratory testing to determine if a patient has COVID-19 (Review) 


\begin{tabular}{|c|c|c|}
\hline Test & No. of studies & No. of participants \\
\hline 19 Basophil percentage increase & 1 & 26 \\
\hline 20 Red Blood Cell volume distribution increase & 2 & 331 \\
\hline 21 RBC decrease & 2 & 331 \\
\hline 22 Platelets decreased & 4 & 4171 \\
\hline 23 Haemoglobin (HGB) Decreased & 3 & 3675 \\
\hline 24 Serum creatinine increased & 4 & 4316 \\
\hline 25 Creatine Kinase - increase & 5 & 1073 \\
\hline 26 Creatine Kinase MB - increase & 2 & 773 \\
\hline 27 Urea increase & 2 & 569 \\
\hline 28 ALT increase & 9 & 5162 \\
\hline 29 AST increase & 7 & 4891 \\
\hline 30 Total bilirubin (TBIL) increase & 4 & 771 \\
\hline 31 Erythrocyte Sedimentation Rate (ESR) increase & 2 & 395 \\
\hline 32 CRP increase & 14 & 2281 \\
\hline $33 \mathrm{a}-\mathrm{HBDH}$ increased & 2 & 327 \\
\hline $34 \mathrm{HCT}$ increased & 1 & 26 \\
\hline 35 HCT decreased & 2 & 331 \\
\hline 36 Albumin (ALB) decreased & 4 & 4072 \\
\hline 37 Globulin (GLB) increase & 2 & 534 \\
\hline 38 Globulin (GLB) decrease & 1 & 305 \\
\hline 39 Procalcitonin (PCT) increase & 6 & 1345 \\
\hline 40 eGFR & 1 & 3621 \\
\hline 41 Proteinuria & 1 & 164 \\
\hline 42 Prothrombin time (PT) increase & 2 & 555 \\
\hline 43 GGT increased & 3 & 566 \\
\hline $44 \mathrm{D}$-dimer increase & 3 & 659 \\
\hline $45 \mathrm{IL}-2$ & 1 & 56 \\
\hline $46 \mathrm{IL}-4$ & 1 & 56 \\
\hline
\end{tabular}




\begin{tabular}{|c|c|c|}
\hline Test & No. of studies & No. of participants \\
\hline 47 Interleukin-6 (IL-6) increase & 4 & 216 \\
\hline $48 \mathrm{IL}-8$ & 1 & 56 \\
\hline 49 IL-10 & 1 & 56 \\
\hline 50 TNF alpha & 1 & 56 \\
\hline 51 ALP increased & 2 & 534 \\
\hline 52 pro-BNP & 1 & 380 \\
\hline 53 Hematuria & 1 & 164 \\
\hline 54 INR increase & 2 & 658 \\
\hline $55 \mathrm{LDH}$ increase & 5 & 813 \\
\hline 56 Mean corpuscular volume increase & 1 & 305 \\
\hline 57 Mean corpuscular volume decrease & 1 & 305 \\
\hline 58 Erythrocyte mean corpuscular hemoglobin increase & 1 & 305 \\
\hline 59 Erythrocyte mean corpuscular hemoglobin decrease & 1 & 305 \\
\hline 60 Erythrocytemean corpuscular hemoglobin concentrate increase & 1 & 305 \\
\hline 61 Erythrocytemean corpuscular hemoglobin concentrate decrease & 1 & 305 \\
\hline 62 Mean Platelet Volume & 1 & 305 \\
\hline 63 Direct bilirubin & 1 & 305 \\
\hline 64 unconjugated bilirubin & 1 & 305 \\
\hline 65 Total protein & 1 & 305 \\
\hline 66 Total bile acid & 1 & 305 \\
\hline 67 Troponin I & 1 & 163 \\
\hline
\end{tabular}




\section{Test 1. WBC increase}

WBC increase

\begin{tabular}{|c|c|c|c|c|c|c|c|c|}
\hline Study & TP & FP & FN & TN & Sensitivity $(95 \% \mathrm{CI})$ & Specificity $(95 \% \mathrm{Cl})$ & Sensitivity $(95 \%$ & ecificity $(95$ \\
\hline Ai $2020 \mathrm{~b}$ & 2 & 18 & 106 & 189 & $0.02[0.00,0.07]$ & $0.91[0.87,0.95]$ & 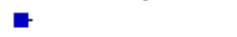 & \\
\hline Chen $2020 \mathrm{c}$ & 5 & 12 & 73 & 14 & $0.06[0.02,0.14]$ & $0.54[0.33,0.73]$ & & \\
\hline Feng 2020 & 0 & 3 & 7 & 16 & $0.00[0.00,0.41]$ & $0.84[0.60,0.97]$ & & \\
\hline Ferrari 2020 & 12 & 40 & 93 & 62 & $0.11[0.06,0.19]$ & $0.61[0.51,0.70]$ & & - \\
\hline Hsih 2020 & 0 & 6 & 2 & 35 & $0.00[0.00,0.84]$ & $0.85[0.71,0.94]$ & & \\
\hline Li $2020 f$ & 2 & 4 & 50 & 49 & $0.04[0.00,0.13]$ & $0.92[0.82,0.98]$ & $=$ & \\
\hline Li $2020 \mathrm{~g}$ & 0 & 5 & 10 & 25 & $0.00[0.00,0.31]$ & $0.83[0.65,0.94]$ & - & \\
\hline Liang 2020 & 0 & 14 & 21 & 53 & $0.00[0.00,0.16]$ & $0.79[0.67,0.88]$ & $=$ & \\
\hline Lu 2020 & 5 & 7 & 51 & 87 & $0.09[0.03,0.20]$ & $0.93[0.85,0.97]$ & $=-$ & \\
\hline Miao 2020 & 4 & 15 & 58 & 86 & $0.06[0.02,0.16]$ & $0.85[0.77,0.91]$ & & \\
\hline Pan 2020 & 9 & 76 & 75 & 145 & $0.11[0.05,0.19]$ & $0.66[0.59,0.72]$ & - & - \\
\hline Rentsch 2020 & 72 & 119 & 481 & 2829 & $0.13[0.10,0.16]$ & $0.96[0.95,0.97]$ & 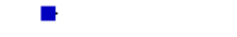 & \\
\hline Yang $2020 \mathrm{c}$ & 53 & 11 & 20 & 37 & $0.73[0.61,0.82]$ & $0.77[0.63,0.88]$ & $\rightarrow-$ & $\rightarrow-$ \\
\hline Zhao 2020 & 0 & 2 & 19 & 13 & $0.00[0.00,0.18]$ & $0.87[0.60,0.98]$ & $=$ & \\
\hline Zhu 2020 & 1 & 6 & 31 & 78 & $0.03[0.00,0.16]$ & $0.93[0.85,0.97]$ & & 7 \\
\hline
\end{tabular}

Test 2. WBC decrease

WBC decrease

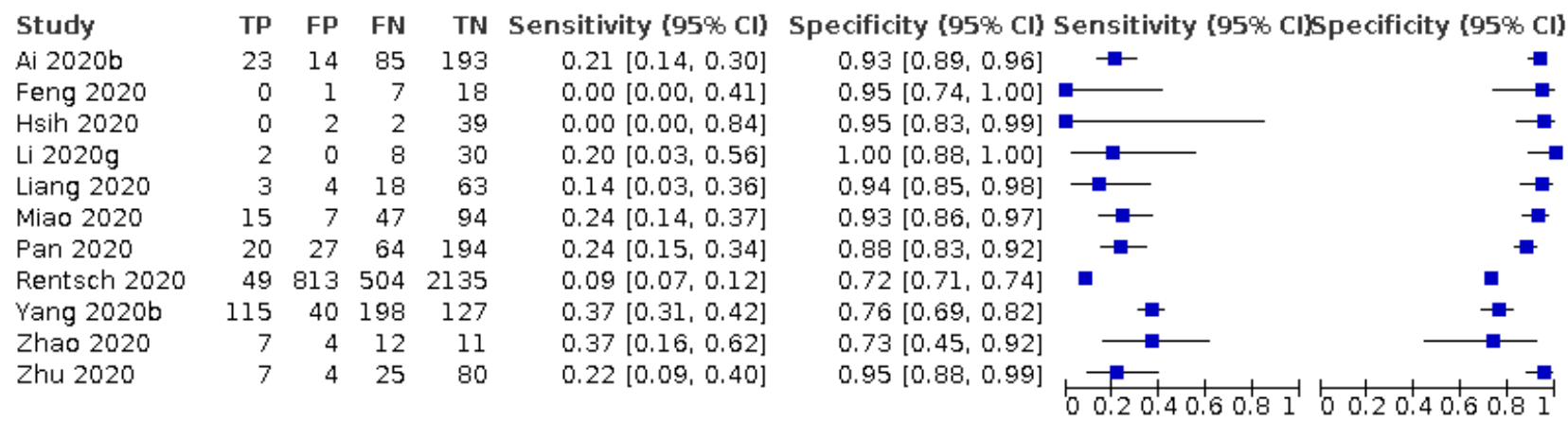

Test 3. Leukocyturia

Leukocyturia

Study TP FP FN TN Sensitivity $(95 \% \mathrm{CI})$ Specificity $(95 \% \mathrm{Cl})$ Sensitivity $(95 \% \mathrm{Cl})$ Specificity $(95 \% \mathrm{Cl})$ Liu $2020 \quad 18 \quad 5 \quad 101 \quad 40 \quad 0.15[0.09,0.23] \quad 0.89[0.76,0.96]$

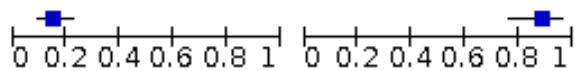

Test 4. Monocyte count increase

Monocyte count increase

$\begin{array}{lrrrrrr}\text { Study } & \text { TP } & \text { FP } & \text { FN } & \text { TN } & \text { Sensitivity }(95 \% \mathrm{Cl}) & \text { Specificity }(95 \% \mathrm{Cl}) \text { Sensitivity }(95 \% \text { Cl]Specificity (95\% Cl) } \\ \text { Ai } 2020 \mathrm{~b} & 15 & 48 & 93 & 159 & 0.14[0.08,0.22] & 0.77[0.70,0.82] \\ \text { Feng } 2020 & 1 & 2 & 6 & 17 & 0.14[0.00,0.58] & 0.89[0.67,0.99] \\ \text { Li } 2020 \mathrm{~g} & 1 & 9 & 9 & 21 & 0.10[0.00,0.45] & 0.70[0.51,0.85]- \\ \text { Pan } 2020 & 10 & 97 & 74 & 124 & 0.12[0.06,0.21] & 0.56[0.49,0.63]\end{array}$




\section{Test 5. Monocyte count decrease}

Monocyte count decrease

\begin{tabular}{|c|c|c|c|c|c|c|c|}
\hline Study & TP & FP & FN & TN & Sensitivity $(95 \% \mathrm{Cl})$ & Specificity $(95 \% \mathrm{CI})$ & Sensitivity $(95 \%$ Cl)Specificity $(95 \% \mathrm{Cl})$ \\
\hline Ai $2020 \mathrm{~b}$ & 2 & 6 & 106 & 201 & $0.02[0.00,0.07]$ & $0.97[0.94,0.99]$ & 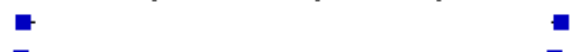 \\
\hline Pan 2020 & 1 & 13 & 83 & 208 & $0.01[0.00,0.06]$ & $0.94[0.90,0.97]$ & $P$ \\
\hline
\end{tabular}

Test 6. Monocyte percentage increase

Monocyte percentage increase

Study TP FP FN TN Sensitivity (95\% Cl) Specificity (95\% Cl) Sensitivity (95\% Cl)Specificity (95\% Cl) Feng $2020 \quad 4 \quad 8 \quad 3 \quad 11 \quad 0.57[0.18,0.90] \quad 0.58[0.33,0.80] \stackrel{1}{1} \quad \begin{array}{llll}1 & 1\end{array}$

\section{Test 7. Neutrophil count increase}

Neutrophil count increase

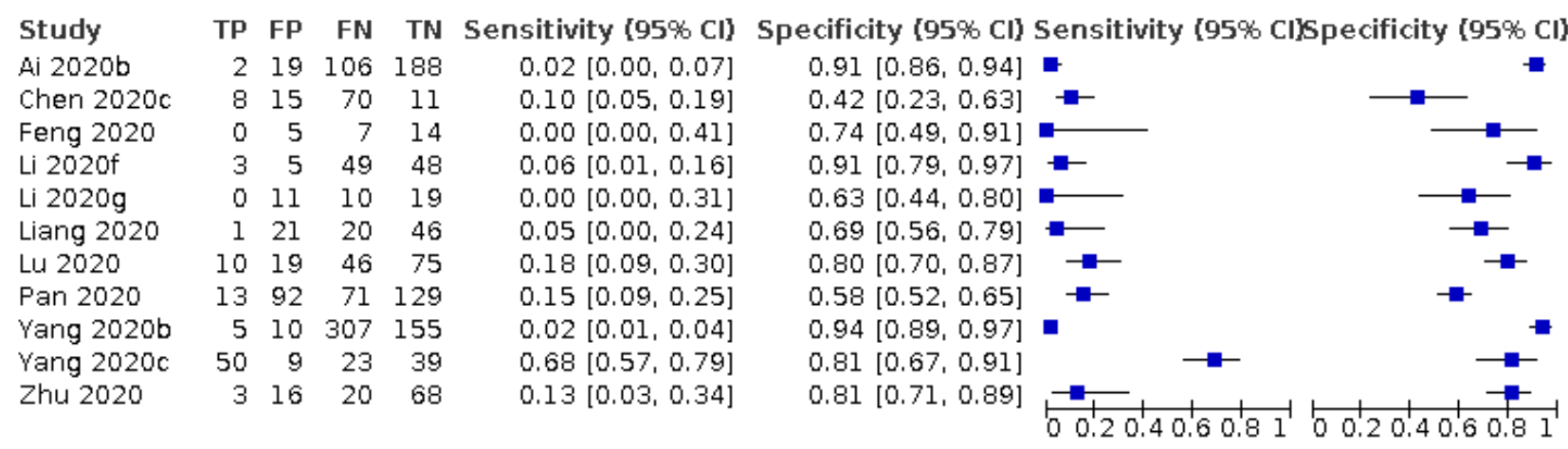

Test 8. Neutrophil count decrease

Neutrophil count decrease

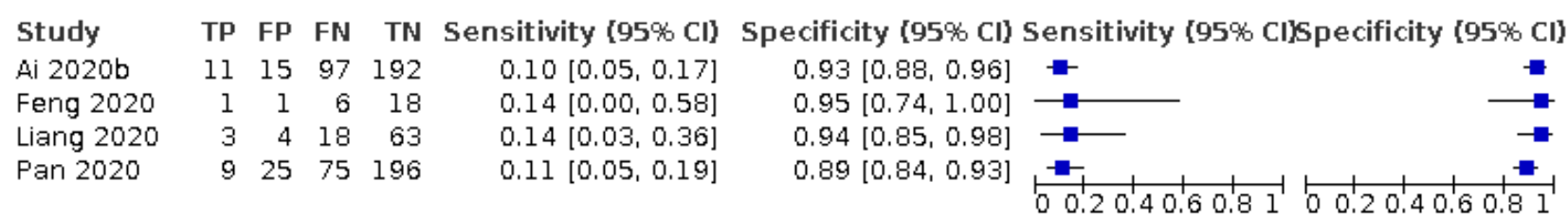




\section{Test 9. Neutrophil percentage increase}

Neutrophil percentage increase

Study TP FP FN TN Sensitivity $(95 \% \mathrm{Cl})$ Specificity $(95 \% \mathrm{Cl})$ Sensitivity $[95 \% \mathrm{Cl})$ Specificity $(95 \% \mathrm{Cl})$ $\begin{array}{llllllll}\text { Chen } 2020 \mathrm{c} & 16 & 16 & 62 & 10 & 0.21 & {[0.12,0.31]} & 0.38 \\ {[0.20,0.59]}\end{array}$ Feng $2020 \quad 1 \quad 12 \quad 6 \quad 7 \quad 0.14[0.00,0.58]$ $\begin{array}{lllllll}\text { Vang } 2020 \mathrm{c} & 50 & 17 & 23 & 31 & 0.68[0.57,0.79]\end{array}$

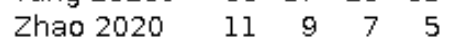

$0.61[0.36,0.83]$

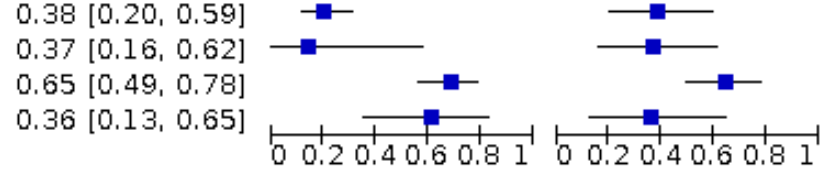

Test 10. Neutrophil Percentage decrease

Neutrophil Percentage decrease

Study TP FP FN TN Sensitivity $[95 \% \mathrm{Cl}$ ) Specificity (95\% Cl) Sensitivity $(95 \%$ Cl)Specificity (95\% Cl)

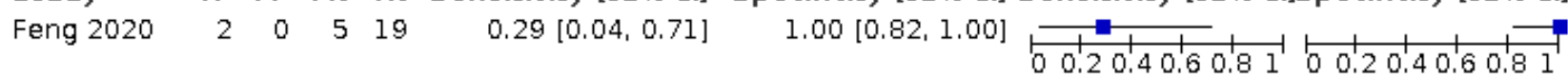

Test 11. Lymphocyte count increase

Lymphocyte count increase

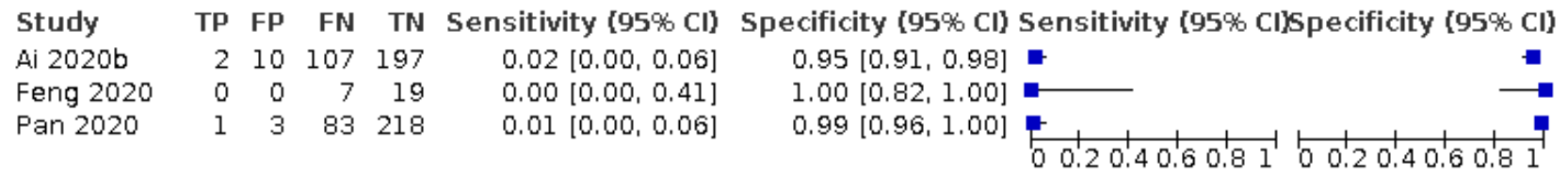

Test 12. Lymphocyte count decrease

Lymphocyte count decrease

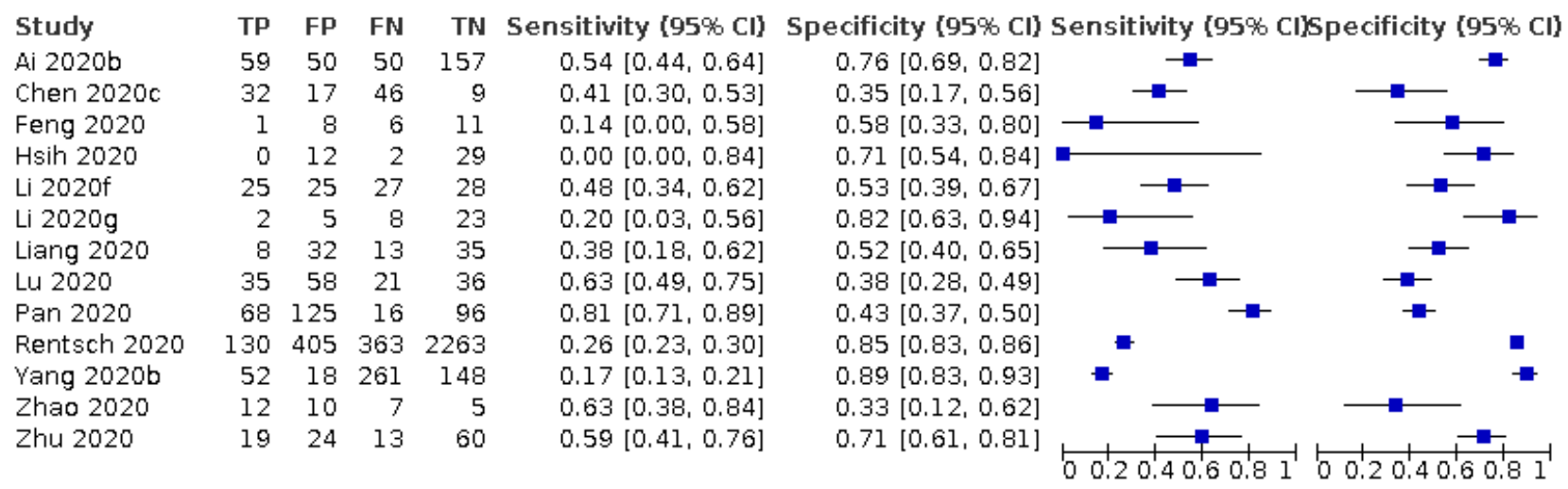




\section{Test 13. Lymphocyte percentage increase}

Lymphocyte percentage increase

Study TP FP FN TN Sensitivity $[95 \% \mathrm{Cl})$ Specificity $(95 \% \mathrm{Cl})$ Sensitivity $[95 \% \mathrm{Cl}$ Specificity $[95 \% \mathrm{Cl})$ Feng $2020 \quad 1 \quad 0 \quad 6 \quad 19 \quad 0.14[0.00,0.58] \quad 1.00[0.82,1.00]$

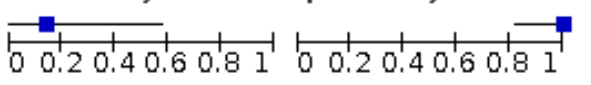

Test 14. Lymphocyte percentage decrease

Lymphocyte percentage decrease

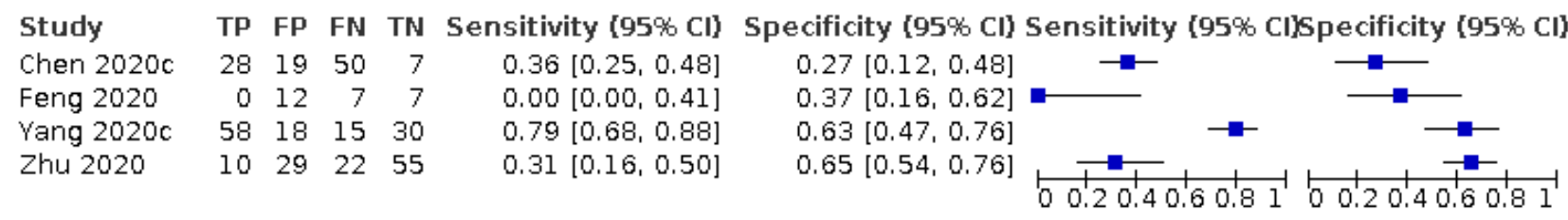

Test 15. Eosinophil count increase

Eosinophil count increase

Study TP FP FN TN Sensitivity $(95 \% \mathrm{Cl})$ Specificity $(95 \% \mathrm{Cl})$ Sensitivity $(95 \% \mathrm{Cl}) \mathrm{Specificity} \mathrm{(95 \% \textrm {Cl } )}$ Feng $2020 \quad 0 \quad 007619 \quad 0.00[0.00,0.41] \quad 1.00[0.82,1.00]$

Li $2020 \mathrm{~g} \quad 0 \quad 1 \quad 10 \quad 29 \quad 0.00[0.00,0.31]$

$0.97[0.83,1.00]$

Pan 2020

$\begin{array}{llll}0 & 6 & 84 & 215\end{array}$

$0.00[0.00,0.04]$

$0.97[0.94,0.99]$

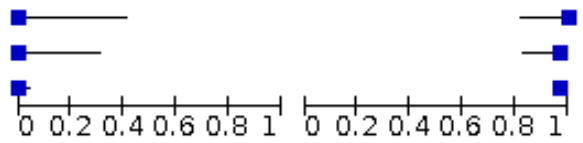

Test 16. Eosinophil count decrease

Eosinophil count decrease

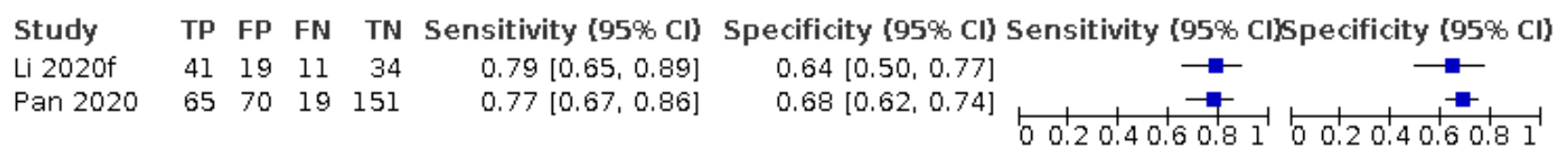

Test 17. Eosinophil percentage increase

Eosinophil percentage increase

Study TP FP FN TN Sensitivity $(95 \% \mathrm{Cl})$ Specificity $(95 \% \mathrm{Cl})$ Sensitivity $(95 \% \mathrm{Cl})$ Specificity $(95 \% \mathrm{Cl})$ Feng $2020 \quad 0 \quad 00719 \quad 0.00[0.00,0.41] \quad 1.00[0.82,1.00]$

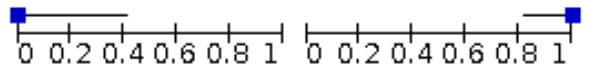


Test 18. Basophil count increase

Basophil count increase

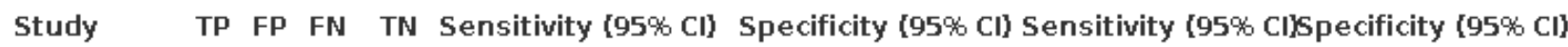
Feng $2020 \quad 0 \quad 007 \quad 79 \quad 0.00[0.00,0.41] \quad 1.00[0.82,1.00]$ $\left.\begin{array}{lllllll}\text { Pan } 2020 & 0 & 44 & 84 & 177 & 0.00 & {[0.00,0.04}\end{array}\right] \quad 0.80[0.74,0.85]$

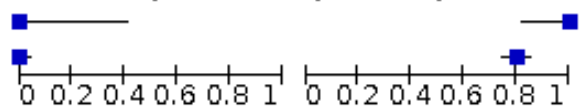

Test 19. Basophil percentage increase

Basophil percentage increase

Study TP FP FN TN Sensitivity $(95 \% \mathrm{Cl})$ Specificity $(95 \% \mathrm{Cl})$ Sensitivity $(95 \% \mathrm{Cl})$ Specificity (95\% Cl) Feng $2020 \quad 1 \quad 0 \quad 6 \quad 19 \quad 0.14[0.00,0.58] \quad 1.00[0.82,1.00]$

\section{Test 20. Red Blood Cell volume distribution increase}

Red Blood Cell volume distribution increase

Study TP FP FN TN Sensitivity $(95 \% \mathrm{Cl})$ Specificity $(95 \% \mathrm{Cl})$ Sensitivity $(95 \% \mathrm{Cl}) \mathrm{Specificity} \mathrm{(95 \% \textrm {Cl } )}$

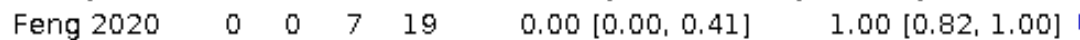

$\begin{array}{lllllll}\text { Pan } 2020 & 3 & 65 & 81 & 156 & 0.04[0.01,0.10 & 0.71[0.64,0.77]\end{array}$

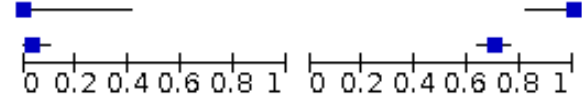

Test 21. RBC decrease

RBC decrease

Study TP FP FN TN Sensitivity $(95 \% \mathrm{Cl})$ Specificity $(95 \% \mathrm{Cl})$ Sensitivity $(95 \% \mathrm{Cl})$ Specificity (95\% Cl)

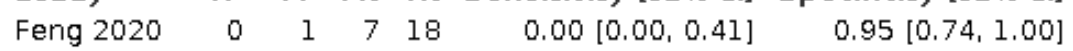

$\begin{array}{llllllll}\text { Pan } 2020 & 33 & 185 & 51 & 36 & 0.39 & {[0.29,0.51]} & 0.16\end{array}[0.12,0.22]$

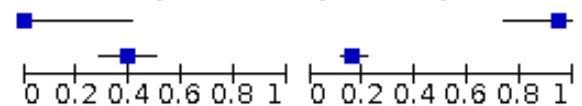

Test 22. Platelets decreased

Platelets decreased

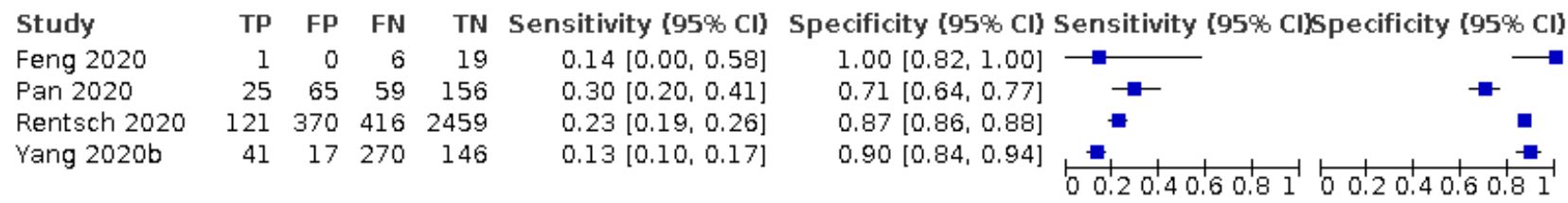


Test 23. Haemoglobin (HGB) Decreased

Haemoglobin (HGB) Decreased

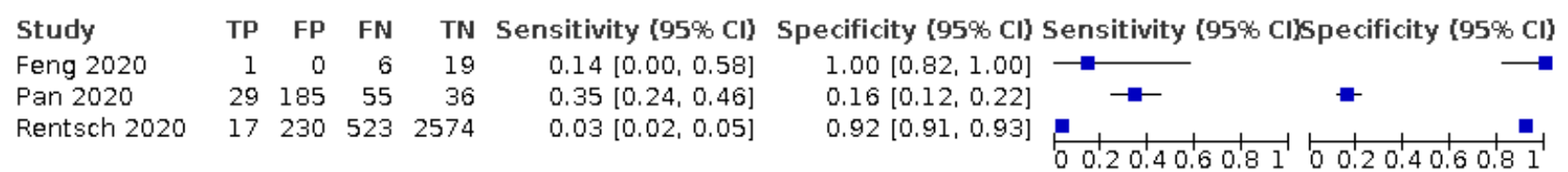

\section{Test 24. Serum creatinine increased}

Serum creatinine increased

$\begin{array}{lrrrrrr}\text { Study } & \text { TP } & \text { FP } & \text { FN } & \text { TN } & \text { Sensitivity }(95 \% \mathrm{Cl}) & \text { Specificity }(95 \% \mathrm{Cl}) \text { Sensitivity }(95 \% \mathrm{Cl}] \mathrm{Specificity}(95 \% \mathrm{Cl}) \\ \text { Chen } 2020 \mathrm{C} & 11 & 14 & 67 & 12 & 0.14[0.07,0.24] & 0.46[0.27,0.67]- \\ \text { Lu 2020 } & 1 & 3 & 53 & 84 & 0.02[0.00,0.10] & 0.97[0.90,0.99] \\ \text { Rentsch 2020 } & 130 & 440 & 435 & 2598 & 0.23[0.20,0.27] & 0.86[0.84,0.87]\end{array}$

\section{Test 25. Creatine Kinase - increase}

Creatine Kinase - increase

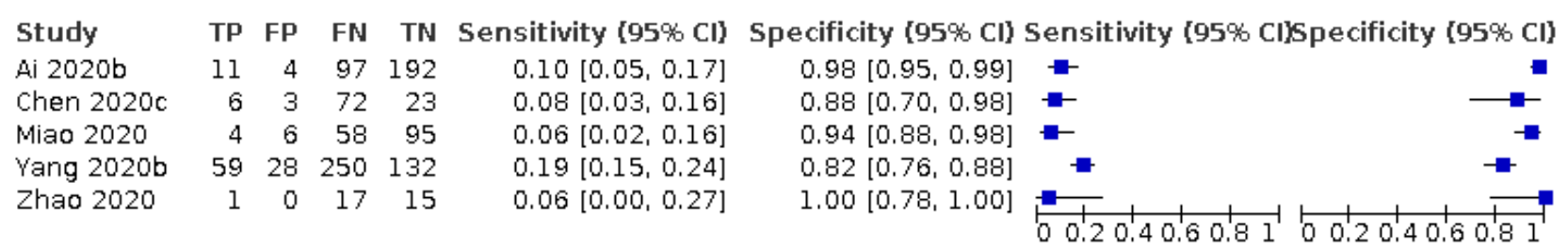

\section{Test 26. Creatine Kinase MB - increase}

Creatine Kinase MB - increase

\begin{tabular}{|c|c|c|c|c|c|c|c|}
\hline Study & TP & FP & FN & TN & Sensitivity $(95 \% \mathrm{Cl})$ & Specificity $(95 \% \mathrm{Cl})$ & Sensitivity $(95 \%$ Cl)Specificity $(95 \% \mathrm{Cl})$ \\
\hline Ai $2020 \mathrm{~b}$ & 10 & 27 & 98 & 169 & $0.09[0.05,0.16]$ & $0.86[0.81,0.91]$ & $=$ \\
\hline Vang $2020 \mathrm{~b}$ & 13 & 3 & 296 & 157 & $0.04[0.02,0.07]$ & $0.98[0.95,1.00]$ & $\frac{1}{1} 1 \frac{1}{1} 1$ \\
\hline
\end{tabular}

\section{Test 27. Urea increase}

Urea increase

Study TP FP FN TN Sensitivity $[95 \% \mathrm{Cl})$ Specificity $(95 \% \mathrm{Cl})$ Sensitivity (95\% Cl)Specificity (95\% Cl) $\begin{array}{llllllll}\text { Chen } 2020 \mathrm{c} & 1 & 9 & 77 & 17 & 0.01[0.00,0.07] & 0.65[0.44,0.83]\end{array}$ Yang 2020b $\quad \begin{array}{lllllll}6 & 3 & 299 & 157 & 0.02[0.01,0.04] & 0.98[0.95,1.00]\end{array}$

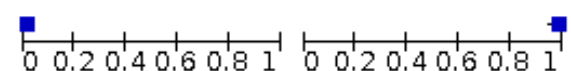




\title{
Test 28. ALT increase
}

\begin{abstract}
ALT increase
\end{abstract}

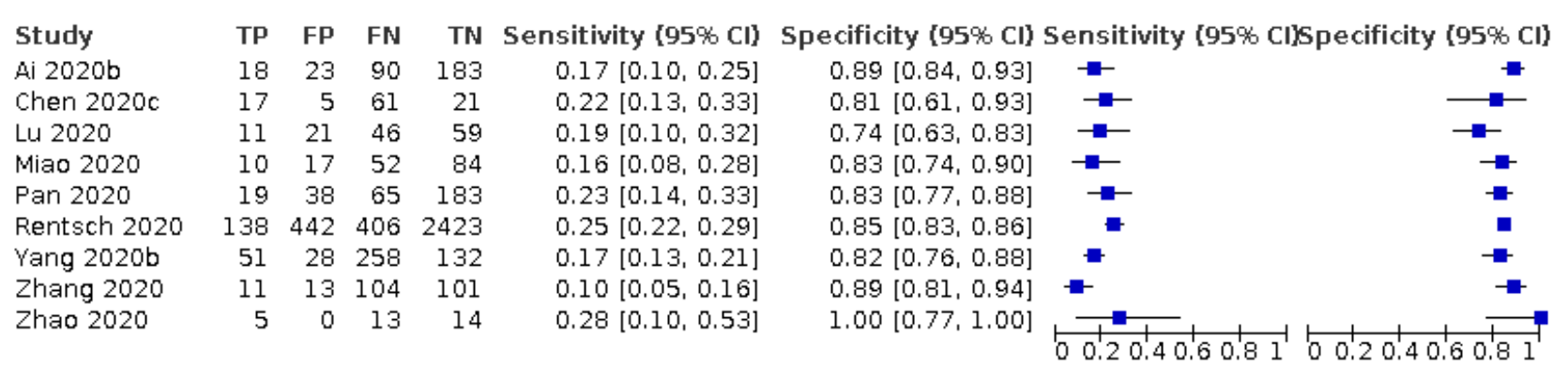

Test 29. AST increase

AST increase

$\begin{array}{lrrrrr}\text { Study } & \text { TP } & \text { FP } & \text { FN } & \text { TN } & \text { Sensitivity }[95 \% \mathrm{Cl}) \\ \text { Ai 2020b } & 28 & 46 & 80 & 165 & 0.26[0.18,0.35] \\ \text { Chen } 2020 \mathrm{c} & 18 & 5 & 60 & 21 & 0.23[0.14,0.34] \\ \text { Pan 2020 } & 32 & 43 & 52 & 178 & 0.38[0.28,0.49] \\ \text { Rentsch 2020 } & 157 & 374 & 391 & 2511 & 0.29[0.25,0.33] \\ \text { Yang 2020b } & 50 & 17 & 259 & 143 & 0.16[0.12,0.21] \\ \text { Zhang 2020 } & 17 & 25 & 98 & 89 & 0.15[0.09,0.23] \\ \text { Zhao 2020 } & 5 & 0 & 13 & 14 & 0.28[0.10,0.53]\end{array}$

Specificity (95\% Cl) Sensitivity (95\% Cl)Specificity (95\% Cl) $0.78[0.72,0.84]$ $0.81[0.61,0.93]$ $0.81[0.75,0.86]$ $0.87[0.86,0.88]$ $0.89[0.84,0.94]$ $0.78[0.69,0.85]$ $1.00[0.77,1.00]$

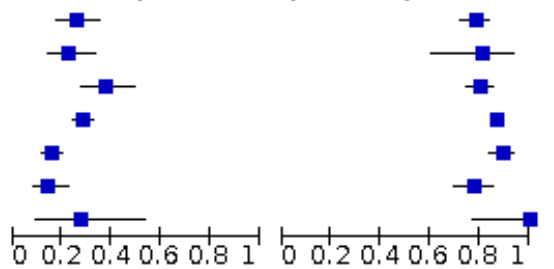

\section{Test 30. Total bilirubin (TBIL) increase}

Total bilirubin (TBIL) increase

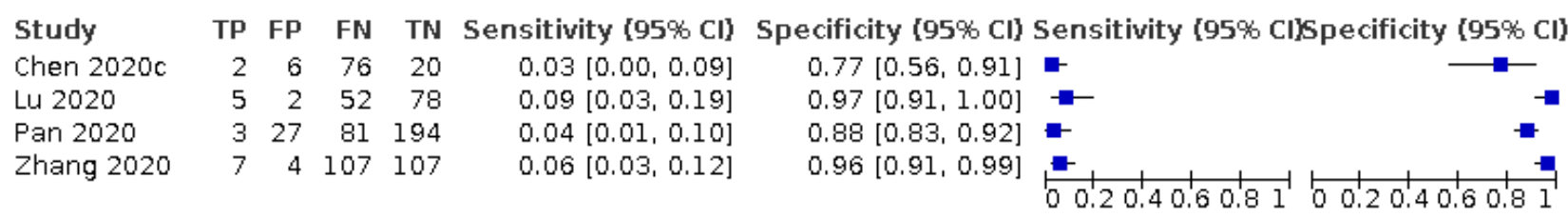

\section{Test 31. Erythrocyte Sedimentation Rate (ESR) increase}

Erythrocyte Sedimentation Rate (ESR) increase

Study TP FP FN TN Sensitivity $(95 \% \mathrm{Cl})$ Specificity $(95 \% \mathrm{Cl}$ ) Sensitivity (95\% Cl)Specificity $[95 \% \mathrm{Cl}$ ) $\begin{array}{lllllll}\text { Ai } 2020 b & 61 & 83 & 38 & 97 & 0.62[0.51,0.71] & 0.54[0.46,0.61]\end{array}$

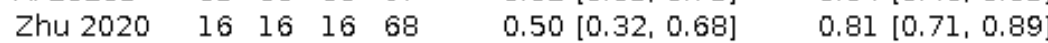

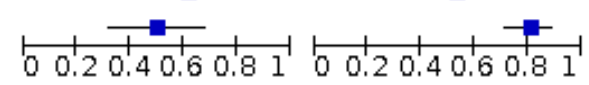


Test 32. CRP increase

CRP increase

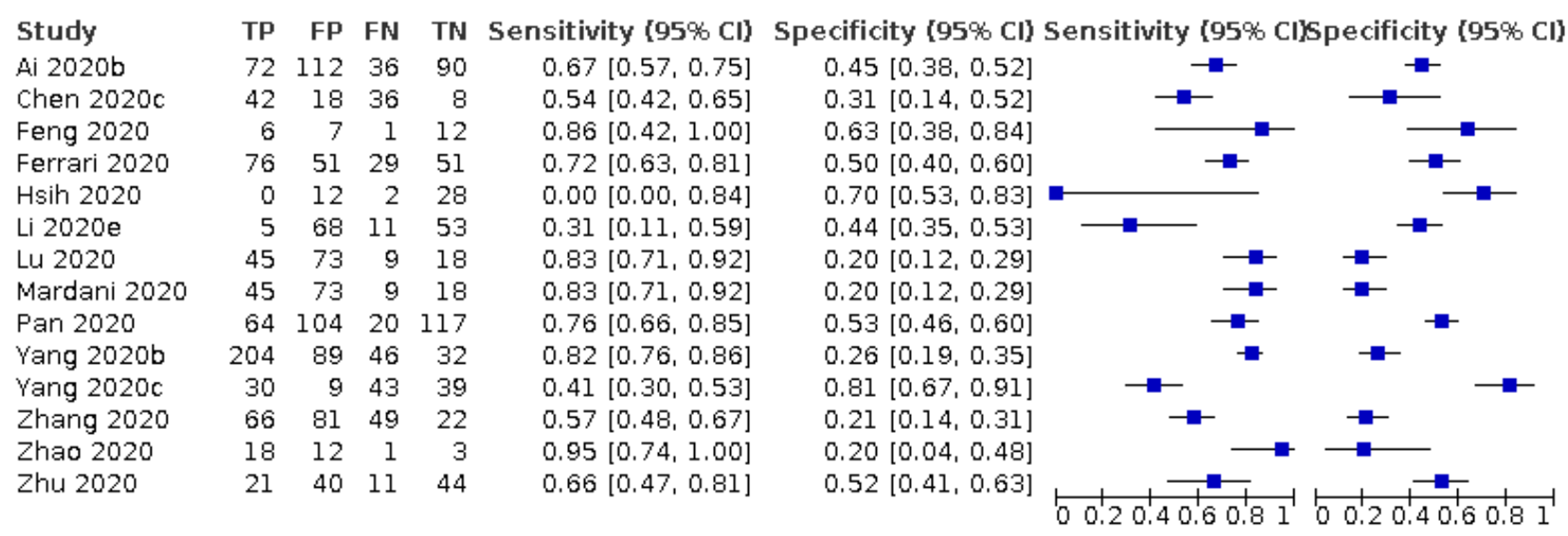

\section{Test 33. a-HBDH increased}

a-HBDH increased

Study TP FP FN TN Sensitivity $(95 \% \mathrm{Cl})$ Specificity $(95 \% \mathrm{Cl})$ Sensitivity $(95 \% \mathrm{Cl})$ Specificity (95\% Cl) $\begin{array}{llllllll}\text { Ai } 2020 \mathrm{~b} & 37 & 61 & 71 & 135 & 0.34[0.25,0.44] & 0.69[0.62,0.75\end{array}$ Zhao $2020 \quad 6 \quad \begin{array}{llllll}6 & 2 & 12 & 0.75[0.35,0.97] & 0.80[0.52,0.96]\end{array}$

Test 34. HCT increased

HCT increased

Study TP FP FN TN Sensitivity $(95 \% \mathrm{Cl})$ Specificity $(95 \% \mathrm{Cl})$ Sensitivity $(95 \% \mathrm{Cl})$ Specificity (95\% Cl)

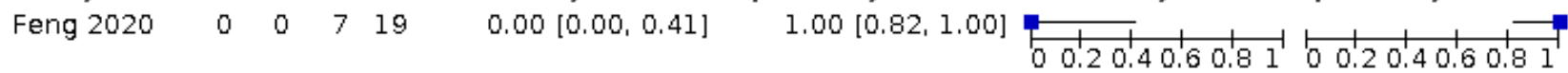

\section{Test 35. HCT decreased}

\section{HCT decreased}

Study TP FP FN TN Sensitivity $(95 \% \mathrm{Cl})$ Specificity $(95 \% \mathrm{Cl})$ Sensitivity $(95 \% \mathrm{Cl})$ Specificity (95\% Cl) $\begin{array}{lrrrrrrr}\text { Feng } 2020 & 1 & 3 & 6 & 16 & 0.14[0.00,0.58] & 0.84[0.60,0.97] \rightarrow- & \\ \text { Pan } 2020 & 38 & 190 & 46 & 31 & 0.45[0.34,0.56] & 0.14[0.10,0.19] & -\end{array}$ 
Test 36. Albumin (ALB) decreased

Albumin (ALB) decreased

\begin{tabular}{|c|c|c|c|c|c|c|c|c|}
\hline Study & TP & FP & FN & TN & Sensitivity $(95 \% \mathrm{Cl})$ & Specificity $(95 \% \mathrm{Cl})$ & Sensitivity $\left(95^{\circ}\right.$ & $\%$ Cl)Specificity $(95 \% \mathrm{Cl})$ \\
\hline Lu 2020 & 31 & 34 & 25 & 42 & $0.55[0.41,0.69]$ & $0.55[0.43,0.67]$ & $\rightarrow-$ & $\rightarrow-$ \\
\hline Pan 2020 & 34 & 185 & 50 & 36 & $0.40[0.30,0.52]$ & $0.16[0.12,0.22]$ & $\rightarrow-$ & 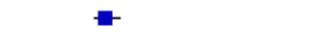 \\
\hline Rentsch 2020 & 129 & 681 & 415 & 2181 & $0.24[0.20,0.28]$ & $0.76[0.75,0.78]$ & $=$ & - \\
\hline Zhang 2020 & 5 & 15 & 110 & 99 & $0.04[0.01,0.10]$ & $0.87[0.79,0.92]$ & 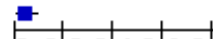 & \\
\hline
\end{tabular}

Test 37. Globulin (GLB) increase

Globulin (GLB) increase

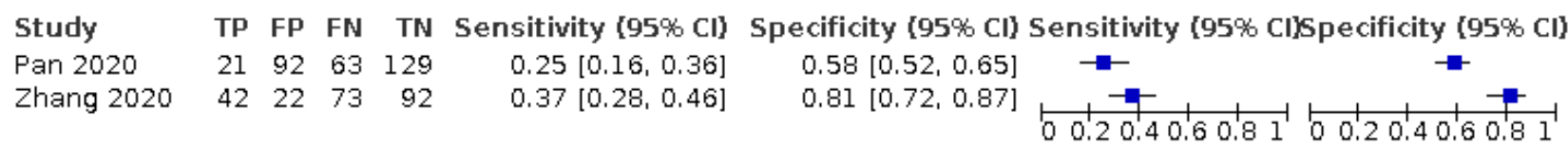

\section{Test 38. Globulin (GLB) decrease}

Globulin (GLB) decrease

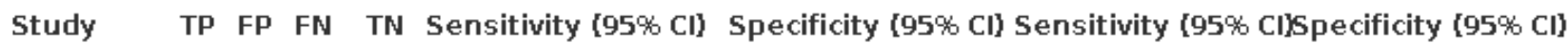
$\begin{array}{lllllll}\text { Pan } 2020 & 1 & 20 & 83 & 201 & 0.01 & {[0.00,0.06}\end{array} \quad 0.91[0.86,0.94]$

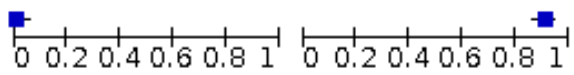

\section{Test 39. Procalcitonin (PCT) increase}

Procalcitonin $(\mathrm{PCT})$ increase

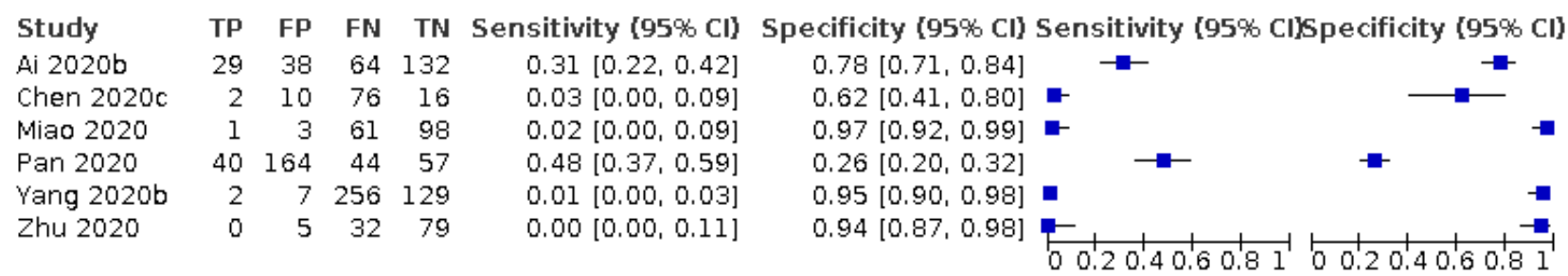

Test 40. eGFR

eGFR

Study TP FP FN TN Sensitivity $(95 \% \mathrm{Cl})$ Specificity $(95 \% \mathrm{Cl})$ Sensitivity $[95 \% \mathrm{Cl}] \mathrm{Specificity}(95 \% \mathrm{Cl})$ Rentsch $2020 \quad 27 \quad 1025412951 \quad 0.05[0.03,0.07] \quad 0.97[0.96,0.97]$

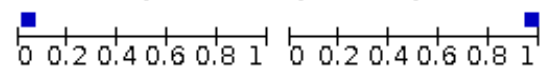


Test 41. Proteinuria

\section{Proteinuria}

Study TP FP FN TN Sensitivity $[95 \% \mathrm{Cl}$ ) Specificity $[95 \% \mathrm{Cl}$ ) Sensitivity $[95 \% \mathrm{Cl}$ )Specificity $[95 \% \mathrm{Cl}$ ) Liu $2020 \quad 34 \quad 5 \quad 85 \quad 40 \quad 0.29[0.21,0.38] \quad 0.89[0.76,0.96]$

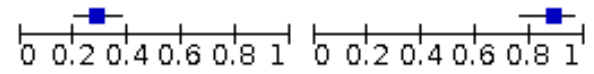

Test 42. Prothrombin time (PT) increase

Prothrombin time $(\mathrm{PT})$ increase

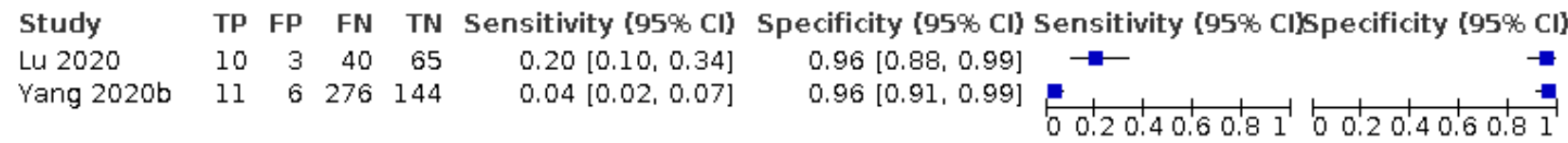

Test 43. GGT increased

GGT increased

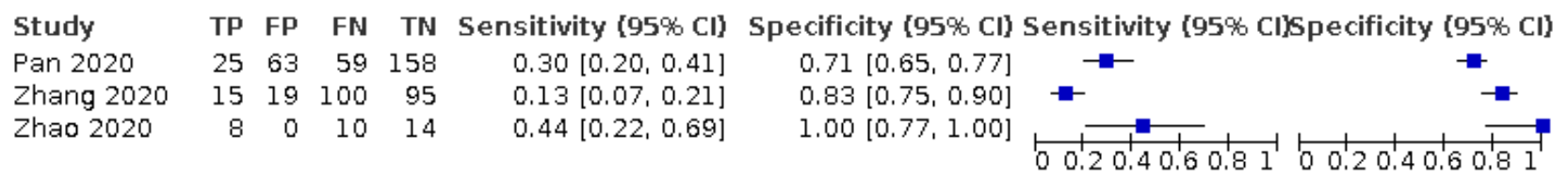

Test 44. D-dimer increase

D-dimer increase

Study TP FP FN TN Sensitivity $(95 \% \mathrm{Cl})$ Specificity $[95 \% \mathrm{Cl}$ ) Sensitivity $[95 \%$ Cl $)$ Specificity $(95 \% \mathrm{Cl})$ $\begin{array}{lllrrrr}\text { Lu } 2020 & 21 & 25 & 24 & 36 & 0.47[0.32,0.62] & 0.59[0.46,0.71] \\ \text { Yang 2020b } & 59 & 36 & 228 & 114 & 0.21[0.16,0.26] & 0.76[0.68,0.83]\end{array}$ $\begin{array}{lrrrrrrr}\text { Yang 2020b } & 59 & 36 & 228 & 114 & 0.21[0.16,0.26] & 0.76[0.68,0.83] \\ \text { Zhu 2020 } & 3 & 9 & 29 & 75 & 0.09[0.02,0.25] & 0.89[0.81,0.95]\end{array}$

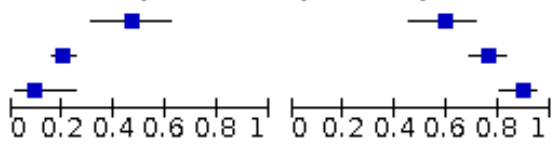

Test 45. IL-2

IL-2

Study TP FP FN TN Sensitivity $(95 \% \mathrm{Cl})$ Specificity $(95 \% \mathrm{Cl}$ ) Sensitivity $[95 \%$ Cl]Specificity $[95 \% \mathrm{Cl}$ ) Li $2020 \mathrm{~d} \quad 30 \quad 5 \quad 10 \quad 11 \quad 0.75[0.59,0.87] \quad 0.69[0.41,0.89]$

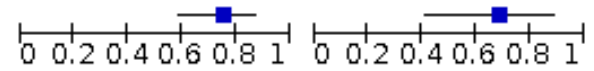

Test 46. IL-4

IL-4

Study TP FP FN TN Sensitivity $[95 \% \mathrm{Cl}$ ) Specificity $(95 \% \mathrm{CI}$ ) Sensitivity $[95 \%$ Cl)Specificity (95\% Cl) Li $2020 \mathrm{~d} \quad 30 \quad 5 \quad 10 \quad 11 \quad 0.75[0.59,0.87] \quad 0.69[0.41,0.89]$

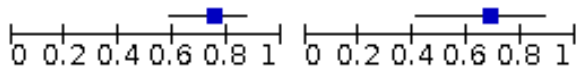


Test 47. Interleukin-6 (IL-6) increase

Interleukin-6 (IL-6) increase

$\begin{array}{lrrrrrr}\text { Study } & \text { TP } & \text { FP } & \text { FN } & \text { TN } & \text { Sensitivity }(95 \% \mathrm{CI}) & \text { Specificity }(95 \% \mathrm{CI}) \text { Sensitivity }(95 \% \mathrm{Cl} \text { Specificity (95\% CI) } \\ \text { Feng 2020 } & 6 & 10 & 1 & 9 & 0.86[0.42,1.00] & 0.47[0.24,0.71] \\ \text { Li 2020d } & 30 & 5 & 10 & 11 & 0.75[0.59,0.87] & 0.69[0.41,0.89] \\ \text { Zhao 2020 } & 6 & 8 & 1 & 3 & 0.86[0.42,1.00] & 0.27[0.06,0.61] \\ \text { Zhu 2020 } & 7 & 7 & 25 & 77 & 0.22[0.09,0.40] & 0.92[0.84,0.97]\end{array}$

Test 48. IL-8

IL-8

Study TP FP FN TN Sensitivity $(95 \% \mathrm{Cl})$ Specificity $(95 \% \mathrm{Cl}$ ) Sensitivity (95\% Cl)Specificity (95\% Cl) Li 2020d $30 \quad 5 \quad 10 \quad 11 \quad 0.75[0.59,0.87] \quad 0.69[0.41,0.89]$

$\frac{1}{0} \frac{1}{1+20.40 .60 .81} \frac{1}{0.1} \frac{1}{0.20 .40 .60 .81}$

Test 49. IL-10

IL-10

Study TP FP FN TN Sensitivity $[95 \% \mathrm{Cl}$ ) Specificity $(95 \% \mathrm{CI}$ ) Sensitivity $[95 \%$ Cl)Specificity (95\% Cl) Li 2020d $30 \quad 5 \quad 10 \quad 11 \quad 0.75[0.59,0.87] \quad 0.69[0.41,0.89]$

$\frac{1}{0} \frac{1}{1+20.40 .60 .81} \frac{1}{0.1} \frac{1}{0.20 .40 .60 .81}$

\section{Test 50. TNF alpha}

TNF alpha

Study TP FP FN TN Sensitivity $(95 \% \mathrm{Cl})$ Specificity $(95 \% \mathrm{CI}$ ) Sensitivity (95\% Cl)Specificity (95\% Cl) Li $2020 \mathrm{~d} \quad 30 \quad 5 \quad 10 \quad 11 \quad 0.75[0.59,0.87] \quad 0.69[0.41,0.89]$

$\frac{1}{0.20 .40 .60 .81}+\frac{1}{0.20 .40 .60 .81}$

Test 51. ALP increased

ALP increased

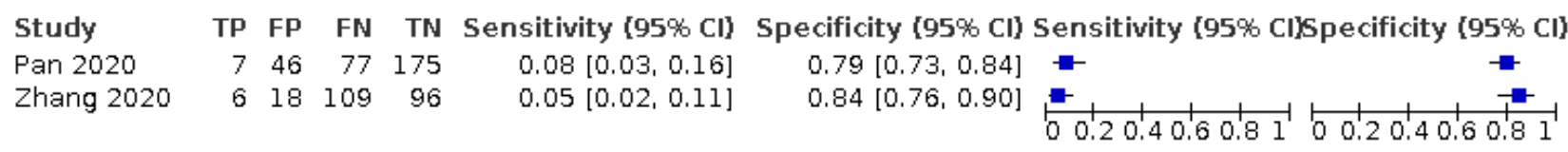


Test 52. pro-BNP

pro-BNP

Study TP FP FN TN Sensitivity $(95 \% \mathrm{Cl})$ Specificity $(95 \% \mathrm{Cl}$ ) Sensitivity $(95 \% \mathrm{Cl}$ Specificity (95\% Cl)

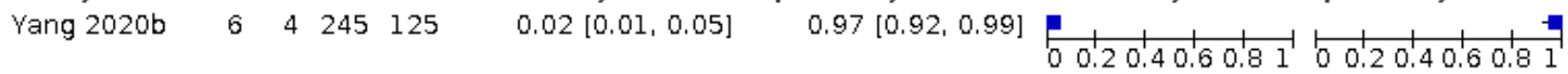

\section{Test 53. Hematuria}

Hematuria

Study TP FP FN TN Sensitivity $(95 \% \mathrm{Cl})$ Specificity $(95 \% \mathrm{Cl})$ Sensitivity $(95 \%$ Cl)Specificity $(95 \% \mathrm{Cl})$ Liu $2020 \quad 49 \quad 10 \quad 70 \quad 35 \quad 0.41[0.32,0.51] \quad 0.78[0.63,0.89]$

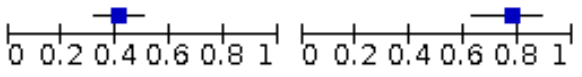

Test 54. INR increase

INR increase

$\begin{array}{lrrrrrr}\text { Study } & \text { TP } & \text { FP } & \text { FN } & \text { TN } & \text { Sensitivity }(95 \% \mathrm{Cl}) & \text { Specificity }(95 \% \mathrm{CI}) \text { Sensitivity }(95 \% \mathrm{Cl} \text { Specificity (95\% Cl) } \\ \text { Yang 2020b } & 20 & 14 & 267 & 136 & 0.07[0.04,0.11] & 0.91[0.85,0.95] \\ \text { Zhang 2020 } & 60 & 32 & 55 & 74 & 0.52[0.43,0.62] & 0.70[0.60,0.78]\end{array}$

Test 55. LDH increase

LDH increase

$\begin{array}{lrrrrrrr}\text { Study } & \text { TP } & \text { FP } & \text { FN } & \text { TN } & \text { Sensitivity }(95 \% \mathrm{Cl}) & \text { Specificity }(95 \% \mathrm{Cl}) \text { Sensitivity (95\% Cl]Specificity (95\% Cl) } \\ \text { Ai 2020b } & 32 & 54 & 76 & 142 & 0.30[0.21,0.39] & 0.72[0.66,0.79] \\ \text { Chen 2020c } & 11 & 8 & 67 & 18 & 0.14[0.07,0.24] & 0.69[0.48,0.86] \\ \text { Miao 2020 } & 17 & 39 & 45 & 62 & 0.27[0.17,0.40] & 0.61[0.51,0.71] \\ \text { Zhang 2020 } & 26 & 21 & 89 & 72 & 0.23[0.15,0.31] & 0.77[0.68,0.85] \\ \text { Zhao 2020 } & 6 & 0 & 13 & 15 & 0.32[0.13,0.57] & 1.00[0.78,1.00]\end{array}$

Test 56. Mean corpuscular volume increase

Mean corpuscular volume increase

Study TP FP FN TN Sensitivity $[95 \% \mathrm{Cl})$ Specificity $(95 \% \mathrm{Cl})$ Sensitivity (95\% Cl)Specificity (95\% Cl)

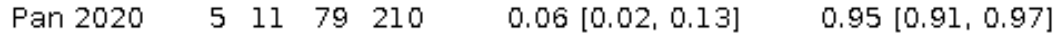

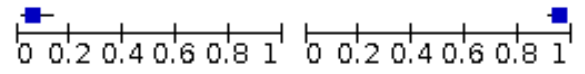

Test 57. Mean corpuscular volume decrease

Mean corpuscular volume decrease

Study TP FP FN TN Sensitivity $(95 \% \mathrm{Cl})$ Specificity $(95 \% \mathrm{Cl})$ Sensitivity (95\% Cl)Specificity (95\% Cl) Pan $2020 \quad 38 \quad 190 \quad 46 \quad 31 \quad 0.45[0.34,0.56] \quad 0.14[0.10,0.19]$

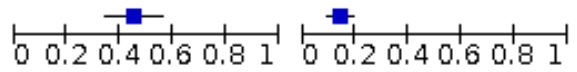


Test 58. Erythrocyte mean corpuscular hemoglobin increase

Erythrocyte mean corpuscular hemoglobin increase

Study TP FP FN TN Sensitivity $(95 \% \mathrm{Cl})$ Specificity $(95 \% \mathrm{Cl})$ Sensitivity $(95 \%$ Cl $)$ Specificity (95\% Cl)

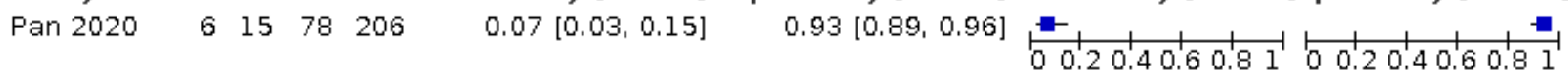

Test 59. Erythrocyte mean corpuscular hemoglobin decrease

Erythrocyte mean corpuscular hemoglobin decrease

Study TP FP FN TN Sensitivity $(95 \% \mathrm{Cl})$ Specificity $(95 \% \mathrm{Cl})$ Sensitivity (95\% Cl)Specificity (95\% Cl) $\left.\begin{array}{lllllll}\text { Pan } 2020 \quad 4 \quad 22 & 80 & 199 & 0.05 & {[0.01,0.12]} & 0.90 & {[0.85,0.94}\end{array}\right]$

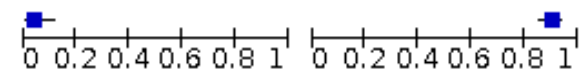

Test 60. Erythrocytemean corpuscular hemoglobin concentrate increase

Erythrocytemean corpuscular hemoglobin concentrate increase

Study TP FP FN TN Sensitivity $(95 \% \mathrm{Cl})$ Specificity $(95 \% \mathrm{Cl})$ Sensitivity (95\% Cl)Specificity (95\% Cl)

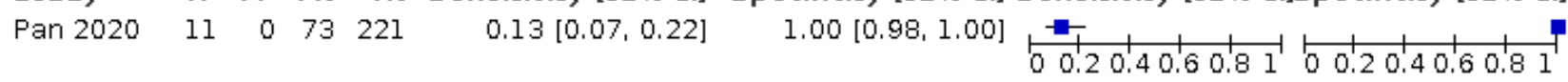

Test 61. Erythrocytemean corpuscular hemoglobin concentrate decrease

Erythrocytemean corpuscular hemoglobin concentrate decrease

Study TP FP FN TN Sensitivity $[95 \% \mathrm{Cl})$ Specificity $(95 \% \mathrm{Cl})$ Sensitivity $(95 \%$ Cl $)$ Specificity (95\% Cl) $\left.\begin{array}{lllllll}\text { Pan } 2020 & 0 & 24 & 84 & 197 & 0.00 & {[0.00,0.04}\end{array}\right] \quad 0.89[0.84,0.93]$

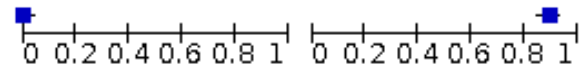

Test 62. Mean Platelet Volume

Mean Platelet Volume

Study TP FP FN TN Sensitivity $(95 \% \mathrm{Cl})$ Specificity $(95 \% \mathrm{Cl})$ Sensitivity $(95 \% \mathrm{Cl})$ Specificity (95\% Cl) $\begin{array}{lllllll}\text { Pan } 2020 & 3 & 4 & 81 & 217 & 0.04 & {[0.01,0.10}\end{array} \quad 0.98[0.95,1.00]$

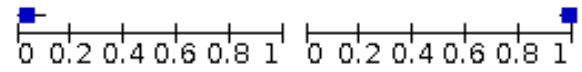

\section{Test 63. Direct bilirubin}

Direct bilirubin

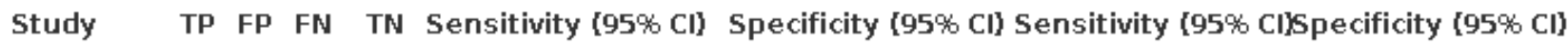
Pan $2020 \quad 12 \quad 3372188 \quad 0.14[0.08,0.24] \quad 0.85[0.80,0.89]$ 
Test 64. unconjugated bilirubin

unconjugated bilirubin

Study TP FP FN TN Sensitivity $[95 \% \mathrm{Cl}$ ) Specificity $(95 \% \mathrm{Cl}$ ) Sensitivity $(95 \% \mathrm{Cl}$ )Specificity $[95 \% \mathrm{Cl})$ $\begin{array}{lllllll}\text { Pan } 2020 \quad 2 & 23 & 82 & 198 & 0.02 & {[0.00,0.08}\end{array} \quad 0.90[0.85,0.93]$

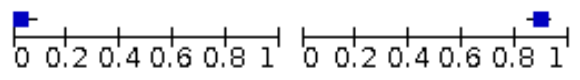

Test 65. Total protein

Total protein

Study TP FP FN TN Sensitivity $(95 \% \mathrm{Cl})$ Specificity $(95 \% \mathrm{Cl})$ Sensitivity $(95 \% \mathrm{Cl})$ Specificity $(95 \% \mathrm{Cl})$ Pan $2020 \quad 19 \quad 115 \quad 65106 \quad 0.23[0.14,0.33] \quad 0.48[0.41,0.55]$

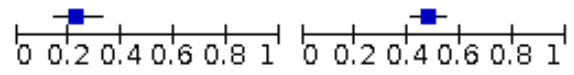

Test 66. Total bile acid

Total bile acid

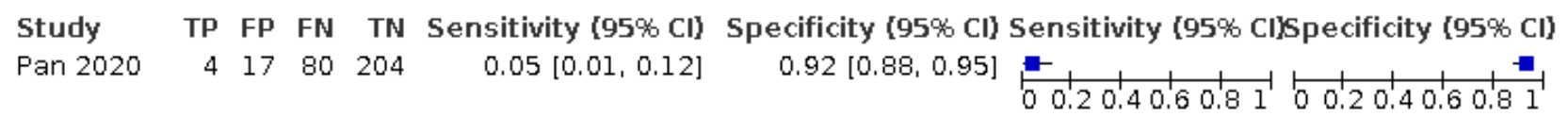

Test 67. Troponin I

Troponin I

Study TP FP FN TN Sensitivity $(95 \% \mathrm{Cl})$ Specificity $(95 \% \mathrm{Cl})$ Sensitivity $(95 \% \mathrm{Cl})$ Specificity (95\% Cl)

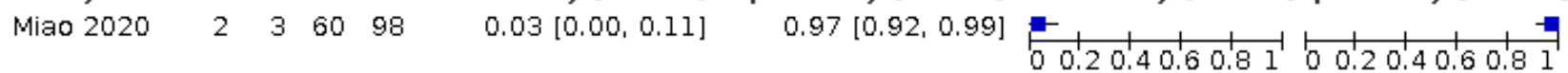

ADDITIONALTABLES

Table 1. QUADAS-2 checklist

\begin{tabular}{|c|c|c|c|c|c|}
\hline Index test(s) & $\begin{array}{l}\text { Review \#1. Labora- } \\
\text { tory based molecu- } \\
\text { lar tests }\end{array}$ & $\begin{array}{l}\text { Review \#2. Point- } \\
\text { of-care tests }\end{array}$ & $\begin{array}{l}\text { Review \#3. Anti- } \\
\text { body tests }\end{array}$ & $\begin{array}{l}\text { Review \#4. Signs } \\
\text { and symptoms }\end{array}$ & $\begin{array}{l}\text { Review \#5. Routine } \\
\text { laboratory tests }\end{array}$ \\
\hline $\begin{array}{l}\text { Patients } \\
\text { (setting, } \\
\text { intended } \\
\text { use of index } \\
\text { test, presen- } \\
\text { tation, prior } \\
\text { testing) }\end{array}$ & $\begin{array}{l}\text { Considered to be the } \\
\text { 'gold standard' for } \\
\text { acute infection. } \\
\text { May have been used } \\
\text { with different sam- } \\
\text { ples, in different set- } \\
\text { tings, for case-find- } \\
\text { ing or confirmation } \\
\text { of infection in pa- } \\
\text { tients with suspect- } \\
\text { ed COVID-19. }\end{array}$ & $\begin{array}{l}\text { In patients with sus- } \\
\text { pected COVID-19 or } \\
\text { contact tracing. } \\
\text { Point-of-care: case- } \\
\text { finding in the gener- } \\
\text { al population, care } \\
\text { homes for elderly } \\
\text { people, emergency } \\
\text { departments. }\end{array}$ & $\begin{array}{l}\text { In patients with } \\
\text { signs and symp- } \\
\text { toms suspected of } \\
\text { COVID-19 and for } \\
\text { case finding; also in } \\
\text { patients with past } \\
\text { exposure to SARS- } \\
\text { CoV-2. }\end{array}$ & $\begin{array}{l}\text { General practice, } \\
\text { primary care, emer- } \\
\text { gency care. } \\
\text { In patients present- } \\
\text { ing with suspected } \\
\text { COVID-19. } \\
\text { No prior testing. } \\
\text { Signs and symptoms } \\
\text { often used for triage } \\
\text { or referral. }\end{array}$ & $\begin{array}{l}\text { Mainly meant for sit- } \\
\text { uations where a lab- } \\
\text { oratory was close; } \\
\text { emergency care, } \\
\text { hospital, ICU. COVID } \\
\text { triage centres. } \\
\text { In patients present- } \\
\text { ing with suspected } \\
\text { COVID-19. }\end{array}$ \\
\hline
\end{tabular}


Table 1. QUADAS-2 checklist (Continued)

Reference The focus will be on the diagnosis of COVID-19 pneumonia or infection with SARS-CoV-2. For this protocol, the focus standard will not be on prognosis.

and target

condition

\section{PARTICIPANT SELECTION}

Was a con- This will be similar for all index tests, target conditions, and populations.

\section{secutive}

or random

sample of

patients en-

rolled?
YES: if a study explicitly stated that all participants within a certain time frame were included; that this was done consecutively; or that a random selection was done.

NO: if it was clear that a different selection procedure was employed; for example, selection based on clinician's preference, or based on institutions.

UNCLEAR: if the selection procedure was not clear or not reported.

Was a case- This will be similar for all index tests, target conditions, and populations.

control de-

sign avoid-

ed?

YES: if a study explicitly stated that all participants came from the same group of (suspected) patients.

NO: if it was clear that a different selection procedure was employed for the participants depending on their COVID-19 (pneumonia) status or SARS-CoV-2 infection status.

UNCLEAR: if the selection procedure was not clear or not reported.

\section{Did the} study avoid inappropriate exclusions?

Studies may have excluded patients, or selected patients in such a way that they avoided including those who were difficult to diagnosis or likely to be borderline. Although the inclusion and exclusion criteria will be different for the different index tests, inappropriate exclusions and inclusions will be similar for all index tests: for example, only elderly patients excluded, or children (as sampling may be more difficult). This needs to be addressed on a case-tocase basis.

YES: if a high proportion of eligible patients was included without clear selection.

NO: if a high proportion of eligible patients was excluded without providing a reason; if, in a retrospective study, participants without index test or reference standard results were excluded; if exclusion was based on severity assessment postfactum or comorbidities (cardiovascular disease, diabetes, immunosuppression).

UNCLEAR: if the exclusion criteria were not reported.

\section{Did the} study avoid inappropriate inclusions?
Some laboratory studies may have intentionally included groups of patients in whom the accuracy was likely to differ, such as those with particularly low or high viral loads, or who had other diseases, such that the sample over-represented these groups. This needs to be addressed on a case-to-case basis. Artificial spiked samples are a clear example.

YES: if samples included were likely to be representative of the spectrum of disease.

NO: if the study oversampled patients with particular characteristics likely to affect estimates of accuracy.

UNCLEAR: if the exclusion criteria were not reported.
Could the selection of patients have intro- duced bias? lead to bias.
LOW: if all signalling questions were answered with YES.
UNCLEAR: all other instances.

HIGH: if one or more signalling questions were answered with NO, as any deviation from the selection process may

\begin{tabular}{llllll}
$\begin{array}{l}\text { Is there con- } \\
\text { cern that }\end{array}$ & HIGH: if accuracy of & HIGH: if accuracy of & HIGH: if accuracy of & HIGH: if accuracy & HIGH: if accuracy of \\
the included & in a case-control de- & tests was assessed & tests was assessed & of signs and symp- & laboratory tests was \\
patients do & sign; to screen con- & sign; if not used to & $\begin{array}{l}\text { in a case-control } \\
\text { design; when pa- }\end{array}$ & $\begin{array}{l}\text { toms were assessed } \\
\text { in a case-control }\end{array}$ & $\begin{array}{l}\text { assessed in a case- } \\
\text { control design, or in }\end{array}$ \\
\hline
\end{tabular}


Table 1. QUADAS-2 checklist (Continued) not match the review question?

\section{tacts or for stopping} contact isolation. Studies done in sample banks and spiked samples.

LOW: any other situation: these tests may be used in different settings and for different purposes.

UNCLEAR: if a description about the participants was lacking.

\section{diagnose early acute tients were test-} infection; to screen contacts or for stopping contact isolation. Studies done in sample banks and spiked samples.

LOW: any other situation: these tests may have been used in different settings and for different purposes.

UNCLEAR: if a description about the participants was lacking. ed too early in the disease phase for detection of past infection. Studies done in sample banks and spiked samples.

LOW: any other situation: these tests may be used in different settings and for different purposes.

UNCLEAR: if a description about the participants was lacking. design, or in an already highly selected group of participants, or the study was able to only estimate sensitivity or specificity.

LOW: any situation where signs and symptoms were the first assessment/test to be done on the included participants.

UNCLEAR: if a de-

scription about the participants was lacking. an already highly selected group of participants.

LOW: any situation where generic laboratory tests were among the first tests to be done on the included participants.

UNCLEAR: if a description about the participants was lacking.

\section{INDEX TESTS}

\begin{tabular}{|c|c|}
\hline Were the in- & This will be similar for all index tests, target cc \\
\hline $\begin{array}{l}\text { sults inter- } \\
\text { preted with- }\end{array}$ & $\begin{array}{l}\text { YES: if blinding was explicitly stated or index } t \\
\text { available. }\end{array}$ \\
\hline $\begin{array}{l}\text { out knowl- } \\
\text { edge of the } \\
\text { results of }\end{array}$ & $\begin{array}{l}\text { NO: if it was explicitly stated that the index te } \\
\text { ence standard. }\end{array}$ \\
\hline $\begin{array}{l}\text { the refer- } \\
\text { ence stan- } \\
\text { dard? }\end{array}$ & UNCLEAR: if blinding was unclearly re \\
\hline
\end{tabular}

If a thresh- This will be similar for all index tests, target conditions, and populations.

old was used, was it prespecified? 
Table 1. QUADAS-2 checklist (Continued) the review question? nel not available in practice. a different setting, or using samples, equipment, or personnel not available in practice.

\section{REFERENCE STANDARD}

$\begin{array}{ll}\begin{array}{l}\text { Is the refer- } \\ \text { ence stan- }\end{array} & \begin{array}{l}\text { In this review, we focused on the target condition COVID-19 disease. Although we defined acceptable reference stan- } \\ \text { dard likely }\end{array} \\ \begin{array}{l}\text { the eligible studies, Studies of which it is clear that only RT-PCR was used will be considered high risk of bias. } \\ \text { to correctly }\end{array} & \\ \text { classify the } & \\ \text { target con- } & \\ \text { dition? } & \end{array}$

Were the reference standard results interpreted without knowledge of the results of the index test?
YES: if it was explicitly stated that the reference standard results were interpreted without knowledge of the results of the index test, or if the result of the index test was obtained after the reference standard.

NO: if it was explicitly stated that the reference standard results were interpreted with knowledge of the results of the index test or if the index test was used to make the final diagnosis.

UNCLEAR: if blinding was unclearly reported.

\section{Did the defi- nition of the reference standard in- corporate results from the index test(s)?}

YES: if results from the index test were a component of the reference standard definition.

NO: if the reference standard did not incorporate the index standard test.

UNCLEAR: if it was unclear whether the results of the index test formed part of the reference standard.

\section{Could the conduct or interpreta- tion of the reference standard have intro- duced bias?}

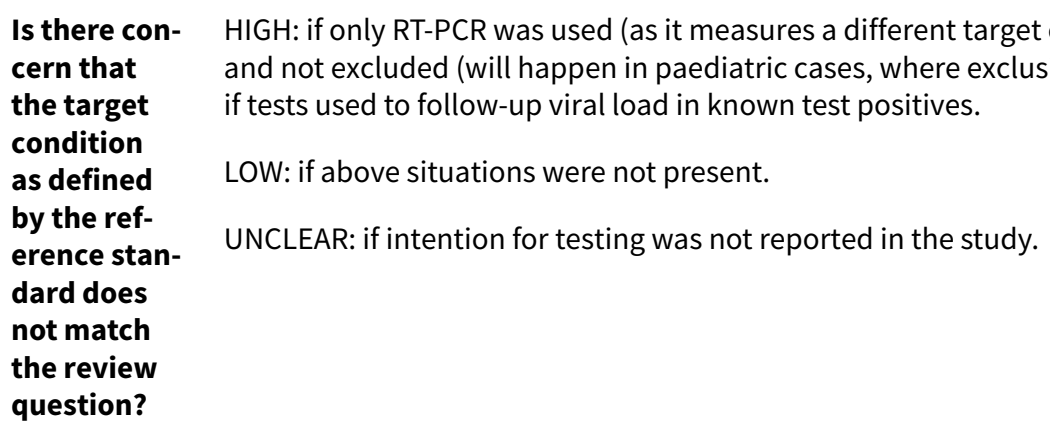

\section{FLOW AND TIMING}


Table 1. QUADAS-2 checklist (Continued)

\begin{abstract}
Was there YES: this will be similar for all index tests, populations for the current infection target conditions: as the situation of a an appropri- patient, including clinical presentation and disease progress, evolves rapidly and new/ongoing exposure can result ate interval in case status change, an appropriate time interval will be within 24 hours. For testing for previous infection, a time between in- interval of at least two weeks is required since resolution of symptoms before the index test was undertaken.
\end{abstract}

dex test(s)

and refer-

NO: if there was more than 24 hours between the index test and the reference standard or if patients were otherwise

ence stan-

dard? reported to be assessed with the index versus reference standard test at moments of different severity.

UNCLEAR: if the time interval was not reported.

Did all pa- $\quad$ YES: if all patients received a reference standard (clearly no partial verification).

tients re-

ceive a ref- NO: if only (part of) the index test positives or index test negatives received the complete reference standard.

erence stan-

dard?

UNCLEAR: if it was not reported.

Did all pa- $\quad$ YES: if all patients received the same reference standard (clearly no differential verification).

tients re-

ceive the

same refer-

ence stan-

dard?

NO: if (part of) the index test positives or index test negatives received a different reference standard.

UNCLEAR: if it was not reported.

Were all pa-

tients in-

cluded in

the analy-

sis?

YES: if all included patients were included in the analyses as well.

NO: if after the inclusion/exclusion process, patients were removed from the analyses for different reasons: no reference standard done, no index test done, intermediate results of both index test or reference standard, indeterminate results of both index test or reference standard, samples unusable.

UNCLEAR: if this was not clear from the reported numbers.
Could the
HIGH: if one or more signalling questions were answered with NO.
patient flow
have intro-
LOW: if all signalling questions were answered with YES.
duced bias?
UNCLEAR: all other instances.

ICU: intensive care unit; RT-PCR: reverse transcriptase polymerase chain reaction; SARS-CoV-2: severe acute respiratory syndrome coronavirus 2; WHO: World Health Organization 


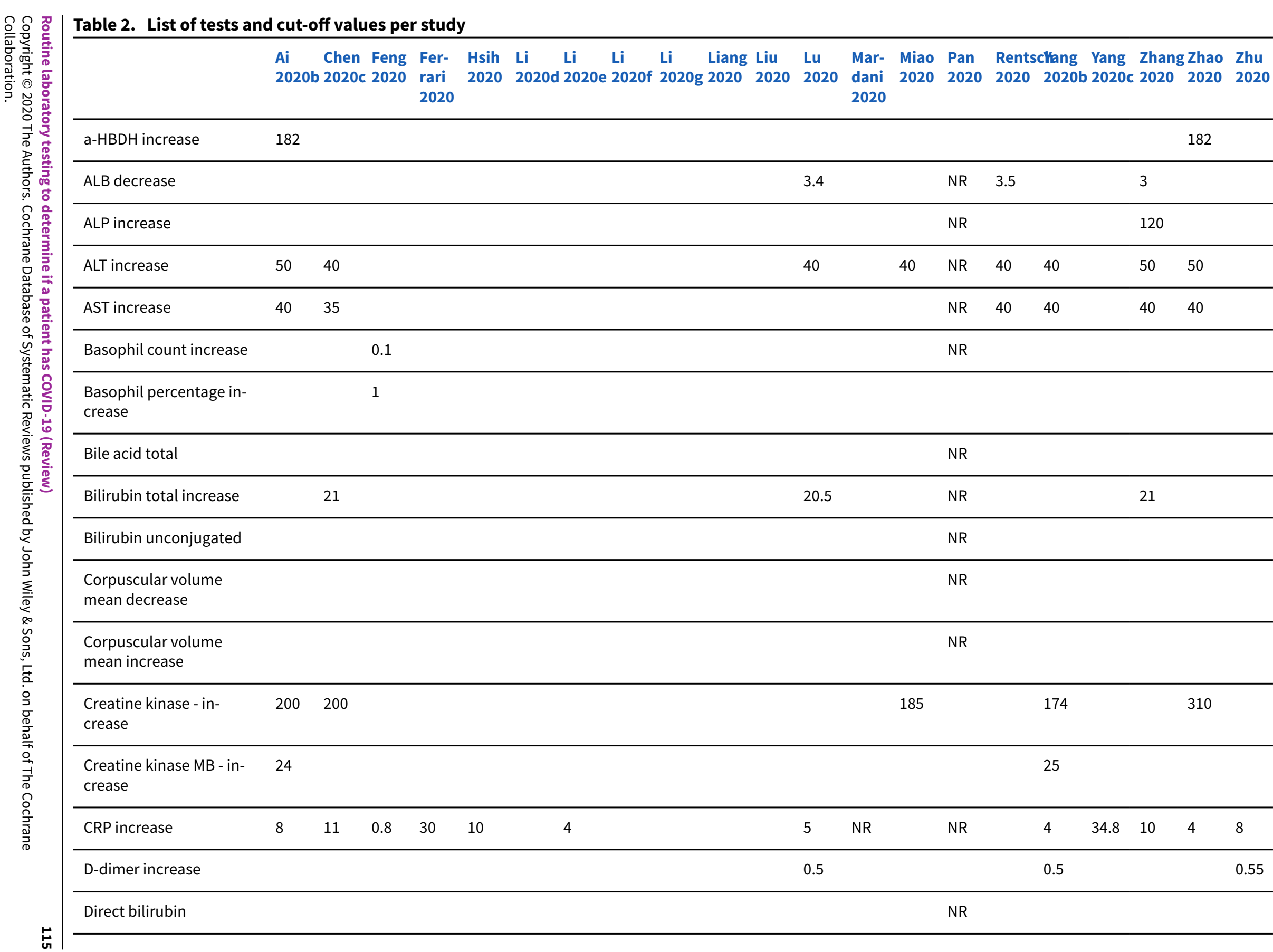




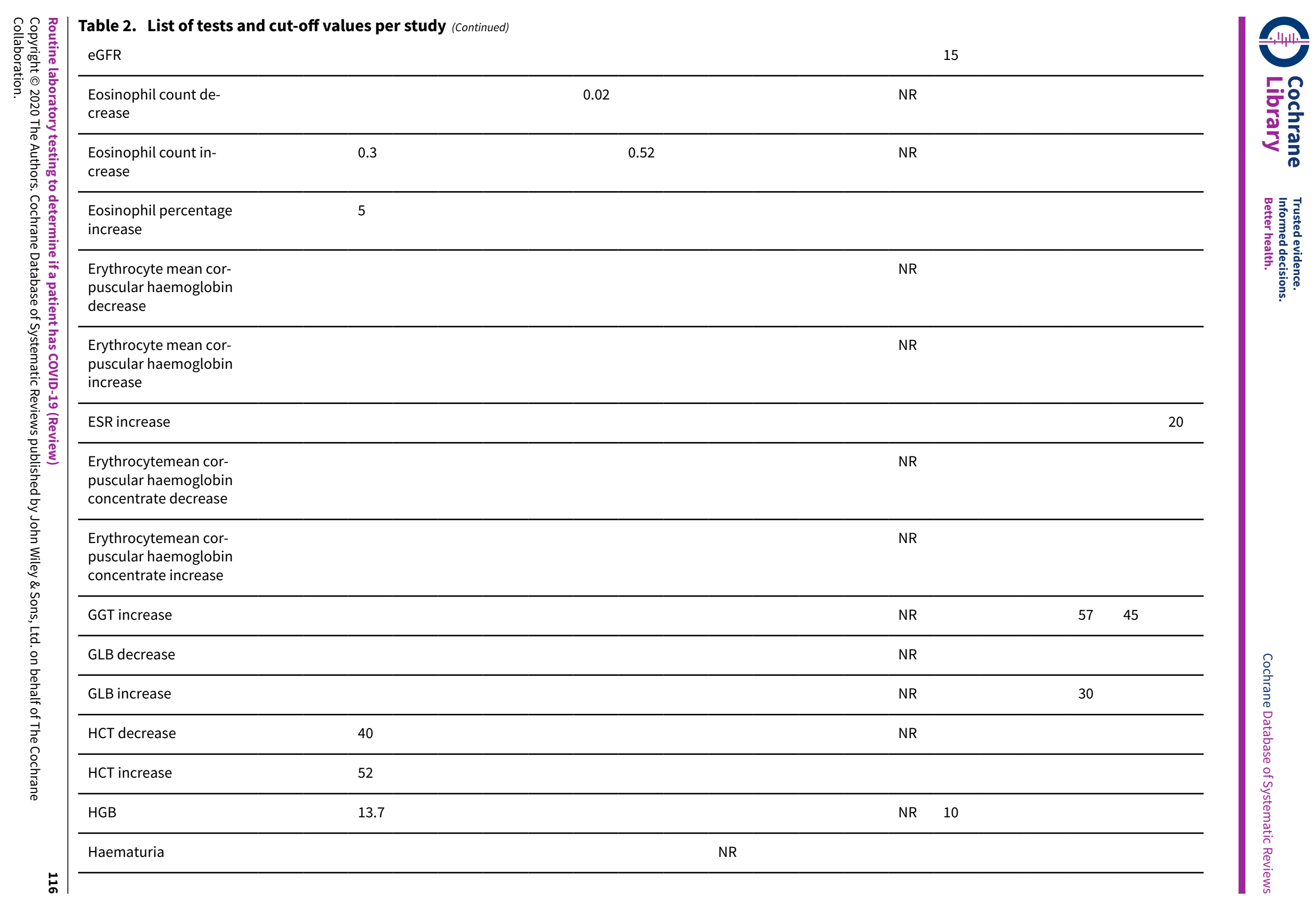




\begin{tabular}{|c|c|c|c|c|c|c|c|c|c|c|c|c|c|c|c|c|c|}
\hline & IL-10 & & & & & NR & & & & & & & & & & & \\
\hline 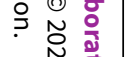 & IL-2 & & & & & NR & & & & & & & & & & & \\
\hline न-1 & $\mathrm{IL}-4$ & & & & & NR & & & & & & & & & & & \\
\hline 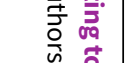 & IL-6 increase & & & 5.9 & & NR & & & & & & & & & & 7 & 7 \\
\hline 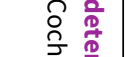 & IL-8 & & & & & NR & & & & & & & & & & & \\
\hline 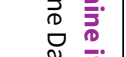 & INR increase & & & & & & & & & & & & 1.25 & & 1.15 & & \\
\hline 幽 & LDH increase & 250 & 250 & & & & & & & & 245 & & & & 243 & 250 & \\
\hline$\underbrace{\vec{n}}_{n} \vec{F}$ & Leukocyturia & & & & & & & & & NR & & & & & & & \\
\hline 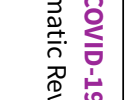 & $\begin{array}{l}\text { Lymphocyte count de- } \\
\text { crease }\end{array}$ & 1.1 & 1.1 & 1 & 1 & & 1.1 & 1.1 & 1.1 & 1.1 & NR & 0.8 & 0.8 & & & 1.1 & 1.1 \\
\hline 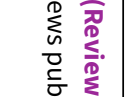 & $\begin{array}{l}\text { Lymphocyte count in- } \\
\text { crease }\end{array}$ & 3.2 & & 4 & & & & & & & NR & & & & & & \\
\hline $\begin{array}{l}\overrightarrow{0} \\
\stackrel{0}{a} \\
\underset{\Sigma}{0}\end{array}$ & $\begin{array}{l}\text { Lymphocyte percentage } \\
\text { decrease }\end{array}$ & & 20 & 20 & & & & & & & & & & 23.7 & & & 20 \\
\hline$\sum_{\substack{\bar{D} \\
ٍ}}^{5}$ & $\begin{array}{l}\text { Lymphocyte percentage } \\
\text { increase }\end{array}$ & & & 40 & & & & & & & & & & & & & \\
\hline 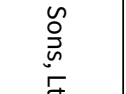 & $\begin{array}{l}\text { Monocyte count de- } \\
\text { crease }\end{array}$ & 0.1 & & & & & & & & & NR & & & & & & \\
\hline $\begin{array}{l}\frac{0}{\partial} \\
\frac{0}{0} \\
\frac{0}{2}\end{array}$ & $\begin{array}{l}\text { Monocyte count in- } \\
\text { crease }\end{array}$ & 0.6 & & 0.8 & & & & 0.6 & & & NR & & & & & & \\
\hline 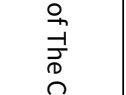 & $\begin{array}{l}\text { Monocyte percentage in- } \\
\text { crease }\end{array}$ & & & 8 & & & & & & & & & & & & & \\
\hline 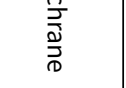 & $\begin{array}{l}\text { Neutrophil count de- } \\
\text { crease }\end{array}$ & 1.8 & & 2 & & & & & 1.8 & & NR & & & & & & \\
\hline & $\begin{array}{l}\text { Neutrophil count in- } \\
\text { crease }\end{array}$ & 6.3 & 6.3 & 7 & & & 6.3 & 6.3 & 6.3 & 6.3 & NR & & 7 & 4.61 & & & 6.3 \\
\hline
\end{tabular}




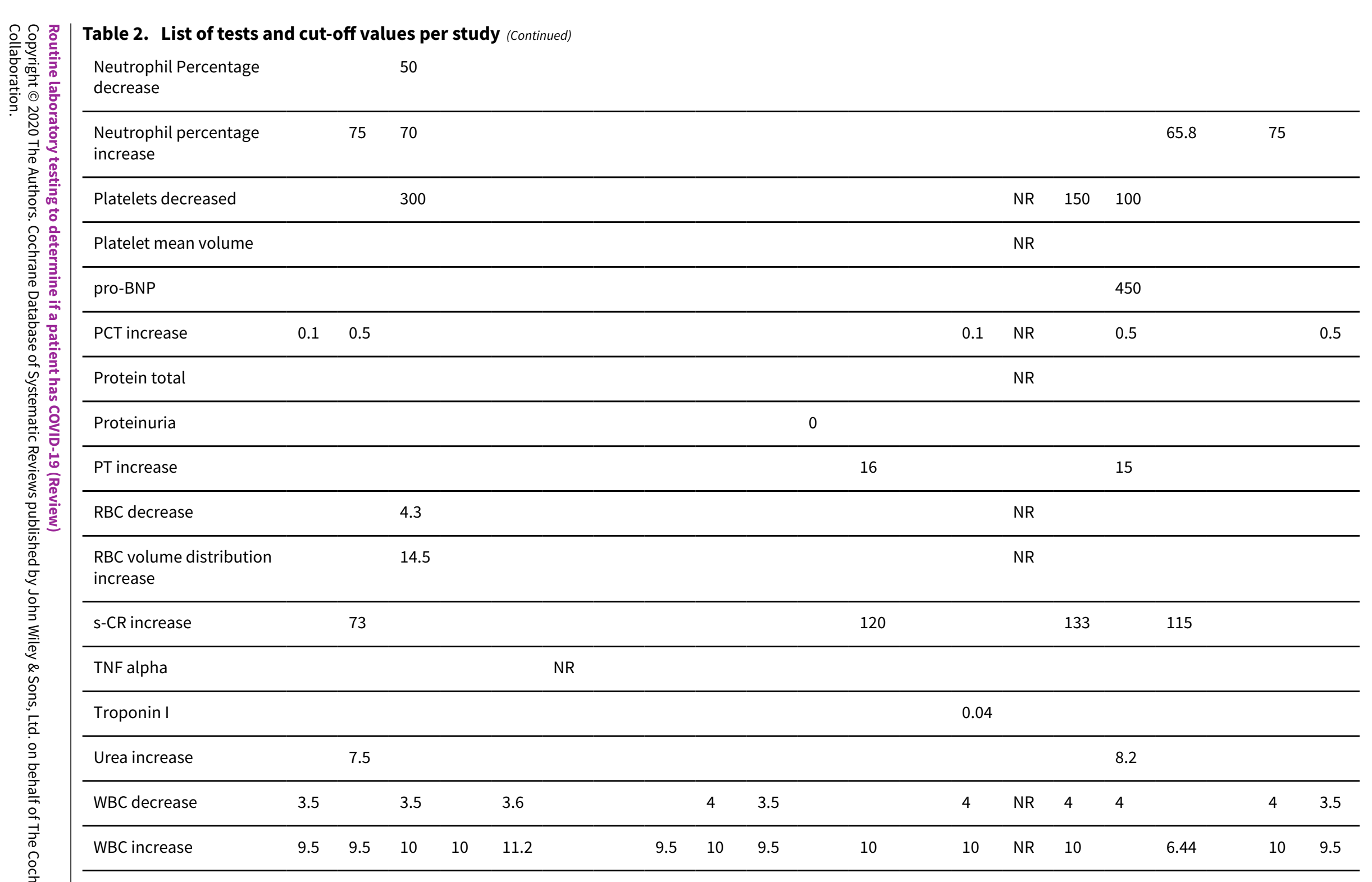

a-HBDH: $\alpha$-Hydroxybutyrate dehydrogenase; ALB: albumin; ALP: alkaline phosphatase; ALT: alanine aminotransferase; AST: aspartate aminotransferase; CRP: C-reactive protein; eGFR: estimated glomerular filtration rate; ESR: erythrocyte sedimentation rate; GGT: gamma-glutamyl transferase; GLB: globulin; HCT: haematocrit; HGB: haemoglobin; IL: interleukin; INR: international normalized ratio; LDH: lactate dehydrogenase; pro-BNP: pro B-type natriuretic peptide; PCT: procalcitonin; PT: prothrombin time; RBC: red blood cell; s-CR: serum creatinine; TNF: tumour necrosis factor; WBC: white blood cell 

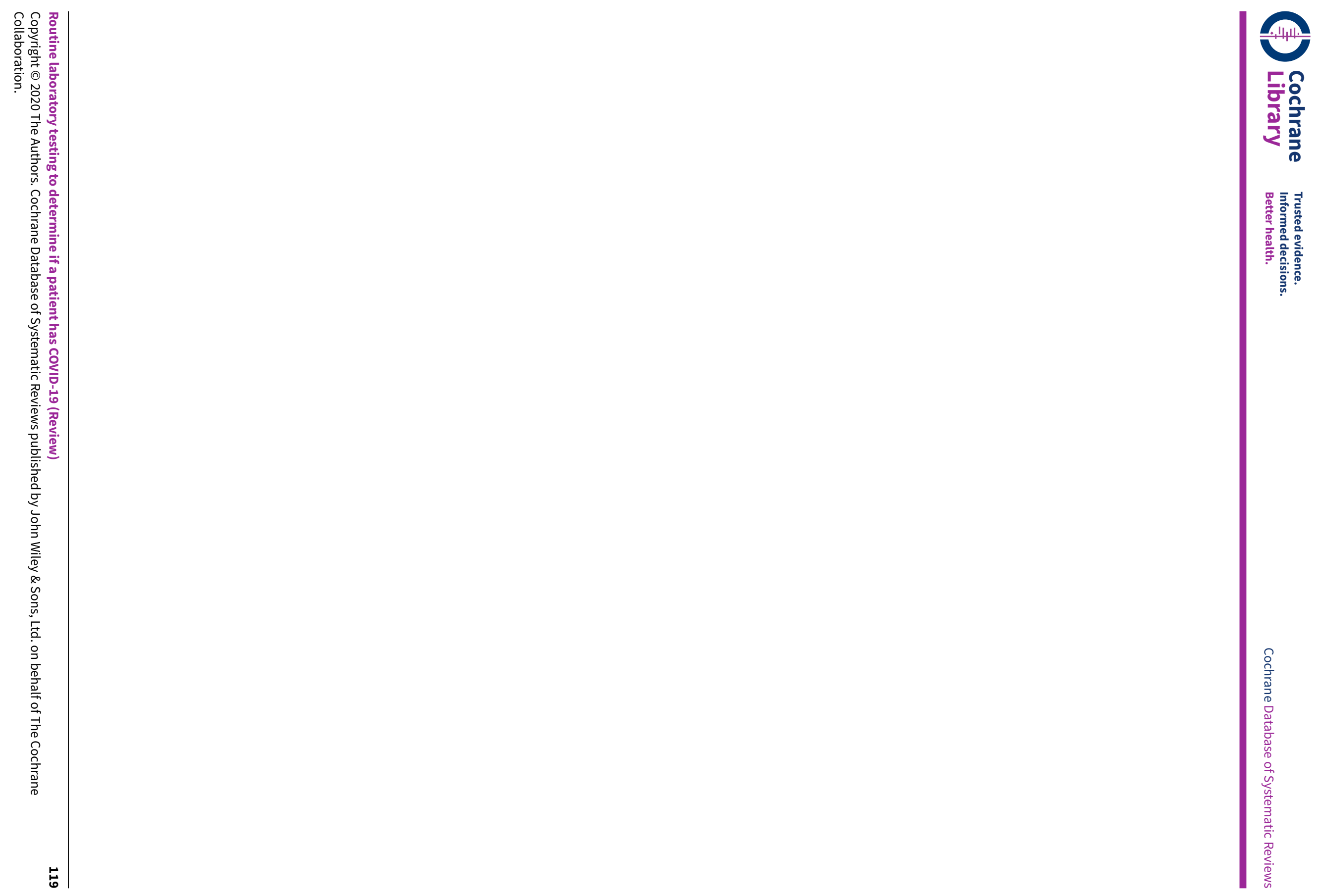


\section{A P PE N D I CES}

\section{Appendix 1. World Health Organization case definitions}

\section{Severe pneumonia}

Adolescent or adult: fever or suspected respiratory infection, plus one of the following: respiratory rate > 30 breaths/minute; severe respiratory distress; or oxygen saturation $\left(\mathrm{SpO}_{2}\right) \leq 93 \%$ on room air. Child with cough or difficulty in breathing, plus at least one of the following: central cyanosis or $\mathrm{SpO}_{2}<90 \%$; severe respiratory distress (for example, grunting, very severe chest indrawing); signs of pneumonia with a general danger sign: inability to breastfeed or drink, lethargy or unconsciousness, or convulsions.

Other signs of pneumonia may be present: chest indrawing, fast breathing (in breaths/minute): aged < 2 months: $\geq 60$; aged 2 to 11 months: $\geq 50$; aged 1 to 5 years: $\geq 40$. While the diagnosis is made on clinical grounds; chest imaging may identify or exclude some pulmonary complications.

\section{Acute respiratory distress syndrome (ARDS)}

Onset within one week of a known clinical insult or new or worsening respiratory symptoms.

Chest imaging (that is, X-ray, computer tomography scan, or lung ultrasound): bilateral opacities, not fully explained by volume overload, lobar or lung collapse, or nodules.

Origin of pulmonary infiltrates: respiratory failure not fully explained by cardiac failure or fluid overload. Need objective assessment (for example, echocardiography) to exclude hydrostatic cause of infiltrates/oedema if no risk factor present.

Oxygenation impairment in adults:

- mild ARDS: $200 \mathrm{mmHg}$ < ratio of arterial oxygen partial pressure/fractional inspired oxygen $\left(\mathrm{PaO}_{2} / \mathrm{FiO}_{2}\right) \leq 300 \mathrm{mmHg}$ (with positive endexpiratory pressure (PEEP) or continuous positive airway pressure (CPAP) $\geq 5 \mathrm{cmH}_{2} \mathrm{O}$, or non-ventilated);

- moderate ARDS: $100 \mathrm{mmHg}<\mathrm{PaO}_{2} / \mathrm{FiO}_{2} \leq 200 \mathrm{mmHg}$ (with PEEP $\geq 5 \mathrm{cmH}_{2} \mathrm{O}$, or non-ventilated);

- severe ARDS: $\mathrm{PaO}_{2} / \mathrm{FiO}_{2} \leq 100 \mathrm{mmHg}$ (with PEEP $\geq 5 \mathrm{cmH}_{2} \mathrm{O}$, or non-ventilated);

- when $\mathrm{PaO}_{2}$ is not available, $\mathrm{SpO}_{2} / \mathrm{FiO}_{2} \leq 315 \mathrm{mmHg}$ suggests ARDS (including in non-ventilated patients).

Oxygenation impairment in children: note $\mathrm{OI}=$ Oxygenation Index and OSI = Oxygenation Index using $\mathrm{SpO}_{2}$. Use $\mathrm{PaO}_{2}$-based metric when available. If $\mathrm{PaO}_{2}$ not available, wean $\mathrm{FiO}_{2}$ to maintain $\mathrm{SpO}_{2} \leq 97 \%$ to calculate $\mathrm{OSI}$ or $\mathrm{SpO}_{2} / \mathrm{FiO}_{2}$ ratio:

- bilevel (non-invasive ventilation or CPAP) $\geq 5 \mathrm{cmH}_{2} \mathrm{O}$ via full-face mask: $\mathrm{PaO}_{2} / \mathrm{FiO}_{2} \leq 300 \mathrm{mmHg}$ or $\mathrm{SpO}_{2} / \mathrm{FiO}_{2} \leq 264$;

- mild ARDS (invasively ventilated): $4 \leq \mathrm{OI}<8$ or $5 \leq \mathrm{OSI}<7.5$;

- moderate ARDS (invasively ventilated): $8 \leq \mathrm{OI}<16$ or $7.5 \leq \mathrm{OSI}<12.3$;

- severe ARDS (invasively ventilated): $\mathrm{OI} \geq 16$ or $\mathrm{OSI} \geq 12.3$.

\section{Appendix 2. Cochrane COVID-19 Study Register searches}

\begin{tabular}{ll}
\hline Source & Strategy \\
\hline CT.gov & COVID-19a \\
\hline WHO ICTRP & Health topic: 2019-nCov/COVID-19 \\
\hline PubMed & $($ "2019 nCoV"[tiab] OR 2019nCoV[tiab] OR "2019 novel coronavirus"[tiab] OR "COVID 19"[tiab] \\
OR COVID19[tiab] OR "new coronavirus"[tiab] OR "novel coronavirus"[tiab] OR "novel coro- \\
na virus"[tiab] OR "SARS CoV-2"[tiab] OR (Wuhan[tiab] AND (coronavirus[tiab] OR "corona \\
virus"[tiab])) OR "COVID-19"[Supplementary Concept] OR "severe acute respiratory syndrome \\
coronavirus 2"[Supplementary Concept]) NOT ("animals"[MeSH Terms] NOT "humans"[MeSH \\
Terms])) NOT (editorial[pt] OR comment[pt] OR letter[pt] OR newspaper article[pt])
\end{tabular}


aAutomatic term mapping links results for 2019-nCoV, 2019 novel coronavirus, SARS-CoV-2, severe acute respiratory syndrome coronavirus 2.

\section{Ovid Embase Search}

Embase records from the Stephen B. Thacker CDC Library, Covid-19 Research articles Downloadable database. Records were obtained by the CDC library by searching Embase through Ovid using the following search strategy:

(coronavir* OR corona virus* OR betacoronavir* OR covid19 OR covid 19 OR nCoV OR novel CoV OR CoV 2 OR CoV2 OR sarscov2 OR 2019nCoV OR wuhan virus $)$.mp. OR ((wuhan OR hubei OR huanan) AND (severe acute respiratory OR pneumonia*) AND outbreak $\left.{ }^{\star}\right)$.mp. OR Coronavirus infection/ OR coronavirinae/ OR exp betacoronavirus/

Limits: 2020-

OR

(novel coronavir* OR novel corona virus* OR covid19 OR covid 19 OR nCoV OR novel CoV OR CoV 2 OR CoV2 OR sarscov2 OR 2019nCoV OR wuhan virus $)^{\star}$.mp. OR ((wuhan OR hubei OR huanan) AND (severe acute respiratory OR pneumonia*) AND outbreak*).mp. OR ((wuhan OR hubei OR huanan) AND (coronavir* OR betacoronavir*)).mp.

Limits: 2019-

\section{Appendix 3. Living search from the University of Bern}

The following information is taken from the university of Bern website (see: ispmbern.github.io/covid-19/living-review/ collectingdata.html).

The register is updated daily and CSV file downloads are made available.

\section{April 2020}

From 1 April 2020, we will retrieve the curated bioRxiv/medRxiv dataset (connect.medrxiv.org/relate/content/181).

\section{6 to 31 March 2020}

MEDLINE: (\"Wuhan coronavirus\" [Supplementary Concept] OR \"COVID-19\" OR \"2019 ncov\"[tiab] OR ((\"novel coronavirus\"[tiab] OR ।"new coronavirus\"[tiab]) AND (wuhan[tiab] OR 2019[tiab])) OR 2019-nCoV[All Fields] OR (wuhan[tiab] AND coronavirus[tiab])))))

Embase: (nCoV or 2019-nCoV or ((new or novel or wuhan) adj3 coronavirus) or covid19 or covid-19 or SARS-CoV-2).mp.

bioRxiv/medRxiv: ncov or corona or wuhan or COVID or SARS-CoV-2

With the kind support of the Public Health \& Primary Care Library PHC (www.unibe.ch/university/services/university_library/ faculty_libraries/medicine/public_health_amp_primary_care_library_phc/index_eng.html), and following guidance of the Medical Library Association (www. mlanet.org/p/cm/ld/fid=1713).

\section{January 2020 to 25 March 2020}

MEDLINE: ("Wuhan coronavirus" [Supplementary Concept] OR "COVID-19" OR "2019 ncov"[tiab] OR (("novel coronavirus"[tiab] OR "new coronavirus"[tiab]) AND (wuhan[tiab] OR 2019[tiab])) OR 2019-nCoV[All Fields] OR (wuhan[tiab] AND coronavirus[tiab])))))

Embase: ncov OR (wuhan AND corona) OR COVID

bioRxiv/medRxiv: ncov or corona or wuhan or COVID

\section{H I S T O R Y}

Review first published: Issue 11, 2020

\section{CONTRIBUTIONSOF AUTHORS}

Inge Stegeman: Study selection, data-extraction and quality assessment, first draft of the review and subsequent revisions; Eleanor A Ochodo: Study selection, data-extraction and quality assessment, multiple revisions of the review;

Fatuma Guleid: Study selection, data-extraction and quality assessment, multiple revisions of the review;

Gea A. Holtman: Study selection, data-extraction and quality assessment, multiple revisions of the review; 
Bada Yang: Study selection, data-extraction and quality assessment, multiple revisions of the review;

Jane Cunningham contributed clinical, methodological and/or technical expertise to drafting the protocol; contributed to multiple revisions of the review;

Clare Davenport contributed clinical, methodological and/or technical expertise to drafting the protocol; contributed to multiple revisions of the review;

Jonathan J Deeks: contributed clinical, methodological and/or technical expertise to drafting the protocol; contributed to multiple revisions of the review and co-ordinated all contributions to all Cochrane Rapid DTA reviews;

Jacqueline Dinnes contributed clinical, methodological and/or technical expertise to drafting the protocol; did the initial screening titles and abstracts for all reviews; contributed to multiple revisions of the review;

Sabine Dittrich contributed clinical, methodological and/or technical expertise to drafting the protocol; contributed to multiple revisions of the review;

Devy Emperador contributed clinical, methodological and/or technical expertise to drafting the protocol; contributed to multiple revisions of the review;

Lotty Hooft contributed clinical, methodological and/or technical expertise to drafting the protocol; contributed to multiple revisions of the review;

René Spijker contributed clinical, methodological and/or technical expertise to drafting the protocol; co-ordinated and conducted the study retrieval en initial selection steps; contributed to multiple revisions of the review;

Yemisi Takwoingi contributed clinical, methodological and/or technical expertise to drafting the protocol; supervised the meta-analyses; contributed to multiple revisions of the review;

Ann Van den Bruel contributed clinical, methodological and/or technical expertise to drafting the protocol; contributed to multiple revisions of the review;

Junfeng Wang translated articles from Chinese to English whenever necessary; retrieved articles in Chinese; extracted data from and assessed quality of Chinese language articles; contributed to revised versions of the review;

Miranda Langendam: Study selection, data-extraction and quality assessment, multiple revisions of the review;

Jan Verbakel: Study selection, data-extraction and quality assessment, meta-analyses; multiple revisions of the review;

Mariska MG Leeflang contributed clinical, methodological and/or technical expertise to drafting the protocol; drafted the QUADAS-2 criteria; co-ordinated the review process; overall supervision; drafted all non-automatic Tables; GRADE assessment; contributed to the first draft and subsequent revisions of the review.

\section{DECLARATIONS OF INTEREST}

Inge Stegeman: has provided freelance consultancy for approved professional organizations and learned societies (physiotherapists, optometrists, opticians), and has no known conflicts of interest in relation to this review.

Eleanor A Ochodo: none known

Fatuma Guleid: none known.

Gea A. Holtman: none known.

Bada Yang: none known.

Jane Cunningham: none known.

Clare Davenport: none known.

Jonathan J Deeks: none known.

Jacqueline Dinnes: none known.

Sabine Dittrich: is employed by FIND. FIND has several clinical research projects to evaluate multiple new diagnostic tests against published Target Product Profiles that have been defined through consensus processes. These studies are for diagnostic products developed by 
private sector companies who provide access to know-how, equipment/reagents, and contribute through unrestricted donations as per FIND policy and external SAC review.

Devy Emperador: is employed by FIND. FIND has several clinical research projects to evaluate multiple new diagnostic tests against published Target Product Profiles that have been defined through consensus processes. These studies are for diagnostic products developed by private sector companies who provide access to know-how, equipment/reagents, and contribute through unrestricted donations as per FIND policy and external SAC review.

Lotty Hooft: none known.

René Spijker: the Dutch Cochrane Centre (DCC) has received grants for performing commissioned systematic reviews. In no situation, the commissioner had any influence on the results of the work.

Yemisi Takwoingi: none known.

Ann Van den Bruel: none known.

Junfeng Wang: has received consultancy fee from Biomind, an Artificial Intelligence (AI) company providing machine intelligence solutions in medical imaging. The consultancy service was about design of clinical studies, not related to this review. The company had no influence on the results of the work.

Miranda Langendam: none known.

Jan Verbakel: none known.

Mariska MG Leeflang: none known.

\section{SOURCES OF SUPPORT}

\section{Internal sources}

- Liverpool School of Tropical Medicine, UK

\section{External sources}

- Foreign, Commonwealth and Development Office (FCDO), UK

Project number: 300342-104

- National Institute for Health Research (NIHR), UK

\section{DIFFERENCES BETWEEN PROTOCOL AND REVIEW}

We deviated from our protocol on some occasions. We intended to include studies that recruited only COVID-19 cases, to estimate sensitivity or those restricted to people without COVID-19, to estimate specificity (Deeks 2020a). We decided to deviate from this rule as the added value of such studies for our review is questionable.

We planned to investigate test accuracy, either by stratified analysis or meta-regression, according to a specific measurement or biomarker, days of symptoms, severity of symptoms, reference standard, sample type, study design, and setting. We decided not to do these analyses in the first version of this review because of the lack of primary studies per subgroup.

We did not specify some details about the analyses in our protocol. We chose to present sensitivity and median interquartile range values for cut-offs of specificity. 